Western University Scholarship@Western

1994

\title{
Dynamic Programming for Non-Additive Stochastic Objectives
}

Hiroyuki Ozaki

Peter A. Streufert

Follow this and additional works at: https://ir.lib.uwo.ca/economicsresrpt

Part of the Economics Commons

Citation of this paper:

Ozaki, Hiroyuki, Peter A. Streufert. "Dynamic Programming for Non-Additive Stochastic Objectives." Department of Economics Research Reports, 9416. London, ON: Department of Economics, University of Western Ontario (1994). 


\title{
RESEARCH REPORT 9416
}

\author{
Dynamic Programming for \\ Non-Add1t1ve Stochastic Object1ves \\ by \\ ECONOMICS REFEAERCE CERTRE \\ Hiroyuki Ozaki \\ and \\ MAR - 61997 \\ Peter A. Streufert \\ UHIVERSTI OF WESTERN ONTARID
}

October 1994

\author{
Department of Economics \\ Social Science Centre \\ University of Western Ontario \\ London, Ontario. CANADA
}

N6A $5 \mathrm{C2}$ 


\title{
DYNAMIC PROGRAMMING FOR NON-ADDITIVE STOCHASTIC OBJECTIVES*
}

\author{
by Hiroyuki Ozaki \\ and Peter A. Streufert ${ }^{\dagger}$ \\ University of Western Ontario \\ Department of Economics \\ Social Science Centre \\ London, Ontario N6A 5C2 \\ $519 / 661-3500$
}

October 11, 1994

\begin{abstract}
We derive the existence of an optimum and the techniques of dynamic programming for nonadditive stochastic objectives. Our key assumption for nonnegative objectives is that asymptotic impatience exceeds asymptotic "mean" growth, where "mean" growth is derived not only from intertemporal inelasticity and the random return on investment but also from the curvature of the non-additive stochastic aggregator (i.e., the "certainty equivalent"). We provide broad families of new, interesting, and tractable examples. They illustrate that "mean" growth can exist even when the distribution of returns has unbounded support, that power discounting often implies infinite asymptotic impatience, and that nonpositive objectives are easily handled with few restrictions on growth.
\end{abstract}

\footnotetext{
*Section 2 is similar to an earlier working paper with a similar title (Ozaki and Streufert, 1992). Sections 3 and 4 are entirely new.

tWe thank the Social Sciences and Humanities Research Council of Canada for financial support.
} 


\section{Introduction}

\subsection{Preamble}

We derive the existence of an optimum and the techniques of dynamic programming for non-additive stochastic objectives. Additive objectives are recursive in the sense that

$$
E_{s} \sum_{t=0}^{\infty} d^{t} u\left(c_{t}\right)=u\left(c_{0}\right)+d E_{s} \sum_{t=1}^{\infty} d^{t-1} u\left(c_{t}\right)
$$

The non-additive objectives $U_{s}(c)$ which we consider are recursive in the sense that

$$
U_{s}(c)=W\left(c_{0}, M_{s} U\left({ }_{1} c\right)\right)
$$

Here $c$ is a stochastic consumption process, the dynamic aggregator $W$ derives current utility $U_{s}(c)$ from current consumption $c_{0}$ and future "mean" utility $M_{s} U\left({ }_{1} c\right)$, and the stochastic aggregator" $M$ derives future "mean" utility from the current state $s$ (which may affect transition probabilities) and from tomorrow's utility as a function of tomorrow's state $U\left({ }_{1} c\right)$.

A critical distinction between additive and non-additive objectives is that the stochastic aggregator $M$ need not be the expectation operator $E .^{2}$ Epstein and Zin (1989), Farmer (1990), and Weil (1990) motivate such stochastic aggregators by considering cases in which $W$ is additive. In these cases, the additivity of $M$ would imply that the curvature of the felicity function $u$ determines both risk aversion and intertemporal inelasticity; the concavity of $M$ would amplify risk aversion above intertemporal inelasticity; and the convexity of $M$ would dampen risk aversion below intertemporal inelasticity. Of further interest is the fact that the concavity of $M$ would cause the agent to prefer the early resolution of uncertainty, while the convexity of $M$ would cause the agent to prefer the late resolution of uncertainty (Kreps and Porteus, 1978). Further motivation is provided by Epstein and Wang (1994a,b), who show that Knightian uncertainty can be modeled with a concave stochastic aggregator. ${ }^{3}$

The curvature of $M$ also raises a number of difficult mathematical issues which this paper resolves. Such issues have not been addressed elsewhere: Section 3.2 notes that no other literature develops the techniques of dynamic programming when $M$ is non-additive and the feasible set contains consumption processes which grow without bound over time.

One of these mathematical issues is upper convergence. When both $W$ and $M$ are additive, one needs assumptions on preferences and technology which ensure that no feasible consumption process generates infinite utility (e.g., Mehra and Prescott, 1985). When either $W$ or $M$ is

\footnotetext{
${ }^{1}$ By a stochastic aggregator we mean a "certainty equivalent" that need not satisfy certainty equivalence.

${ }^{2}$ Our theory also covers interesting objectives in which $M$ is additive and $W$ is non-additive. Such examples include the class of preferences developed by Uzawa (1968) and Epstein (1983) as well as the special cases of our examples below in which $a=1$. The model of Bhattacharya and Majumdar (1989) is broadly similar.

${ }^{3}$ Still further motivation could be drawn from the large non-expected-utility literature in atemporal models.
} 
non-additive, this finiteness must be generalized to the topological concept of upper convergence (Streufert, 1990). Upper convergence requires that replacing far future consumption levels with the highest conceivable consumption levels has asymptotically no effect. Like finiteness in the additive case, upper convergence requires that impatience asymptotically dominates the growth of "mean" felicity over time. However, this "mean" cannot be defined by mathematical expectation: when $M$ is non-additive the growth of the mathematical expectation of felicity may be completely irrelevant to upper convergence. Rather, the definition of "mean" must be based upon the stochastic aggregator $M$.

This new definition is developed in Section 3.1 and then applied to the examples of Sections 3.3 and 3.4. The examples of Section 3.3 demonstrate that increases in the concavity of $M$ lead to decreases in the mean growth factor. Hence, increases in the concavity of $M$ promote upper convergence. These new observations resemble the familiar fact that increases in the concavity of the felicity function lead to decreases in the growth of felicity and thereby promote upper convergence. However, the situation here is considerably more subtle because the concavity of $M$ is compounded with itself over time.

Of course, one could circumvent this entire discussion of mean growth by bounding growth across states with a maximum growth factor. We do this in Proposition 2 of Section 3.2, and show that this easily subsumes the current literature. But this is, at best, a "quick and dirty" solution. In many technologies, such as the lognormal and gamma examples of Sections 3.3 and 3.4, the distribution of returns is unbounded and consequently no (finite) maximum growth factor exists. Furthermore, even if the maximum growth factor does exist, it will typically be much greater than our mean growth factor, as illustrated by the loguniform technology of Section 3.3. Hence sufficient conditions for upper convergence which use a maximum growth factor are often inapplicable, and even when they do apply, they are typically much more restrictive than conditions which use our mean growth factor.

\subsection{General Theory}

Section 2 derives the existence of an optimum and the techniques of dynamic programming from abstract assumptions upon a primitive utility function $U$ and primitive aggregators $W$ and $M$.

Given upper convergence, Theorem A derives the existence of the true value function, shows that it is upper semi-continuous, characterizes it as the greatest admissible solution to Bellman's equation, and proves that its associated policy correspondence leads to optimality. Theorem B assumes not only upper convergence but also lower convergence, which is a topological axiom requiring that replacing far future consumption levels with zero consumption levels has asymptotically no effect. Theorem B augments Theorem A's conclusions by characterizing the true value function as 
the unique admissible solution to Bellman's equation. Finally, Theorem $\mathrm{C}$ adds a few additional assumptions and derives the continuity of the true value function and the upper semi-continuity of its associated policy correspondence.

This abstract theory is rather elegant. All its assumptions and conclusions are invariant to continuous monotonic transformations of the utility space.

\subsection{Nonnegative Preferences}

Section 3 constructs a nonnegative-valued utility function $U$ from primitive aggregators $W$ and $M$. Theorem D of Section 3.1 develops assumptions for $W$ and $M$ which are sufficient to imply that $U$ satisfies the abstract assumptions of Section 2's general theory. Among these assumptions is one (condition N9) which defines asymptotic mean growth and requires that asymptotic impatience exceeds asymptotic mean growth. Section 3.2 develops much more restrictive assumptions which employ a maximum growth factor, and carefully discusses how this more restrictive framework easily subsumes and extends the literature.

These results have already been introduced in Section 1.1. However, three further aspects should be highlighted. First, we use an asymptotic rather than uniform lower bound on the impatience implicit in $W$. This asymptotic bound $\delta^{-1}$ is no less than, and often much more than, the uniform bound $\bar{\delta}^{-1}$ (in fact, $\delta^{-1}=+\infty$ in Section 3.4's examples). Since a contraction mapping uses the uniform bound $\bar{\delta}^{-1}$, it is inherently less general (the same point is made by Streufert, 1990 , in a deterministic context).

Second, Theorem D allows one to define mean growth in a variety of ways. Each different way corresponds to a different approximation $N$ which bounds the stochastic aggregator $M$ and satisfies additional regularity properties. Often, one can set $N$ equal to $M$, as we do in this paper's examples. Alternatively, one could set $N$ equal to a Choquet integral, as we do in Ozaki and Streufert (1993). A third alternative is to set $N$ equal to a multiple of the supremum operator, as we do in Section 3.2 to derive our corollary for bounded growth factors. This flexibility clearly enhances Theorem D's generality.

Third, our sufficient conditions for upper convergence account not only for consumption growth due to the random return on investment but also for consumption growth due to the accumulation of random endowments. Just as we allow for unbounded support in the distribution of returns, we allow for unbounded support in the distribution of endowments.

Sections 3.3 and 3.4 study two parametric families of preferences together with three parametric families of technologies. All three families of technologies admit feasible consumption processes that grow without bound. The first family of preferences (Section 3.3) has an additive dynamic aggregator $W$ and coincides with a subset of the isoelastic preferences defined by Epstein and Zin (1989) and Weil (1990). We use these examples to illustrate the advantages of our mean 
growth factor vis-à-vis the maximum growth factor and to show that this mean growth factor falls with the parameter governing the curvature of $M$. The second family of preferences (Section 3.4) extends these examples by introducing a non-additive dynamic aggregator in which future utility is raised to an exponent less than unity. These examples of "power discounting" are entirely new in a stochastic setting and are of interest to economists because impatience increases with future utility. Further, since impatience is asymptotically infinite throughout this family, Theorem D's assumption about impatience dominating growth is automatically satisfied for any of the three families of technologies without any parametric restrictions.

\subsection{Nonpositive Preferences}

Section 3 constructed nonnegative-valued utility functions. It generalized Blackwell's (1965) theory of discounted dynamic programming, which had been designed for an additive objective whose felicity function $u$ is bounded from below (e.g., an isoelastic felicity function which is less concave than the logarithm). Lower convergence is satisfied throughout this entire class, and, as we have seen, upper convergence needs to be derived from an assumption requiring that impatience dominates growth.

In contrast, Section 4 constructs nonpositive-valued utility functions. It generalizes Strauch's (1966) theory of negative dynamic programming, which was designed for an additive objective whose felicity function $u$ is bounded from above (e.g., an isoelastic felicity function which is more concave than the logarithm). Here lower convergence is typically violated: zero consumption typically matters even in very distant time periods. As a consequence, Theorems B and C of Section 2's general theory are typically inapplicable.

On the other hand, Theorem $\mathrm{E}$ of Section 4.1 shows that upper convergence is satisfied throughout the entire class of nonpositive preferences. In particular, there needs to be no assumption linking impatience with growth. Thus the relatively limited conclusions of Theorem A are at our disposal with virtually no cost.

Sections 4.2 and 4.3 study two parametric families of preferences together with the three parametric families of technologies that were introduced in the examples of Section 3. The first family of preferences (Section 4.2) has an additive dynamic aggregator $W$ and coincides with a subset of the isoelastic preferences defined by Epstein and Zin (1989) and Weil (1990). We show that Theorem A can be applied to these examples without any parametric restrictions on the technologies, or on the curvature of the stochastic aggregator $M$, or on the discount factor (upcounting is permitted). The second family of preferences (Section 4.3) extends these examples by introducing a non-additive dynamic aggregator $W$ in which future utility is raised to an exponent other than unity. These examples of "power discouting" are entirely new to nonpositive preferences. We find that Theorem A can be applied without any parametric restrictions on the exponent by 
which $W$ discounts (or upcounts) future utility. This tractable parametric family is of interest to economists because certain exponents specify that impatience increases with future utility while other exponents specify that impatience decreases with future utility.

\subsection{Technical Issues}

The text discusses a number of other technical difficulties which were resolved in order to accommodate the non-additive aggregator $M$. Three deserve brief mention here. In Section 2.1 (and Appendix C), we develop a new concept called "upper quasi-continuity" for the non-additive stochastic aggregator $M$ : this monotonically invariant concept replaces the "Feller condition" commonly imposed upon the stochastic kernel of the expectation operator $E$. In Section 2.2 (and Appendix A), we derive the existence of an optimum by a recursive argument: as a consequence we need not topologize the space of consumption processes, nor derive the upper semi-continuity of the utility function, nor demonstrate the compactness of the feasible set. In Section 3.1, we derive something like a "spectral radius" from the nonlinear and unbounded operator combining $M$ with the growth factors: this "spectral radius" becomes the measure of mean growth discussed earlier in Section 1.1.

All lemmas and all but one proof are relegated to Appendices A through E. The lemmas and proofs are numbered consecutively from Lemma A.1 through Lemma E.3.

\section{General Theory}

\subsection{Main Assumptions}

Let $S$ be a nonempty Borel-measurable subset of a Polish space (that is, a Borel-measurable subset of a topological space which is homeomorphic to a complete separable metric space). For example, $S$ could be finite, or $S$ could be countably infinite and discrete, or $S$ could be any Borelmeasurable subset of the real line. We regard the elements $s \in S$ as single-period states.

Let $A$ be a nonempty and finite set of assets, and let $x \in \Re_{+}^{A}$ denote a portfolio of investments in these assets. Let $F: S \times \Re_{+}^{A} \rightarrow \Re_{+}$satisfy

\section{F1. $\quad F$ is upper semi-continuous .}

We regard $F_{s}\left(x_{-}\right)$as today's income, given that today's state is $s$ and that yesterday's investment portfolio was $x_{-}$. For example, $F$ could be $\Sigma_{a} F^{a}$ when each asset $a$ has its own production function $F^{a}: S \times \Re_{+} \rightarrow \Re_{+}$.

Let $L(X, Y)$ denote the space of Borel-measurable functions from one topological space $X$ into another topological space $Y$. Let $t \in\{0,1,2, \ldots\}$ denote time. A consumption process $c$ 
is an element of $\Pi_{t \geq 0} L\left(S^{t}, \Re_{+}\right)$, where $L\left(S^{0}, \Re_{+}\right) \equiv \Re_{+}$. Similarly, a portfolio process $\boldsymbol{x}$ is an element of $\Pi_{t \geq 0} L\left(S^{t}, \Re_{+}^{A}\right)$, where $L\left(S^{0}, \Re_{+}^{A}\right) \equiv \Re_{+}^{A}$. A consumption process $c$ is feasible from an initial income $y$ if there exists a corresponding portfolio process $x$ such that $c_{0}+\Sigma_{a} x_{0}^{a} \leq y$ and $(\forall t \geq 1) c_{t}+\Sigma_{a} x_{t}^{a} \leq F\left(x_{t-1}\right)$.

From the function $F$ derive the function $\bar{F}: S \times \Re_{+} \rightarrow \Re_{+}$by

$$
\bar{F}_{s}\left(y_{-}\right)=\max \left\{F_{s}\left(x_{-}\right) \mid \Sigma_{a} x_{-}^{a} \leq y_{-}\right\}
$$

The existence and upper semi-continuity of $\bar{F}$ is insured by F1 and the Maximum Theorem (Berge, 1962, p. 116, Theorem 2). The quantity $\bar{F}_{s}\left(y_{-}\right)$bounds today's income as a function of yesterday's income $y_{-}$and today's state $s$. It allows for the possibility that yesterday's consumption was zero and for the possibility that yesterday's portfolio was fortuitously chosen as if in anticipation of today's state. Repeated applications of this observation (Lemma A.1) show that if a consumption process $c$ is feasible from the initial income $y$, then $(\forall t) c_{t} \leq \bar{F}^{t}(y)$, where $\bar{F}^{t}$ is the $t$-fold composition $\bar{F} \circ \bar{F} \circ \ldots \circ \bar{F}$ (let $\bar{F}^{0}(y)=y$ ). Hence every consumption process which is feasible from $y$ satisfies $c \leq{ }_{0} \bar{F}(y)$, where ${ }_{0} \bar{F}(y)$ denotes the "pure-accumulation" process $\left\langle\bar{F}^{t}(y)\right\rangle_{t \geq 0}$. Therefore, every feasible consumption process lies in

$$
C \equiv\left\{c \in \Pi_{t} L\left(S^{t}, \Re_{+}\right) \mid(\exists y) c \leq{ }_{0} \bar{F}(y)\right\}
$$

Because of this observation, we can employ $C$ as the domain of the utility function. ${ }^{4}$ (Conveniently, $(\forall y)_{0} \bar{F}(y) \in C$ by Lemma A.1.)

Preferences are specified by a utility function $U: S \times C \rightarrow \bar{\Re}$ (where $\bar{\Re}=[-\infty, \infty]$ ). Theorem A will assume that $U$ is monotonic:

$$
\text { U1. }(\forall s)\left(\forall c^{\prime} \geq c\right) \quad U_{s}\left(c^{\prime}\right) \geq U_{s}(c)
$$

and upper convergent:

$$
\text { U2. }(\forall s)(\forall y)\left(\forall c \leq{ }_{0} \bar{F}(y)\right) \quad \lim _{t \rightarrow \infty} U_{s}\left({ }_{0} c_{t-1},{ }_{t} \bar{F}(y)\right)=U_{s}(c)
$$

where ${ }_{0} c_{t-1}$ denotes the restriction of the process $c$ to periods $\{0,1, \ldots, t-1\}$ and ${ }_{t} \bar{F}(y)$ denotes the restriction of the pure-accumulation process ${ }_{0} \bar{F}(y)$ to periods $\{t, t+1, \ldots\}$. Note that the utility sequence defining upper convergence is weakly decreasing (by $c \leq 0 \bar{F}(y)$ and U1) and bounded from below by $U_{s}(c)$. Thus the limit must exist, and the only issue is whether the sequence falls

\footnotetext{
${ }^{4}$ It is noteworthy that we never endow our function spaces with topologies or norms. In particular, we never topologize the space of consumption processes and hence never define or derive the continuity of the utility function or the compactness of the feasible set. Rather, we derive the existence of an optimum by a recursive argument in the spirit of Maitra's (1968) dynamic programming argument. Further, in spite of this lack of topological structure, we can derive the upper semi-continuity (Theorem A) and continuity (Theorem C) of the value function.
} 
all the way down to $U_{s}(c){ }^{5}$ Economically speaking, upper convergence states that replacing far future consumption levels with the very best prospect has asymptotically no effect.

Theorem B and $\mathrm{C}$ will also assume that $U$ is lower convergent:

$$
\text { U3. }(\forall s)(\forall c) \quad \lim _{t \rightarrow \infty} U_{s}\left(0 c_{t-1},{ }_{t} 0\right)=U_{s}(c) \text {, }
$$

where ${ }_{t} 0$ is the restriction of the zero-consumption process 0 to periods $\{t, t+1, \ldots\}$. Note that the utility sequence defining lower convergence is weakly increasing (by $c \geq 0$ and U1) and bounded from above by $U_{s}(c)$. Hence the limit must exist, and the only issue is whether the sequence climbs all the way up to $U_{s}(c)$. Economically speaking, lower convergence requires that replacing far future consumption levels with the most gloomy outlook has asymptotically no effect. The term biconvergence refers to the combination of $\mathrm{U} 2$ and $\mathrm{U} 3$.

As would be expected from the deterministic case (Streufert, 1990, 1992), the impatience of the utility function promotes both upper and lower convergence while the growth of the pure accumulation process hinders upper convergence. In addition, the utility function may be concave in the sense of either intertemporal inelasticity or risk aversion. Concavity in either sense promotes upper convergence and hinders lower convergence. These general remarks are substantiated by the sufficient conditions for upper and lower convergence which we develop and employ in the theorems and examples of Sections 3 and 4 (in particular, see Section 3.3).

In order to enable the recursive techniques of dynamic programming, we assume the existence of a utility space ${ }^{6} T \subset \bar{\Re}$, a dynamic aggregator $W: \Re_{+} \times T \rightarrow T$, and a stochastic aggregator $M: S \times L(S, T) \rightarrow T$ which satisfy

$$
\begin{aligned}
& \text { U4. }(\forall s)(\forall c) U_{s}(c)=W\left(c_{0}, M_{s} U\left({ }_{1} c\right)\right), \\
& \text { U5. } W \text { is weakly increasing in its second argument, } \\
& \text { U6. } W \text { is u.s.c. (in both arguments together), and } \\
& \text { U7. }(\forall s)\left(\forall u^{\prime} \geq u\right) M_{s}\left(u^{\prime}\right) \geq M_{s}(u) .
\end{aligned}
$$

Assumption U4 is a stochastic version of Koopmans' (1960) equation. ${ }^{7}$ It appeared in the first paragraph of Section 1.1.

\footnotetext{
${ }^{5}$ Upper convergence is equivalent to $(\forall y) U$ is upper semi-continuous over the product $\Pi_{t \geq 0}\left[0, \bar{F}^{t}(y)\right]$ when each coordinate space $\left[0, \vec{F}^{t}(y)\right]$ is endowed with the discrete topology. In the same fashion, lower convergence is linked to discrete-product lower semi-continuity.

${ }^{6}$ The range of $W$ (utility) could be entirely distinct from the range of $M$ ("mean" utility). Further, the domain of $M$ need only contain measurable utility-valued functions of $S$ that are within the rectangle $\left[J^{-}(y), J^{+}(y)\right]$ for some $y\left(J^{-}\right.$and $J^{+}$are defined below); and the domain of $W$ need only contain the "mean" utilities in the range of $M$. This extra generality is not worth its notational burden.

${ }^{7}$ When $U$ is applied to a consumption process $c$ at every history in $U_{t \geq 0} S^{t}$, it generates a utility process $u$. It is common to assume that $u$ is adapted in the sense that $u \in \Pi_{t \geq 0} L\left(S^{t}, T\right)$. This common assumption is routinely satisfied when $\mathrm{U} 4$ is met, because the domain of $M$ in every state is $L(S, T)$.
} 
F1 and $\mathrm{U} 6$ assume that $F$ and $W$ are u.s.c. Here we develop a similar assumption for $M$. The concept is subtle because the domain of $M$ contains a space of functions and because $M$ need not be an integral. We say that $M$ satisfies upper quasi-continuity (u.q.c.) beneath a function $\bar{u}$ if

$$
\left(\forall\left\langle s^{n}\right\rangle_{n=1}^{\infty} \rightarrow s^{0}\right)\left(\forall\left\langle u^{n}\right\rangle_{n=1}^{\infty} \leq \bar{u}\right) \quad \varlimsup_{n \rightarrow \infty} M_{s^{n}} u^{n} \leq M_{s^{0}} \varlimsup_{n \rightarrow \infty} u^{n} .
$$

If $S$ is finite (or discrete) and $M$ is monotonic (U7), then $L(S, T)$ is the product space $T^{S}$ and upper quasi-continuity beneath $\bar{u}$ is equivalent to upper semi-continuity over $S \times\left\{u \in T^{S} \mid u \leq \bar{u}\right\}$. In general, $L(S, T)$ is not a product space and we do not endow it with a topology. Appendix $\mathrm{C}$ develops sufficient conditions for upper quasi-continuity in the case that $M$ is the expectation operator. Within that special context, upper quasi-continuity may be regarded as a generalized "Feller property" (Stokey and Lucas, 1989, p. 220). ${ }^{8}$ These sufficient conditions, together with the invariance of upper quasi-continuity to continuous monotonic transformations (see U8 in Proof of Lemma B.1), are employed in the examples of Sections 3 and 4.

Finally, we incorporate this new concept of upper quasi-continuity into a specific assumption on $M$.

U8. ( $\left.\forall y_{-}\right) M$ is upper quasi-continuous beneath $J^{+} \circ \bar{F}\left(y_{-}\right)$,

where $J^{+}: S \times \Re_{+} \rightarrow T$ is defined by $J_{s}^{+}(y)=U_{s}\left({ }_{0} \bar{F}(y)\right)$. $J^{+}$is readily interpreted as an extremely optimistic value function since any consumption process that is feasible from $y$ is bounded from above by the pure-accumulation process ${ }_{0} \bar{F}(y)$. Note that both $J^{+}$and $\bar{F}$ are functions of $s$.

Lemma B.1 shows that all of the above assumptions are invariant to continuous monotonic transformations of the utility space $T$.

\subsection{Main Results}

Define the true value function $J^{*}: S \times \Re_{+} \rightarrow T$ by

$$
J_{s}^{*}(y)=\max \left\{U_{s}(c) \mid c \text { is feasible from } y\right\} .
$$

A consumption process $c$ is optimal from $y$ and $s$ if it is feasible from $y$ and satisfies $U_{s}(c)=J_{s}^{*}(y)$. Note that the existence of $J^{*}$ (that is, the logical coherency of optimality) remains to be proven (see Theorem A).

Define the policy correspondence $K^{*}: S \times \Re_{+} \rightarrow \Re_{+} \times \Re_{+}^{A}$ by

$$
K_{s}^{*}(y)=\arg \max \left\{W\left(c, M_{s} J^{*} \circ F(x)\right) \mid c+\Sigma_{a} x^{a} \leq y\right\} .
$$

\footnotetext{
${ }^{8}$ The reference to Stokey and Lucas' condition is imprecise because the domain of our $M$ is considerably more complex: it consists of measurable (rather than continuous) functions which need not be bounded and which need not be defined over a compact state space.
} 
A consumption process $c$ is recursively optimal from $y$ and $s$ if there exists a corresponding portfolio process $x$ such that $\left(c_{0}, x_{0}\right) \in K_{s}^{*}(y)$ and $(\forall t \geq 1)\left(c_{t}, x_{t}\right) \in K^{*} \circ F\left(x_{t-1}\right)$. Note that the existence of $K^{*}$ (that is, the logical coherency of recursive optimality) remains to be proven (see Theorem $\mathrm{A}$ and Lemma A.2).

A function $J: S \times \Re_{+} \rightarrow T$ is an admissible value function if it is upper semi-continuous, ${ }^{9}$ weakly increasing in its second argument, and satisfies $J^{-} \leq J \leq J^{+}$, where $J^{+}$is the extremely optimistic value function defined earlier and $J^{-}: S \times \Re_{+} \rightarrow T$ is the extremely pessimistic value function defined by $J_{s}^{-}(y)=U_{s}(\mathbf{0})$. Define Bellman's operator $B$ from the set of admissible value functions into itself by

$$
(\forall s)(\forall y) \quad B J_{s}(y)=\max \left\{W\left(c, M_{s} J \circ F(x)\right) \mid c+\Sigma_{a} x^{a} \leq y\right\}
$$

Lemma A.5 shows that $B$ is well-defined (that is, that $B J$ exists and is admissible whenever $J$ is admissible). Finally, we say that $J$ solves Bellman's equation if $J=B J$.

The following theorems provide the fundamental techniques of dynamic programming. Under upper convergence (U2), Theorem A derives the existence of $J^{*}$, derives optimality from recursive optimality, and provides a limited characterization of $J^{*}$. Under biconvergence (U2 and U3), Theorem B augments Theorem A with a complete characterization of $J^{*}$.

Theorem A: Assume F1, U1-U2, and U4-U8. Then $J^{*}$ exists, it is the greatest ${ }^{10}$ admissible solution to Bellman's equation, and it equals $\lim _{n \rightarrow \infty} B^{n} J^{+} .11$ Furthermore, recursive optimality implies optimality. (Proof A.6.)

Theorem B: Assume F1 and U1-U8. Then $J^{*}$ exists, it is the unique admissible solution to Bellman's equation, and it is equal to both $\lim _{n \rightarrow \infty} B^{n} J^{+}$and $\lim _{n \rightarrow \infty} B^{n} J^{-}$. Furthermore, recursive optimality implies optimality. (Proof A.7.)

\subsection{Corollary Giving Topological Properties for $J^{*}$ and $K^{*}$}

Theorem $\mathrm{C}$ adds the following assumptions.

F2. $\quad F$ is lower semi-continuous,

U9. W is lower semi-continuous, and

U10. $M$ is lower quasi-continuous above $J^{-}(0)$,

\footnotetext{
${ }^{9}$ Since upper semi-continuity implies the existence of a measurable selection (Lemma A.2), we need not explicitly assume the existence of a measurable selection within our concept of admissibility.

${ }^{10}$ That is, $J^{*}$ is an admissible solution to Bellman's equation and $(\forall s)(\forall y) J_{0}^{\prime}(y) \leq J_{\bullet}^{*}(y)$ for any other admissible solution $J^{\prime}$.

${ }^{13}$ The limit is pointwise.
} 
where $M$ is lower quasi-continuous (l.q.c.) above a function $\underline{u}$ if

$$
\left(\forall\left\langle s^{n}\right\rangle_{n=1}^{\infty} \rightarrow s^{0}\right)\left(\forall\left\langle u^{n}\right\rangle_{n=1}^{\infty} \geq \underline{u}\right) \quad \underline{\lim }_{n \rightarrow \infty} M_{s^{n}} u^{n} \geq M_{s^{0}} \underline{\varliminf}_{n \rightarrow \infty} u^{n}
$$

As all previous assumptions, these additional assumptions are invariant to continuous monotonic transformations of the utility space $T$ (Lemma B.1). Lower quasi-continuity resembles the concept of upper quasi-continuity discussed before U8. Lemma C.3 provides a sufficient condition for lower quasi-continuity which is used in the examples of Section 3.

Theorem C: Assume F1-F2 and U1-U10. Then $J^{*}$ is continuous and $K^{*}$ is upper semicontinuous $^{12}$. (Proof A.8.)

\section{Nonnegative Objectives}

This section constructs a nonnegative-valued utility function $U$ from a dynamic aggregator $W$ and a stochastic aggregator $M$. Section 3.1 develops assumptions for $W ; M$, and the production function $F$ which are sufficient to imply assumptions U1-U8 of Section 2's general theory. Section 3.2 derives an easily applied corollary for a very special case that easily subsumes the current literature. Sections 3.3 and 3.4 exhibit broad classes of interesting examples which illustrate the full force of our theory.

\subsection{Sufficient Conditions}

Suppose that a dynamic aggregator $W: \Re_{+} \times \bar{\Re}_{+} \rightarrow \bar{\Re}_{+}$and a stochastic aggregator $M: S \times L\left(S, \bar{\Re}_{+}\right) \rightarrow \bar{\Re}_{+}$satisfy $^{13}$

A1. $W$ is weakly increasing ,

A2. $W$ is u.s.c.,

A3. $W$ is continuous in its second argument,

A4. $(\forall s)\left(\forall u^{\prime} \geq u\right) \quad M_{s}\left(u^{\prime}\right) \geq M_{s}(u)$,

A5. $(\forall s)\left(\forall\right.$ weakly increasing $\left.\left\langle u^{n}\right\rangle_{n=1}^{\infty}\right) \quad \lim _{n \rightarrow \infty} M_{s} u^{n}=M_{s} \lim _{n \rightarrow \infty} u^{n}$, and

A6. $(\forall t)\left(\forall u_{t+1} \in L\left(S^{t+1}, \bar{\Re}_{+}\right)\right)$the $\operatorname{map}_{1} s_{t} \mapsto M_{s_{t}} u_{t+1}\left(s_{1}, \cdot\right)$ is in $L\left(S^{t}, \bar{\Re}_{+}\right)$.

\footnotetext{
${ }^{12}$ We employ Berge's (1963, p. 109) concept of upper semi-continuity, which includes compact-valuedness.

${ }^{13}$ The utility number $+\infty$ simplifies the definition of $M$ and $N$ (their domains are simply collections of measurable functions rather than collections of measurable functions whose image is finite), and thereby simplifies the adaptedness conditions A6 and N3. Note that Theorem D derives the finiteness of utility at every $c$ in $C$ (see theorem's statement) and also the finiteness of mean future utility at every history and every $c$ in $C$ (see section titled "Well-Definition and Real-Valuedness" in Proof D.1).
} 
The convergence and adaptedness conditions of A5 and A6 will be used to justify our construction of $U$ and to prove Koopmans' equation (U4). Note that the map in A6 could be suggestedly labeled $u_{t}$.

Derive from the dynamic aggregator $W$ the real numbers

$$
\begin{aligned}
\delta & =\limsup _{m \rightarrow+\infty} \sup _{c} \sup _{m^{\prime}>m} \frac{W\left(c, m^{\prime}\right)-W(c, m)}{m^{\prime}-m} \text { and } \\
\bar{\delta} & =\sup _{m<+\infty} \sup _{c} \sup _{m^{\prime}>m} \frac{W\left(c, m^{\prime}\right)-W(c, m)}{m^{\prime}-m}
\end{aligned}
$$

where $m^{\prime} \in \Re_{+}$. (When $W$ is continuously differentiable over $\Re_{+}^{2}$, the expression $\sup _{m^{\prime}>m}\left[W\left(c, m^{\prime}\right)-\right.$ $W(c, m)] /\left[m^{\prime}-m\right]$ may be replaced with the derivative of $W$ with respect to its second argument.) In the familiar case $W(c, m)=c^{e}+d m$, the slope $\left[W\left(c, m^{\prime}\right)-W(c, m)\right] /\left[m^{\prime}-m\right]$ is constant at $d$ and thus $\bar{\delta}=\delta=d$. In general, this slope is called the variable discount factor, or alternatively, time perspective (Koopmans, Diamond, and Williamson, 1964). The constant $\bar{\delta}$ is a uniform (i.e., Lipschitz) upper bound on the variable discount factor (Lucas and Stokey, 1984), while $\delta$ is an asymptotic upper bound on the variable discount factor (Streufert, 1990). Note that $\delta \leq \bar{\delta}$ always, and that $\delta<\bar{\delta}$ rather frequently (for example, $\delta=0$ in all the examples of Section 3.4). Since the discount factor is inversely related to impatience, these upper bounds on time perspective correspond to lower bounds on impatience.

Consider the dynamic aggregator $W$ again, and derive an $\varepsilon \in(0,1]$ such that

$$
\left(\exists \kappa_{1}, \kappa_{2} \in \Re_{+}\right)(\forall c) \quad W(c, 0) \leq \kappa_{1}+\kappa_{2} c^{e}
$$

In the familiar case $W(c, m)=c^{e}+d m$ where $e \in(0,1]$, we can set $\varepsilon$ as low as $e$. In the happy event that $W(c, 0)$ is bounded, we can set $\varepsilon$ arbitrarily close to 0 . In general, the constant $\varepsilon$ provides an asymptotic upper bound on intertemporal elasticity (Brock and Gale, 1968). Because $\varepsilon$ may not exceed unity, the existence of $\varepsilon$ precludes a dynamic aggregator which is asymptotically convex in consumption.

Consider the production function $F$ and find two functions $\zeta$ and $\gamma$ in $L\left(S, \Re_{+}\right)$such that

$$
(\forall s)\left(\forall y_{-}\right) \quad \bar{F}_{s}\left(y_{-}\right) \leq \zeta_{s}+\gamma_{s} y_{-} .
$$

These functions will be used to generate convenient upper bounds on the pure-accumulation process ${ }_{0} \bar{F}$. Intuitively we may regard $\zeta$ as "the" random endowment and $\gamma$ as "the" growth factor. However, one has considerable flexibility in choosing convenient functions for a given problem (for example, one might aim to satisfy the assumptions of Proposition 1 below).

Finally consider the state space $S$ and the stochastic aggregator $M$. Elsewhere we consider the measurable space $S=\left(S, \mathcal{B}_{S}\right)$. Now find a $\sigma$-algebra $\mathcal{A}$ for the set $S$ which is at least as fine 
as $\mathcal{B}_{S}$, and let $\widehat{S}=(S, \mathcal{A})$ denote the resulting measurable space. Then find a (possibly nonlinear) operator $N: S \times L\left(\widehat{S}, \bar{\Re}_{+}\right) \rightarrow \bar{\Re}_{+}$such that

N1. $(\forall s)\left(\forall u^{\prime} \geq u\right) \quad N_{s}\left(u^{\prime}\right) \geq N_{s}(u)$,

N2. $(\forall s)\left(\forall\right.$ weakly increasing $\left.\left\langle u^{n}\right\rangle_{n=1}^{\infty}\right) \lim _{n \rightarrow \infty} N_{s} u^{n}=N_{s} \lim _{n \rightarrow \infty} u^{n}$,

N3. $(\forall t)\left(\forall u_{t+1} \in L\left(\widehat{S}^{t+1}, \bar{\Re}_{+}\right)\right)$the $\operatorname{map}_{1} s_{t} \mapsto N_{s_{t}} u_{t+1}\left({ }_{1} s_{t}, \cdot\right)$ is in $L\left(\widehat{S}^{t}, \bar{\Re}_{+}\right)$,

N4. $(\forall s)\left(\forall u^{\prime} \geq u\right) \quad M_{s}\left(u^{\prime}\right)-M_{s}(u) \leq N_{s}\left(u^{\prime}-u\right)$ and $M_{s}\left(u^{\prime}\right) \leq N_{s}\left(u^{\prime}\right)^{14}$

N5. $(\forall s)\left(\forall u^{\prime} \geq u\right) \quad N_{s}\left(u^{\prime}\right)-N_{s}(u) \leq N_{s}\left(u^{\prime}-u\right)$, and

N6. $(\forall t)(\forall u)(\forall \alpha>0) \quad N_{s} \alpha u \leq \alpha N_{s} u$.

In essence, assumptions N1-N6 require that $N$ is a convenient approximation to the stochastic aggregator $M$. Like $M$ (recall A4-A6), $N$ must have basic monotonicity (N1), convergence (N2), and adaptedness (N3) properties. Further, it must bound both increments to $M$ and the level of $M$ (N4). Finally, $N$ must possess two convenient properties that $M$ need not have: subadditivity (N5) and "subhomogeneity" (N6). Section 3.2 will show how making $\widehat{S}$ discrete and setting $N$ equal to a multiple of the supremum operator neatly subsumes the current literature (all the above properties except N4 are then immediate). On the other hand, Section 3.3 and 3.4 handle many new examples by setting $N=M$ and taking monotonic transformations as needed. In yet another application, Ozaki and Streufert (1993) define $N$ as a Choquet integral with capacities. This flexibility in the choice of $N$ considerably enhances the generality of Theorem D's assumptions.

The individual components discussed thus far must together satisfy

N7. $\lambda_{1} \equiv \sup _{s} N_{s} 1<\bar{\delta}^{-1}$,

N8. $(\forall s)(\forall t \geq 1) \quad g_{s}^{t} \equiv N_{s}^{t}\left(\prod_{q=1}^{t} \gamma_{s_{q}}^{\epsilon}\right)$ is finite,

N9. $\lambda_{g} \equiv \sup _{s} \varlimsup_{t \rightarrow \infty}\left(g_{s}^{t}\right)^{1 / t}<\delta^{-1}$,

N10. $(\forall s)(\forall t \geq 1) \quad z_{s}^{t} \equiv N_{s}^{t}\left(\sum_{r=1}^{t}\left(\prod_{q=r+1}^{t} \gamma_{s_{q}}^{\varepsilon}\right) \zeta_{s_{r}}^{\varepsilon}\right)$ is finite ,

N11. $\lambda_{z} \equiv \sup _{s} \varlimsup_{t \rightarrow \infty}\left(z_{s}^{t}\right)^{1 / t}<\delta^{-1}$, and

N12. $\left(\forall k_{1}, k_{2} \in \Re_{+}\right)\left(\forall d<\left(\lambda_{g} \vee \lambda_{z}\right)^{-1}\right)\left(\forall y_{-}\right)$

$M$ is upper quasi-continuous beneath $\overline{\bar{u}} \equiv k_{1}+k_{2} \sum_{t=0}^{\infty} d^{t}\left(z_{s}^{t}+g_{s}^{t}\left(\zeta_{s}^{\varepsilon}+\gamma_{s}^{\varepsilon} y_{-}^{\varepsilon}\right)\right)$,

where $N_{s}^{t}$ denotes the $t$-fold composition $N_{s} \circ N \circ \cdots \circ N$, and the empty product $\Pi_{q=t+1}^{t} \gamma_{s_{q}}^{\epsilon}$ is set

\footnotetext{
${ }^{14}$ The second inequality follows from the first if $M$ is normalized so that $M_{s} 0=0$.
} 
equal to unity. Unlike the other conditions, N7 is straightforward. It is clearly satisfied if $\bar{\delta}<1$ and $N$ satisfies certainty equivalence.

In $\mathrm{N} 8, g_{s}^{t}$ is defined as the mean accumulated growth factor given that $t$ periods have elapsed and that the initial state was $s$. While the initial state $s$ has no bearing whatsoever upon the accumulated growth factors $\Pi_{q=1}^{t} \gamma_{s_{q}}^{\varepsilon}$, it can affect the perceived mean of the accumulated growth factors by affecting the initial transition probabilities. Note that the exponent $\varepsilon$ in the definition of $g_{s}^{t}$ serves to buffer growth by intertemporal inelasticity. Also note that the existence of each $g_{s}^{t}$ in $\bar{\Re}_{+}$is guaranteed by N3, and consequently, the force of condition N8 is to rule out $g_{s}^{t}=+\infty$.

In $\mathrm{N} 9, \lambda_{g}$ is defined as the asymptotic mean growth factor. ${ }^{15} \mathrm{~N} 9$ 's inequality requires that asymptotic impatience exceeds asymptotic mean growth. This is Theorem D's key assumption. The order of $\sup _{s}$ and $\varlimsup_{t \rightarrow \infty}$ in the definition of $\lambda_{g}$ is one of the critical contributions of our work. Were the order switched, we would be unable to handle unbounded distributions of growth factors, and consequently, we would be unable to deal with the lognormal and gamma examples in Sections 3.3 and 3.4.

Conditions $\mathrm{N} 10$ and $\mathrm{N} 11$ for the random endowment $\zeta$ resemble conditions N8 and N9 for the growth factor $\gamma$. In N10, $z_{s}^{t}$ is defined as the mean accumulated random endowment given that $t$ periods have elapsed and that the initial state was $s$. As with $g_{s}^{t}$, the only role of the initial state $s$ is to affect the perceived likelihood of certain histories and hence to affect the perceived mean of the accumulated random endowment. All terms are buffered with the exponent $\varepsilon$ to account for intertemporal inelasticity, and the existence of $z_{s}^{t}$ in $\bar{\Re}_{+}$is guaranteed by N3. N11 defines $\lambda_{z}$ as the asymptotic mean growth factor due to the random endowment, and then requires that this factor is asymptotically dominated by impatience. As with $\lambda_{g}$, the order of $\sup _{s}$ and $\varlimsup_{\lim } \rightarrow \infty$ in the definition of $\lambda_{z}$ is a substantial contribution.

The preceding conditions will allow us to construct upper bounds on utility of the form used to define $\overline{\bar{u}}$ in N12. Thus N12 requires that $M$ be well-behaved over the subset of $L\left(S, \bar{\Re}_{+}\right)$ which could be conceivably encountered. Theorem D uses N12 only to derive U8.

Define $U: S \times C \rightarrow \Re_{+}$by

$$
(\forall s)(\forall c) \quad U_{s}(c)=\lim _{t \rightarrow \infty} W\left(c_{0}, M_{s} W\left(c_{1}, M \cdots W\left(c_{t}, 0\right) \cdots\right)\right) .
$$

The well-definition and real-valuedness of $U$ are among the conclusions of the following theorem.

Theorem D: Assume $F 1$ and A1-A6. Further assume that $\bar{\delta}, \delta, \varepsilon, \zeta, \gamma$, and $N$ exist and satisfy N1-N12. Then $U$ is well-defined, real-valued, and satisfies U1-U8. (Proof D.1.)

It is a indeed happy day if one can choose $\zeta=0:$ N10 and N11 are vacuously satisfied and

\footnotetext{
${ }^{15}$ If the stochastic aggregator $M$ were a linear operator and its domain were to consist of a normed function space, it would be natural to define the mean growth factor as the spectral radius of a linear operator combining $M$ and $\gamma$. However, $M$ is nonlinear, and its domain doesn't even have a topology. Moreover, the sup norm would invert the order of the sup, and $\overline{\lim }_{\mathrm{t} \rightarrow \infty}$.
} 
N12 is considerably simpler. The following proposition shows that life is just about as good if one can arrange $\gamma$ to exceed both 1 and some multiple of $\zeta$. (Sadly, the condition $\gamma \geq 1$ is difficult to relax: if $\gamma$ sometimes pays negative net interest, then no large initial endowment of the $\gamma$-asset could bound a positive nonrandom endowment at all histories.)

Proposition 1: Assume that $\gamma \geq 1$, that $(\exists k>0) \gamma \geq k \zeta$, and that N6 holds. Then N10 is implied by N8, N11 is implied by N9, and N12 is implied by $\left(\forall k_{1}, k_{2} \in \Re_{+}\right)\left(\forall d<\lambda_{g}^{-1}\right) M$ is upper quasi-continuous beneath $k_{1}+k_{2} \gamma_{s}^{\varepsilon} \sum_{t=0}^{\infty} d^{t}(t+1) g_{s}^{t}$. (Proof D.2.)

\subsection{Special Case: Bounded Returns}

We will argue that the following proposition substantially extends the existing literature, and that it is itself a very special case of Theorem D. Furthermore, the proposition's assumptions are easily understood.

Proposition 2: Assume F1, A1-A6, that $M_{s} 0=0$, and that $M$ is u.q.c. beneath any constant real-valued function. ${ }^{16}$ Further assume that $\bar{\delta}, \delta$, and $\varepsilon$ exist; that $\beta \in \Re_{+}$satisfies $(\forall s)(\forall u)\left(\forall a \in \Re_{+}\right) M_{s}(u+a)-M_{s}(u) \leq \beta a$; and that $z \in \Re_{+}$and $g \in \Re_{+} \operatorname{satisfy}(\forall s)\left(\forall y_{-}\right) \bar{F}_{s}\left(y_{-}\right) \leq$ $z+g y_{-} .{ }^{17}$ Finally assume $\beta<\bar{\delta}^{-1}$ and

$$
\beta g^{\varepsilon}<\delta^{-1}
$$

Then the assumptions of Theorems $B$ and $D$ are met.

Proof: We may assume $g \geq 1$ without loss of generality: $\beta<\delta^{-1}$ by the assumption $\beta<\bar{\delta}^{-1}$ and the fact that $\delta \leq \bar{\delta}$. Set $\zeta$ equal to the constant $z$, set $\gamma$ equal to the constant $g$, make the $\sigma$-algebra for $\widehat{S}$ discrete, and set $N_{s}(u)=\beta \sup _{s_{+}} u_{s_{+}} . \mathrm{N} 1, \mathrm{~N} 2, \mathrm{~N} 3, \mathrm{~N} 5$, and N6 are then immediate. The first inequality of $\mathrm{N} 4$ follows from the definition of $\beta:(\forall s)\left(\forall u^{\prime} \geq u\right) M_{s}\left(u^{\prime}\right)-$ $M_{s}(u) \leq M_{s}\left(u+\sup _{s_{+}}\left(u_{s_{+}}^{\prime}-u_{s_{+}}\right)\right)-M_{s}(u) \leq \beta \sup _{s_{+}}\left(u_{s_{+}}^{\prime}-u_{s_{+}}\right)=N_{s}\left(u^{\prime}-u\right)$ (this holds even if $\left.\sup _{s_{+}}\left(u_{s_{+}}^{\prime}-u_{s_{+}}\right)=+\infty\right)$; and the second inequality holds by the first inequality and the assumption $M_{s} 0=0 . \quad \mathrm{N} 7$ follows from the assumption $\beta<\bar{\delta}^{-1}$ because $\sup _{s} N_{s} 1=\beta$. N8 is immediate: $(\forall t)(\forall s) g_{s}^{t}=\beta^{t} \sup _{1 s_{t}}\left(\prod_{q=1}^{t} \gamma_{s_{q}}^{\varepsilon}\right)=\beta^{t}\left(g^{\varepsilon}\right)^{t}<+\infty$. N9 follows from the preceding equalities and the assumption $\beta g^{\varepsilon}<\delta^{-1}$ because $\lambda_{g}=\beta g^{\varepsilon}$. The assumptions of Proposition 1 are met by the vacuous assumption $g \geq 1$ made at the start of this proof and by the existence of $z \in \Re_{+}$: set

\footnotetext{
${ }^{16} M$ is u.q.c. beneath all constant real-valued functions whenever $M$ is a continuous monotonic transformation of an expectation operator with a strongly continuous stochastic kernel (Lemmas B.1 and C.2). This sufficient condition is satisfied by all stochastic aggregators of the form $M_{s}=\left(E_{s}(\cdot)^{\alpha}\right)^{1 / a}$. Also note that assumption $M V .1(b)$ of Epstein and Zin (1989) resembles upper quasi-continuity beneath all constant real-valued functions. A formal comparison is impossible because they formulate dynamic stochastic consumption with distributions rather than stochastic processes.

${ }^{17}$ Equivalently, assume the existence of $\zeta$ and $\gamma$ such that $z \equiv \sup _{s} \zeta_{s}<+\infty$ and $g \equiv \sup _{s} \gamma_{0}<+\infty$.
} 
$k=1 / z$. Hence $\mathrm{N} 10$ is implied by N8, N11 is implied by N9, and N12 is implied by the assumption that $M$ is u.q.c. beneath any constant: $\left(\forall k_{1}, k_{2} \in \Re_{+}\right)\left(\forall d<\lambda_{g}^{-1}\right) k_{1}+k_{2} \gamma_{s}^{\varepsilon} \sum_{t=0}^{\infty} d^{t}(t+1) g_{s}^{t}$ equals the constant $k_{1}+k_{2} g^{\varepsilon} \sum_{t=0}^{\infty} d^{t}(t+1) \beta^{t}\left(g^{\varepsilon}\right)^{t}=k_{1}+k_{2} g^{\varepsilon} \sum_{t=0}^{\infty}(t+1)\left(d \lambda_{g}\right)^{t}=k_{1}+k_{2} g^{\varepsilon}\left(1-d \lambda_{g}\right)^{-2}$.

To our knowledge, there is no work elsewhere in the literature which derives the techniques of dynamic programming when the stochastic aggregator is not the expectation operator and when there are feasible streams which grow without bound over time. ${ }^{18}$ Further, only Epstein and Zin (1989) derive the existence of an optimum against exogenous constraints, and this is a special parametric case of Proposition $2 .{ }^{19}$ Hence Proposition 2 is in itself a substantial contribution.

Furthermore, we believe that Proposition 2 could not be derived using a contraction mapping. We say this because if the present model were specialized to a deterministic context, Boyd's (1990) weighted contraction mapping technique would require the inequality

$$
\beta g^{\varepsilon}<\bar{\delta}^{-1}
$$

This inequality is considerably less general than (2) because the asymptotic measure of impatience $\delta^{-1}$ in (2) is at least as big as the uniform measure of impatience $\bar{\delta}^{-1}$. This increased generality is illustrated well by Section 3.4's examples of power discounting. In those cases, $\delta^{-1}=+\infty$ and (2) is vacuously satisfied.

Proposition 2 depends upon the existence of the maximum growth factor $g \equiv \sup _{s} \gamma_{s} \in \Re_{+}$. The work of Epstein and Zin (1989) and Epstein and Wang (1994a,b) similarly relies upon the existence of a maximum growth factor. ${ }^{20}$

Theorem $\mathrm{D}$ is much more general than Proposition 2 because it uses a mean growth factor $\lambda_{g} \in \Re_{+}$rather than the maximum growth factor $g \equiv \sup _{s} \gamma_{s} \in \Re_{+}$. For many technologies, Proposition 2 is inapplicable because the maximum growth factor $\sup _{s} \gamma_{s}$ does not exist (i.e., is

\footnotetext{
${ }^{18}$ Schäl (1978) and Bertsekas and Shreve (1978, Chapters 5 and 6) contain axiomatic theories of dynamic programming within the mathematics literature. These theories specify their models in a very different fashion, provide nothing comparable to the sufficient conditions or examples of this section, and do not discuss fundamental economic issues such as long-run growth, asymptotic discounting, intertemporal inelasticity, risk aversion, and the curvature of $M$.

${ }^{19}$ Epstein and Zin (1989) assume that a monotonic transformation of the dynamic aggregator is additive and CES, and that the production function is linear with no endowment and a random but bounded rate of return (in contrast, Proposition 2 admits general dynamic aggregators, nonconcave production functions, and random endowments). They then derive existence for utility functions that can be defined via (1) of this section. In this case they impose the inequality (3) discussed in the following paragraph. They also derive existence for utility functions that can be defined via (4) of Section 4. This is a special case of Theorem E. (In addition, they derive existence for a third class of utility functions that are defined in a still different fashion but nonetheless fall within the general theory of Section 2. This third class corresponds to relaxing the restriction $a>0$ in Utility Example 1 below).

${ }^{20}$ Epstein and Wang (1994b) develop a sophisticated theory of recursive utility but do not derive the existence of an optimum against exogenous constraints nor the techniques of dynamic programming. Rather they use a contraction mapping to derive the existence of a real-valued utility function which solves Koopmans' equation. Then they characterize, and derive the existence of, asset prices which would induce an economic agent to consume her random endowment rather than participating in the asset market.
} 
infinite), and yet, Theorem $\mathrm{D}$ can be applied because a suitable mean $\lambda_{g}$ can be found. For example, in the lognormal and gamma technologies of Sections 3.3 and 3.4, Proposition 2 is inapplicable because $\sup _{s} \gamma_{s}$ does not exist, and yet, Theorem D can be applied because suitable $\lambda_{g}$ 's can be found (see the left-hand sides of Proposition 3's inequalities).

Even in instances where the maximum sup $\gamma_{s}$ exists, one can usually find a mean $\lambda_{g}$ which is less. This can significantly increase the range of parameter values that can be accommodated (compare N9 with (2)). For example, in the loguniform technology of Section 3.3, Theorem D can be applied over a much broader range of parameters than Proposition 2 (see the discussion following Proposition 3).

Two further aspects of Theorem D's increased generality bear emphasis. First, Theorem $\mathrm{D}$ uses the mean $\lambda_{z}$ rather than the maximum $\sup _{s} \zeta_{s}$ of Proposition 2 to measure the random endowment's contribution to growth. Hence only Theorem D can be applied when $\sup _{s} \zeta_{s}$ does not exist. Further, even when $\sup _{s} \zeta_{s}$ does exist, Theorem $\mathrm{D}$ will be applicable over a broader range of parameter values. Second, Theorem $\mathrm{D}$ admits a variety of different approximations $N$, and thus admits considerable flexibility in the selection of mean $\lambda_{g}$ and $\lambda_{z} \cdot{ }^{21}$ Two interesting sorts of approximation $N$ have been studied thus far: the examples of Sections 3.3 and 3.4 use $M$ itself, and Ozaki and Streufert (1993) use Choquet integrals defined with capacities.

\subsection{Generalized Isoelastic Preferences}

Utility Example 1: Define $W: \Re_{+} \times \bar{\Re}_{+} \rightarrow \bar{\Re}_{+}$by $W(c, m)=c^{e}+d m$ where $e \in(0,1]$ and $d \in(0,1)$. Define $M: S \times L\left(S, \bar{\Re}_{+}\right) \rightarrow \bar{\Re}_{+}$by $M_{s} u=\left(E_{s} u^{a}\right)^{1 / a}$ where $a>0$. Define $U$ by (1).

The reward function $c^{e}$ is linear when $e=1$ and increasingly concave as $e$ falls toward 0 (greater concavity and thereby greater intertemporal inelasticity is accommodated in the nonpositive utility examples of Section 4). When $a=1$, the stochastic aggregator $M$ is linear, the utility function $U$ is time-additive and expected-utility, and risk aversion is identical to intertemporal inelasticity. When $a \in(1,+\infty), M$ is convex and risk aversion is less than intertemporal inelasticity. Conversely when $a \in(0,1), M$ is moderately concave and risk aversion exceeds intertemporal inelasticity (the greater concavity of $a \leq 0$ causes subtle difficulties as the finite-state-space examples of Streufert, 1991, demonstrate). Such preferences were originally defined by Epstein and Zin (1989) and Weil (1990) in an infinite-horizon model (Farmer's (1990) finite-horizon RINCE preferences are recovered by setting $a=1 / e)$.

Technology Example 1 (Lognormal Returns): Let $S=\Re$, and suppose that tomorrow's state is distributed normally with mean $b s$ and variance $\sigma^{2}$, where $s$ is today's state, $b \in(-1,1)$,

\footnotetext{
${ }^{21} N$ can even be made a multiple of the supremum operator so that the "mean" is in fact the maximum. This is how Proposition 2 is a corollary of Theorem D.
} 
and $\sigma^{2} \in \Re_{++}$. Let there be one asset, and let $F_{s}(x)=\exp (s) x$.

Technology Example 2 (Loguniform Returns): Let $S=\left[-r(1-b)^{-1}, r(1-b)^{-1}\right]$ and suppose that tomorrow's state is distributed uniformly over the interval $[b s-r, b s+r]$, where $s$ is today's state, $b \in(-1,1)$, and $r \in \Re_{++}$. Let there be one asset and define $F_{s}(x)=\exp (s) x$.

Technology Example 3 (Gamma Returns): Let $S=\Re_{++}$, and suppose that tomorrow's state has a gamma distribution with mean $r s^{b}$ and degree $r$ (i.e., the $\lambda$-parameter is $s^{-b}$ ), where $b \in(-1,1)$ and $r \in \Re_{++}$. Let there be one asset and define $F_{s}(x)=s x$.

Proposition 3: $U$ is real-valued and the assumptions of Theorems $B$ and $C$ are met, given Utility Example 1 together with any of the following:

Technology Example 1 (Lognormal) satisfying

$$
\exp \left(\frac{a}{2}\left(\frac{e \sigma}{1-b}\right)^{2}\right)<d^{-1}
$$

Technology Example 2 (Loguniform) satisfying

$$
\left(\frac{\exp (k)-\exp (-k)}{2 k}\right)^{1 / a}<d^{-1}, \text { where } k=\frac{a e r}{1-b},
$$

or Technology Example 3 (Gamma) satisfying

$$
\left(\frac{\Gamma\left(r+\frac{a e}{1-b}\right)}{\Gamma(r)}\right)^{1 / a}<d^{-1}
$$

It is clearly best to define $\gamma$ by $(\forall s) \gamma_{s}=\exp (s)$ in the lognormal and loguniform cases, and by $(\forall s) \gamma_{s}=s$ in the gamma case. In each case, the LHS of the above inequality should be understood as the mean growth factor $\lambda_{g}$ when the approximation $N$ is set equal to the stochastic aggregator $M$, and hence the entire inequality should be understood as $\mathrm{N} 9$, the key assumption of Theorem D. ${ }^{22}$ In the lognormal and gamma cases, this mean growth factor is finite even though the maximum growth factor $\sup _{s} \gamma_{s}^{\varepsilon}$ is infinite. Hence Theorem D works where Proposition 2 fails. In the loguniform case, $\sup _{s} \gamma_{s}=\exp \left(r(1-b)^{-1}\right)$ is finite and hence Proposition 2 is applicable when $\exp \left(\operatorname{er}(1-b)^{-1}\right)<d^{-1} .^{23}$ However, several lines of algebra demonstrate that this maximum growth factor is always greater than Proposition 3's mean growth factor. For example, when $a=e=r=1$

\footnotetext{
${ }^{22}$ When $a \geq 1$, the LHS is literally $\lambda_{g}$ and $N$ is literally $M$. When $a \in(0,1)$, the proposition is proven via monotonic transformations and these statements stand in a heuristic sense only.

${ }^{23}$ When $a \geq 1$, set $\bar{\delta}=\delta=d, \epsilon=e, \beta=1, z=0$, and $g=\exp \left(r(1-b)^{-1}\right)$. When $a \in(0,1)$, consider the monotonic transformation $\varphi(\cdot)=(\cdot)^{a}$ and the resulting aggregator $\widehat{W}(c, \widehat{m})=\left(c^{e}+d \widehat{m}^{1 / a}\right)^{a}$ and $\widehat{M}_{s}$ equal to the expectation operator. Then apply Proposition 2 with $\bar{\delta}=\delta=d^{a}, \varepsilon=a e, \beta=1, z=0$, and $g=\exp \left(r(1-b)^{-1}\right)$. (2) is then $d^{a} \exp \left(a e r(1-b)^{-1}\right)<1$ which is equivalent to $\exp \left(\operatorname{er}(1-b)^{-1}\right)<d^{-1}$.
} 
and $b=1 / 2$, the maximum growth factor $\exp \left(\operatorname{er}(1-b)^{-1}\right)$ is $\exp (2)$ and the mean growth factor $([\exp (k)-\exp (-k)] / 2 k)^{1 / a}$ is $[\exp (2)-\exp (-2)] / 4$.

These formulas reveal how the curvature of the stochastic aggregator tends to affect the mean growth factor. In all three technologies, the mean growth factor increases with the parameter $a$ (don't try to verify this fact for the loguniform or gamma technologies without the aid of a computer!). Hence the concavity of the stochastic aggregator tends to depress the mean growth factor and thereby promote upper convergence. Intuitively, increases in concavity increase risk aversion and thereby decrease the utility gained from stochastic growth.

Similarly, the effect of $e$ in the above formulas reveals that the concavity of the felicity function also tends to depress the mean growth factor and thereby promote upper convergence. Intuitively, increases in the concavity of the felicity function increase both risk aversion and intertemporal inelasticity and thereby decrease the utility gained from stochastic growth via two different channels. Hence there is some intuition behind the fact that $e$ has a more pronounced effect than $a$ in the above formulas.

\subsection{Power Discounting}

Utility Example 2: Define $W: \Re_{+} \times \bar{\Re}_{+} \rightarrow \bar{\Re}_{+}$by

$$
W(c, m)= \begin{cases}c^{e}+\frac{d}{h}\left((1+m)^{h}-1\right) & \text { if } \quad h \neq 0 \\ c^{e}+d \ln (1+m) & \text { if } \quad h=0\end{cases}
$$

where $e \in(0,1], d \in(0,1)$, and $h<1$. Define $M$ as in Utility Example 1, and define $U$ by (1).

Note that Utility Example 1 would be recovered if $h=1$ were inserted into this functional form. In that case, the discounting function, $d m$, is linear. In this case, $h<1$ and the discounting function, $(d / h)\left((1+m)^{h}-1\right)$ or $d \ln (1+m)$, is concave. Thus greater mean future utility $m$ implies greater impatience. Conveniently, for any value of $h, d$ is the derivative of the discounting function at $m=0$ and the normalization $W(0,0)=0$ is maintained.

The case $h=0$ was introduced by Koopmans, Diamond, and Williamson (1964), and the parametric case $h \in(0,1)$ was introduced in Streufert (1990). Power utility has never been introduced into a stochastic context before, and the case $h<0$ is altogether new.

Proposition 4: $U$ is real-valued and the assumptions of Theorems $B$ and $C$ are met, given Utility Example 2 together with Technology Example 1, 2, or 3.

This proposition is remarkable in that all the tools of dynamic programming are available without any restrictions linking technology parameters to discounting parameters. Power utility is also attractive in the sense that assumptions of increasing impatience are used by Lucas and Stokey 
(1984) and Benhabib, Jafarey, and Nishimura (1988) to guarantee the stability of dynamic general equilibrium.

\section{Nonpositive Objectives}

This section constructs a nonpositive-valued utility function $U$ from a dynamic aggregator $W$ and a stochastic aggregator $M$. Section 4.1 develops assumptions for $W$ and $M$ which are sufficient to imply assumptions U1, U2, and U4-U7 of Section 2's general theory. Sections 4.2 and 4.3 exhibit broad classes of interesting examples which satisfy these assumptions and to which Section 2 may consequently be applied.

\subsection{Sufficient Conditions}

Suppose that a dynamic aggregator $W: \Re_{+} \times \bar{\Re}_{-} \rightarrow \bar{\Re}_{-}$and a stochastic aggregator $M: S \times L\left(S, \bar{\Re}_{-}\right) \rightarrow \bar{\Re}_{-}$satisfy

B1. $W$ is weakly increasing,

B2. $W$ is u.s.c. ,

B3. $(\forall s)\left(\forall u^{\prime} \geq u\right) \quad M_{s}\left(u^{\prime}\right) \geq M_{s}(u)$,

B4. $(\forall s)(\forall y)\left(\forall\right.$ weakly decreasing $\left.\left\langle u^{n}\right\rangle_{n=1}^{\infty} \leq W(\bar{F}(y), 0)\right) \quad \lim _{n \rightarrow \infty} M_{s} u^{n}=M_{s} \lim _{n \rightarrow \infty} u^{n}$, and B5. $(\forall t)\left(\forall u_{t+1} \in L\left(S^{t+1}, \bar{\Re}_{-}\right)\right)$the map ${ }_{1} s_{t} \mapsto M_{s_{t}} u_{t+1}\left({ }_{1} s_{t}, \cdot\right)$ is in $L\left(S^{t}, \bar{\Re}_{-}\right)$.

Note that the inequality restricting $\left\langle u^{n}\right\rangle_{n=1}^{\infty}$ increases the generality of B4 (in fact few of our examples would satisfy B4 if this restriction were removed). Also note that the map in B5 could be suggestedly labeled $u_{t}$.

Define $U: S \times C \rightarrow \bar{\Re}_{-}$by

$$
(\forall s)(\forall c) \quad U_{s}(c)=\lim _{t \rightarrow \infty} W\left(c_{0}, M_{s} W\left(c_{1}, M \cdots W\left(c_{t}, 0\right) \cdots\right)\right) .
$$

The well-definition of $U$ is among the conclusions of the following theorem.

Theorem E: Assume F1 and B1-B5. Then $U$ is well-defined and satisfies U1, U2, and U4-U7. (Proof E.1.)

\subsection{Generalized Isoelastic Preferences}


Utility Example 3: Define $W: \Re_{+} \times \bar{\Re}_{-} \rightarrow \bar{\Re}_{-}$by $W(c, m)=-c^{e}+d m$ where $e<0$ and $d>0$. Define $M$ by

$$
M_{s}(u)=\left\{\begin{array}{lll}
-\left(E_{s}(-u)^{a}\right)^{1 / a} & \text { if } & a \neq 0 \\
-\exp \left(E_{s} \ln (-u)\right) & \text { if } & a=0
\end{array}\right.
$$

where $a \in \Re$. Define $U$ by (4).

The reward function $-c^{e}$ becomes increasingly concave as $e$ becomes more negative (moderate concavity and linearity are accommodated in Utility Example 1). Note that the discount factor $d$ can exceed unity (hence this example subsumes the "upcounted" time-additive deterministic model of Kocherlakota, 1990). When $a \in(1,+\infty), M$ is concave and risk aversion is more than intertemporal inelasticity. When $a \in(-\infty, 1), M$ is convex and risk aversion is less than intertemporal inelasticity (hence $a=0$ results in "logarithmic convexity"). Note that this interpretation of the parameter $a$ reverses that of Utility Examples 1 and 2 because here utility is negative.

Proposition 5: The assumptions of Theorems $A$ and $E$ are met by Utility Example 3 together with Technology Example 1, 2, or 3. (Corollary of Lemma E.3.)

\subsection{Power Discounting}

Utility Example 4: Define $W: \Re_{+} \times \bar{\Re}_{-} \rightarrow \bar{\Re}_{-}$by

$$
W(c, m)= \begin{cases}-c^{e}-\frac{d}{h}\left((1-m)^{h}-1\right) & \text { if } h \neq 0 \\ -c^{e}-d \ln (1-m) & \text { if } h=0\end{cases}
$$

where $e<0, d>0$, and $h \in \Re$. Define $M$ as in Utility Example 3, and define $U$ by (4).

If $h=1$, the discounting function, $d m$, is linear. If $h>1$, the discounting function, $-(d / h)\left((1-m)^{h}-1\right)$, is concave and thus greater future mean utility implies more impatience. Conversely, if $h<1$, the discounting function, $-(d / h)\left((1-m)^{h}-1\right)$ or $-d \ln (1-m)$, is convex and thus greater future mean utility implies less impatience (hence the case $h=0$ results in "logarithmic convexity" and bears an inverted resemblance to the Koopmans-Diamond-Williamson aggregator for nonnegative utility numbers). However, for any value of $h, d$ is the derivative of the discounting function at $m=0$ and the normalization $W(0,0)=0$ is maintained. As in Utility Example 3,d can exceed unity.

This example introduces power discounting to nonpositive preferences (there is no precedent in either a deterministic or stochastic setting). This example could well be used to test the hypothesis that impatience increases with wealth over against the alternative that impatience decreases 
with wealth. The stability of dynamic equilibrium and the long-run distribution of wealth among consumers would hinge critically on the result.

Proposition 6: The assumptions of Theorems $A$ and $E$ are met by Utility Example \& together with Technology Example 1, 2, or 3. (Corollary of Lemma E.3.) 


\section{Appendix A: Lemmas and Proofs for Section 2 (General Theory)}

Lemma A.1: Assume F1 and take any $y$. Then ${ }_{0} \bar{F}(y) \in C$. Further suppose $c$ is feasible from $y$ and $\boldsymbol{x}$ is its associated portfolio process. Then $(\forall t \geq 1) F\left(x_{t-1}\right) \leq \bar{F}^{t}(y)$, and consequently, $(\forall t) c_{t} \leq \bar{F}^{t}(y)$.

Proof: $\bar{F} \in L\left(S \times \Re_{+}, \Re_{+}\right)$because it is upper semi-continuous by $F 1$ and the Maximum Theorem (Berge, 1962, p. 116, Theorem 2). Thus, for any $t$, the composition $\bar{F}^{t}(y)$ belongs to $L\left(S^{t}, \Re_{+}\right)$. Consequently, ${ }_{0} \bar{F}(y) \in \Pi_{t} L\left(S^{t}, \Re_{+}\right)$.

We prove $(\forall t \geq 1) F\left(x_{t-1}\right) \leq \bar{F}^{t}(y)$ by induction. At $t=1$, the definition of $\bar{F}$, the nonnegativity of consumption, and the feasibility imply $F\left(x_{0}\right) \leq \bar{F}\left(\sum_{a} x_{0}^{a}\right) \leq \bar{F}\left(c_{0}+\sum_{a} x_{0}^{a}\right) \leq \bar{F}(y)$. At $t \geq 2$, the definition of $\bar{F}$, the nonnegativity of consumption, feasibility, and the inductive hypothesis imply $F\left(x_{t-1}\right) \leq$ $\bar{F}\left(\sum_{a} x_{t-1}^{a}\right) \leq \bar{F}\left(c_{t-1}+\sum_{a} x_{t-1}^{a}\right) \leq \bar{F}\left(F\left(x_{t-2}\right)\right) \leq \bar{F}\left(\bar{F}^{t-1}(y)\right)=\bar{F}^{t}(y)$.

The inequality $(\forall t) c_{t} \leq \bar{F}^{t}(y)$ follows from the nonnegativity of investment, feasibility, and the previous paragraph: $(\forall t) c_{t} \leq c_{t}+\sum_{a} x_{t}^{a} \leq F\left(x_{t-1}\right) \leq \bar{F}^{t}(y)$.

Lemma A.2: Assume F1, U5, U6, U7, and U8, and suppose that $J^{\circ}$ is admissible. Then the function $J: S \times \Re_{+} \rightarrow T$ defined by

$$
(\forall s, y) J_{s}(y)=\max \left\{W\left(c, M_{s} J^{\circ} \circ F(x)\right) \mid c+\Sigma_{a} x^{a} \leq y\right\}
$$

exists and u.s.c. Furthermore, there exists a measurable function $k: S \times \Re_{+} \rightarrow \Re_{+} \times \Re_{+}^{A}$ such that

$$
(\forall s, y) \quad k_{s}(y) \in \operatorname{argmax}\left\{W\left(c, M_{s} J^{\circ} \circ F(x)\right) \mid c+\Sigma_{a} x^{a} \leq y\right\}
$$

Proof: The feasible set $\left\{(c, x) \mid c+\Sigma_{a} x^{a} \leq y\right\}$ is clearly u.s.c. in $y .{ }^{24}$ The objective function defining $J$ is also u.s.c. because given any $\left(\left(c^{n}, s^{n}, x^{n}\right)\right)_{n=1}^{\infty} \rightarrow\left(c^{0}, s^{0}, x^{0}\right)$ we have

$$
\begin{aligned}
& \varlimsup_{n \rightarrow \infty} W\left(c^{n}, M_{s n} J^{\circ} \circ F\left(x^{n}\right)\right) \\
\leq & W\left(c^{0}, \varlimsup_{n \rightarrow \infty} M_{s, n} J^{\circ} \circ F\left(x^{n}\right)\right) \\
\leq & W\left(c^{0}, M_{s} \circ \varlimsup_{n \rightarrow \infty} J^{\circ} \circ F\left(x^{n}\right)\right) \\
\leq & W\left(c^{0}, M_{s} \circ J^{\circ} \circ F\left(x^{0}\right)\right) .
\end{aligned}
$$

The first inequality follows from U5 and U6; the second from U5 and U8 (note $(\forall n) J^{\circ} \circ F\left(x^{n}\right) \leq J^{\circ} \circ$ $F\left(\vee_{n=1}^{\infty} x^{n}\right) \leq J^{\circ} \circ \bar{F}\left(\sum_{a}\left(\vee_{n=1}^{\infty} x^{n}\right)^{a}\right) \leq J^{+} \circ \bar{F}\left(\sum_{a}\left(\vee_{n=1}^{\infty} x^{n}\right)^{a}\right)$ by the definition of $\bar{F}$ and the admissibility of $J^{\circ}$ ); and the third from U5, U7 and the fact that $J^{\circ} \circ F$ is u.s.c. (by F1 and the admissibility of $J^{\circ}$ ). Hence $J$ exists and is u.s.c. by the Maximum Theorem (Berge, 1963, p. 116, Theorem 2). The existence of a measurable selection $k$ follows immediately by Wagner (1977, p. 880, Theorem 9.1 (ii)).

Lemma A.3: Given F1, U1-U2, and Uł-U8, $\mathrm{J}^{+}$is admissible.

\footnotetext{
24 We employ Berge's (1963, p. 109) concept of upper semi-continuity, which includes compact-valuedness.
} 
Proof: Clearly, $J^{+}$is weakly increasing in $y$ (by U1) and satisfies $J^{-} \leq J^{+} \leq J^{+}$. To show $J^{+}$is u.s.c., take any $\left(\left(s^{n}, y^{n}\right)\right\rangle_{n=1}^{\infty} \rightarrow\left(s^{0}, y^{0}\right)$. Let $\hat{y}>\sup _{n} y^{n}$. For all $t$, we have

$$
\begin{aligned}
& \varlimsup_{n \rightarrow \infty} J_{s^{n}}^{+}\left(y^{n}\right) \\
& =\overline{\lim }_{n \rightarrow \infty} U_{s^{n}}\left(0 \bar{F}\left(y^{n}\right)\right) \\
& \leq \overline{\lim }_{n \rightarrow \infty} U_{s^{n}}\left({ }_{0} \bar{F}_{t-1}\left(y^{n}\right),{ }_{t} \bar{F}(\widehat{y})\right) \\
& =\overline{\lim }_{n \rightarrow \infty} W\left(y^{n}, M_{s} \mathrm{~W}\left(\bar{F}\left(y^{n}\right), M \cdots W\left(\bar{F}^{t-1}\left(y^{n}\right), M U\left({ }_{t} \bar{F}(\widehat{y})\right)\right) \cdots\right)\right) \\
& \leq W\left(\lim _{n \rightarrow \infty} y^{n}, \overline{\lim }_{n \rightarrow \infty} M_{s^{n}} W\left(\bar{F}\left(y^{n}\right), M \cdots W\left(\bar{F}^{t-1}\left(y^{n}\right), M U\left({ }_{t} \bar{F}(\widehat{y})\right)\right) \cdots\right)\right) \\
& =W\left(y^{0}, \overline{\lim }_{n \rightarrow \infty} M_{s^{n}} W\left(\bar{F}\left(y^{n}\right), M \cdots W\left(\bar{F}^{t-1}\left(y^{n}\right), M U\left({ }_{t} \bar{F}(\hat{y})\right)\right) \cdots\right)\right) \\
& \leq W\left(y^{0}, M_{s} 0 \varlimsup_{n \rightarrow \infty} W\left(\bar{F}\left(y^{n}\right), M \cdots W\left(\bar{F}^{t-1}\left(y^{n}\right), M U\left({ }_{t} \bar{F}(\hat{y})\right)\right) \cdots\right)\right) \\
& \leq W\left(y^{0}, M_{\mathrm{s}} \mathrm{o} W\left(\overline{\lim }_{n \rightarrow \infty} \bar{F}\left(y^{n}\right), \overline{\lim }_{n \rightarrow \infty} M \cdots W\left(\bar{F}^{t-1}\left(y^{n}\right), M U\left({ }_{t} \bar{F}(\widehat{y})\right)\right) \cdots\right)\right) \\
& \leq W\left(y^{0}, M_{s} \circ W\left(\bar{F}\left(y^{0}\right), \overline{\lim }_{n \rightarrow \infty} M \cdots W\left(\bar{F}^{t-1}\left(y^{n}\right), M U\left({ }_{t} \bar{F}(\widehat{y})\right)\right) \cdots\right)\right) \\
& \leq W\left(y^{0}, M_{s} \circ W\left(\bar{F}\left(y^{0}\right), M \overline{\lim }_{n \rightarrow \infty} \cdots W\left(\bar{F}^{t-1}\left(y^{n}\right), M U\left({ }_{t} \bar{F}(\hat{y})\right)\right) \cdots\right)\right) \\
& \text {... } \\
& \leq W\left(y^{0}, M_{s} \circ W\left(\bar{F}\left(y^{0}\right), M \cdots W\left(\bar{F}^{t-1}\left(y^{0}\right), M U\left({ }_{t} \bar{F}(\widehat{y})\right)\right) \cdots\right)\right) \\
& =U_{s} 0\left({ }_{0} \bar{F}_{t-1}\left(y^{0}\right),{ }_{t} \bar{F}(\hat{y})\right) \text {. }
\end{aligned}
$$

The first line follows from the definition of $\mathrm{J}^{+}$; the next from U1; and the third from U4. In the second group, the first follows from U5 and U6; the second from the definition of $y^{n}$; and the third from U5 and U8 (at each $n$, the utility argument of $M$ is bounded by $J^{+} \circ \bar{F}(\widehat{y})$ ). In the third group, the first follows from $\mathrm{U} 5, \mathrm{U} 6, \mathrm{U} 7$, and the increase of $W$ in its first argument (by $\mathrm{U} 1$ and $\mathrm{U} 4$ ); the second from $\mathrm{U} 5, \mathrm{U} 7$, and the u.s.c. of $\bar{F}$; and the third from U5, U7, and U8 (at each $n$ and each $s_{1}$, the argument of $M_{s_{1}}$ is bounded by $\left.J^{+} \circ \bar{F} \circ \bar{F}_{s_{1}}(\widehat{y})\right)$. By a similar group of inequalities at time periods $2,3, \ldots, t-1$, we arrive at the final inequality. The final equality holds by $\mathrm{U} 4$. Since this mammoth inequality holds for all $t$, it must be that

$$
\begin{aligned}
& \varlimsup_{\lim _{n \rightarrow \infty} J_{s^{n}}^{+}\left(y^{n}\right)} \\
\leq & \lim _{t \rightarrow \infty} U_{s^{0}}\left({ }_{0} \bar{F}_{t-1}\left(y^{0}\right), t \bar{F}(\widehat{y})\right) \\
= & U_{s} 0\left(0 \bar{F}\left(y^{0}\right)\right) \\
= & J_{s^{0}}^{+}\left(y^{0}\right) .
\end{aligned}
$$

The first equality holds by the upper convergence (U2) of $U$ at $s=s^{0}$ and $y=\hat{y}$, and the second by the definition of $\mathrm{J}^{+}$.

Lemma A.4: Assume U1, U4, U5, U6, and U8. Then $\mathrm{J}^{-}$is admissible. 
Proof: Clearly $J^{-}$is weakly increasing in $y$ and satisfies $J^{-} \leq J^{-} \leq J^{+}$. $J^{-}$is u.s.c. because if $\left\langle\left(s^{n}, y^{n}\right)\right\rangle_{n=1}^{\infty} \rightarrow\left(s^{0}, y^{0}\right)$, then $\overline{\lim }_{n \rightarrow \infty} J_{s^{n}}^{-}\left(y^{n}\right)=\overline{\lim }_{n \rightarrow \infty} U_{s^{n}}(0)=\overline{\lim }_{n \rightarrow \infty} W\left(0, M_{s^{n}} U\left({ }_{1} 0\right)\right) \leq$ $W\left(0, \overline{\lim }_{n \rightarrow \infty} M_{s} \mathrm{n}\left({ }_{1} 0\right)\right) \leq W\left(0, M_{s} \mathrm{o} \overline{\lim }_{n \rightarrow \infty} U\left({ }_{1} 0\right)\right)=W\left(0, M_{s} \mathrm{o} U\left({ }_{1} 0\right)\right)=U_{s^{0}}(0)=J_{s^{0}}^{-}\left(y^{0}\right)$. These steps follow from the definition of $\mathrm{J}^{-}$; U4; U5 and U6; U5 and U8 (note $U\left({ }_{1} 0\right) \leq J^{+} \circ \bar{F}(0)$ ); the constancy of $U\left({ }_{1} 0\right)$ with respect to $n ; U 4$; and the definition of $J^{-}$.

Lemma A.5: Assume F1, U1-U2, and U4-U8. Then $B$ is well-defined.

Proof: Lemmas A.2, A.3, and A.4 assure the existence of $\max \left\{W\left(c, M_{s} J^{+} \circ F(x)\right) \mid c+\Sigma_{a} x^{a} \leq y\right\}$ and $\max \left\{W\left(c, M_{s} J^{-} \circ F(x)\right) \mid c+\Sigma_{a} x^{a} \leq y\right\}$. Now take any admissible $J^{\circ}$ and define $J: S \times \Re_{+} \rightarrow T$ by

$$
(\forall s, y) J_{s}(y)=\max \left\{W\left(c, M_{s} J^{\circ} \circ F(x)\right) \mid c+\Sigma_{a} x^{a} \leq y\right\}
$$

$J$ exists and is u.s.c. by Lemma A.2. $J$ is weakly increasing in $y$ by expansion of the feasible set $\{(c, x) \mid c+$ $\left.\Sigma_{a} x^{a} \leq y\right\}$ with $y$. Finally, we note

$$
\begin{aligned}
(\forall s, y) & J_{s}^{-}(y) \\
= & U_{s}(0) \\
= & W\left(0, M_{s} U\left({ }_{1} 0\right)\right) \\
\leq & \max \left\{W\left(c, M_{s} U\left({ }_{1} 0\right)\right) \mid c+\Sigma_{a} x^{a} \leq y\right\} \\
= & \max \left\{W\left(c, M_{s} J^{-} \circ F(x)\right) \mid c+\Sigma_{a} x^{a} \leq y\right\} \\
\leq & J_{s}(y) \\
\leq & \max \left\{W\left(c, M_{s} J^{+} \circ F(x)\right) \mid c+\Sigma_{a} x^{a} \leq y\right\} \\
= & \max \left\{W\left(c, M_{s} U\left({ }_{0} \bar{F}(F(x))\right)\right) \mid c+\Sigma_{a} x^{a} \leq y\right\} \\
\leq & W\left(y, M_{s} U\left({ }_{0} \bar{F}(\bar{F}(y))\right)\right) \\
= & U_{s}\left({ }_{0} \bar{F}(y)\right) \\
= & J_{s}^{+}(y) .
\end{aligned}
$$

The second and third inequalities hold by $\mathrm{U} 5, \mathrm{U} 7$, and $J^{-} \leq J^{\circ} \leq J^{+}$(by admissibility). Thus $J=B J^{\circ}$ is admissible.

Proof A.6 (for Theorem A): Since $B J^{+} \leq J^{+}\left(\right.$Lemma A.5), U5 and U7 imply $(\forall n) B^{n}\left(B J^{+}\right) \leq$ $B^{n}\left(J^{+}\right)$and hence $(\forall n) B^{n+1} J^{+} \leq B^{n} J^{+}$. Therefore $\lim _{n \rightarrow \infty} B^{n} J^{+}$exists and is u.s.c. because it is the limit of a weakly decreasing sequence of u.s.c. functions (Lemma A.5). Moreover, $\lim _{n \rightarrow \infty} B^{n} J^{+}$is weakly increasing in $y$ and satisfies $J^{-} \leq \lim _{n \rightarrow \infty} B^{n} J^{+} \leq J^{+}$because each $B^{n} J^{+}$is weakly increasing in $y$ and satisfies $J^{-} \leq B^{n} J^{+} \leq J^{+}$(Lemma A.5). Thus $\lim _{n \rightarrow \infty} B^{n} J^{+}$is admissible. For notational ease, define $J^{\infty}=\lim _{n \rightarrow \infty} B^{n} J^{+}$.

This paragraph shows that $J^{\infty}=B J^{\infty}$ by deriving (a) $J^{\infty} \geq B J^{\infty}$ and (b) $J^{\infty} \leq B J^{\infty}$. (a) If 
$J^{\infty} \geq B J^{\infty}$ were false, there would be $(s, y)$ such that $J_{s}^{\infty}(y)<B J_{s}^{\infty}(y)$. Thus by the definition of $J^{\infty}$ (applied twice), there would be an $n \geq 1$ such that $B^{n} J^{+}{ }_{s}(y)<B J^{\infty}(y) \leq B \circ B^{n} J^{+}{ }_{s}(y)=B^{n+1} J^{+}{ }_{s}(y)$. This would contradict the weak decrease of $\left\langle B^{n} J^{+}\right\rangle_{n=1}^{\infty}$. (b) Take any $(s, y)$. By the existence of $B$ (Lemma A.5), there is a sequence $\left(\left(c^{n}, x^{n}\right)\right)_{n=0}^{\infty}$ such that

$$
(\forall n \geq 0)\left(c^{n}, x^{n}\right) \in \operatorname{argmax}\left\{W\left(c, M_{s} B^{n} J^{+} \circ F(x)\right) \mid c+\Sigma_{a} x^{a} \leq y\right\} .
$$

Since $\left\{(c, x) \mid c+\Sigma_{a} x^{a} \leq y\right\}$ is compact, there exists a subsequence $\left\langle\left(c^{\eta(m)}, x^{\eta(m)}\right)\right\rangle_{m=1}^{\infty}$ which converges to some $(\widehat{c}, \widehat{x}) \in\left\{(c, x) \mid c+\Sigma_{a} x^{a} \leq y\right\}$. We claim

$$
\begin{aligned}
B J_{s}^{\infty}(y) & =\max \left\{W\left(c, M_{s} J^{\infty} \circ F(x)\right) \mid c+\Sigma_{a} x^{a} \leq y\right\} \\
& \geq W\left(\widehat{c}, M_{s} J^{\infty} \circ F(\widehat{x})\right) \\
& =W\left(\widehat{c}, M_{s} \lim _{\ell \rightarrow \infty} B^{\eta(\ell)} J^{+} \circ F(\widehat{x})\right) \\
& \geq W\left(\widehat{c}, M_{s} \lim _{\ell \rightarrow \infty} \varlimsup_{m \rightarrow \infty} B^{\eta(\ell)} J^{+} \circ F\left(x^{\eta(m)}\right)\right) \\
& \geq W\left(\widehat{c}, M_{s} \lim _{\ell \rightarrow \infty} \varlimsup_{m \rightarrow \infty} B^{\eta(m)} J^{+} \circ F\left(x^{\eta(m)}\right)\right) \\
& =W\left(\widehat{c}, M_{s} \overline{\lim }_{m \rightarrow \infty} B^{\eta(m)} J^{+} \circ F\left(x^{\eta(m)}\right)\right) \\
& \geq W\left(\widehat{c}, \varlimsup_{m \rightarrow \infty} M_{s} B^{\eta(m)} J^{+} \circ F\left(x^{\eta(m)}\right)\right) \\
& \geq \overline{\lim }_{m \rightarrow \infty} W\left(c^{\eta(m)}, M_{s} B^{\eta(m)} J^{+} \circ F\left(x^{\eta(m)}\right)\right) \\
& =\overline{\lim }_{m \rightarrow \infty} B^{\eta(m)+1} J_{s}^{+}(y) \\
& =\lim _{m \rightarrow \infty} B^{\eta(m)+1} J_{s}^{+}(y) \\
& =J_{s}^{\infty}(y) .
\end{aligned}
$$

These steps follows from the definition of $B ;(\widehat{c}, \widehat{x}) \in\left\{(c, x) \mid c+\Sigma_{a} x^{a} \leq y\right\}$; the definition of $J^{\infty}$; the u.s.c. of each $B^{\eta(\ell)} J^{+} \circ F ;(\forall m \geq \ell) B^{\eta(m)} J^{+} \leq B^{\eta(\ell)} J^{+}$; the expression is constant with respect to $\ell ; \mathrm{U} 8$ and the fact that $\left\langle B^{\eta(m)} J^{+} \circ F\left(x^{\eta(m)}\right)\right\rangle_{m=1}^{\infty}$ is bounded from above by $\left\langle J^{+} \circ F\left(x^{\eta(m)}\right)\right\rangle_{m=1}^{\infty} \leq J^{+} \circ F\left(\sum_{a}\left(V_{m=1}^{\infty} x^{\eta(m)}\right)^{a}\right)$; U5 and U6; the definition of $\left\langle\left(c^{n}, x^{n}\right)\right\rangle_{n=1}^{\infty}$; the weak decrease of $\left\langle B^{n} J^{+}\right\rangle_{n=1}^{\infty}$; and the definition of $J^{\infty}$.

Let $J^{\prime}$ be any admissible solution to Bellman's equation. Note that $(\forall n) J^{\prime} \leq B^{n} J^{+}$because $(\forall n) B^{n} J^{\prime} \leq B^{n} J^{+}$(since $J^{\prime} \leq J^{+}$by admissibility) and because $(\forall n) J^{\prime}=B^{n} J^{\prime}$ (since $J^{\prime}$ solves Bellman's equation). Thus $J^{\prime} \leq \lim _{n \rightarrow \infty} B^{n} J^{+} \equiv J^{\infty}$. Thus far we have shown that $J^{\infty}$ exists and that it is the greatest admissible solution to Bellman's equation.

Take any $(s, y)$ and any $(c, x)$ which is feasible from $(s, y)$. Note that

$$
\begin{aligned}
(\forall t \geq 1) & U_{s}(c) \\
\leq & U_{s}\left({ }_{0} c_{t},{ }_{0} \bar{F}\left(F\left(x_{t}\right)\right)\right) \\
= & W\left(c_{0}, M_{s} W\left(c_{1}, M \cdots W\left(c_{t}, M U\left({ }_{0} \bar{F}\left(F\left(x_{t}\right)\right)\right)\right) \cdots\right)\right) \\
= & W\left(c_{0}, M_{s} W\left(c_{1}, M \cdots W\left(c_{t}, M J^{+} \circ F\left(x_{t}\right)\right) \cdots\right)\right) \\
\leq & W\left(c_{0}, M_{s} W\left(c_{1}, M \cdots B J^{+} \circ F\left(x_{t-1}\right) \cdots\right)\right)
\end{aligned}
$$




$$
\begin{aligned}
& \leq W\left(c_{0}, M_{s} B^{t} J^{+} \circ F\left(x_{0}\right)\right) \\
& \leq B^{t+1} J_{s}^{+}(y)
\end{aligned}
$$

by $\mathrm{U} 1$ and the feasibility of $(c, x)$; U4; the definition of $J^{+}$; and $t+1$ applications of the definition of $B$. Thus $U_{s}(c) \leq J_{s}^{\infty}(y) \equiv \lim _{n \rightarrow \infty} B^{n} J_{s}^{+}(y)$. We conclude that, for any $(s, y)$, no feasible consumption plan attains a utility higher than $J_{s}^{\infty}(y)$.

By Lemma A.2 and the admissibility of $J^{\infty}$, there exists a measurable function $k^{\infty}: S \times \Re_{+} \rightarrow$ $\Re_{+} \times \Re_{+}^{A}$ such that

$$
(\forall s, y) k_{s}^{\infty}(y) \in \operatorname{argmax}\left\{W\left(c, M_{s} J^{\infty} \circ F(x)\right) \mid c+\Sigma_{a} x^{a} \leq y\right\}
$$

Now take any $(s, y)$, let $\left(c_{0}^{\infty}, x_{0}^{\infty}\right)=k_{s}^{\infty}(y)$, and let $(\forall t \geq 1)\left(c_{t}^{\infty}, x_{t}^{\infty}\right)=k^{\infty} \circ F\left(x_{t-1}^{\infty}\right)$ (the measurability of $k^{\infty}$ implies $\left.c^{\infty} \in \Pi_{t=0}^{\infty} L_{+}\left(S^{t}, \Re_{+}\right)\right)$. By (5) and $J^{\infty}=B J^{\infty}$, we have

$$
J_{s}^{\infty}(y)=W\left(c_{0}^{\infty}, M_{s} J^{\infty} \circ F\left(x_{0}^{\infty}\right)\right) \text { and }
$$

$$
(\forall t \geq 1) J^{\infty} \circ F\left(x_{t-1}^{\infty}\right)=W\left(c_{t}^{\infty}, M J^{\infty} \circ F\left(x_{t}^{\infty}\right)\right) \text {. }
$$

Note that

$$
\begin{aligned}
(\forall t \geq 1) J_{s}^{\infty}(y) & =W\left(c_{0}^{\infty}, M_{s} W\left(c_{1}^{\infty}, M \cdots W\left(c_{t}^{\infty}, M J^{\infty} \circ F\left(x_{t}^{\infty}\right)\right) \cdots\right)\right) \\
& \leq W\left(c_{0}^{\infty}, M_{s} W\left(c_{1}^{\infty}, M \cdots W\left(c_{t}^{\infty}, M J^{+} \circ F\left(x_{t}^{\infty}\right)\right) \cdots\right)\right) \\
& =W\left(c_{0}^{\infty}, M_{s} W\left(c_{1}^{\infty}, M \cdots W\left(c_{t}^{\infty}, M U\left({ }_{0} \bar{F}\left(F\left(x_{t}^{\infty}\right)\right)\right)\right) \cdots\right)\right) \\
& \leq W\left(c_{0}^{\infty}, M_{s} W\left(c_{1}^{\infty}, M \cdots W\left(c_{t}^{\infty}, M U\left({ }_{t+1} \bar{F}(y)\right)\right) \cdots\right)\right) \\
& =U_{s}\left({ }_{0} c_{t}^{\infty},{ }_{t+1} \bar{F}(y)\right)
\end{aligned}
$$

by (6); the admissibility of $J^{\infty}$; the definition of $J^{+} ; \mathrm{U} 1$; and U4. Therefore upper convergence at $y$ implies

$$
\begin{aligned}
J_{s}^{\infty}(y) & \leq \lim _{t \rightarrow \infty} U_{s}\left(0 c_{t}^{\infty}, t_{t+1} \bar{F}(y)\right) \\
& =U_{s}\left(c^{\infty}\right) .
\end{aligned}
$$

Thus we conclude that, for any $(s, y)$, there exists a feasible stream which attains a utility of at least $J_{s}^{\infty}(y)$. This and the conclusion of the preceding paragraph demonstrate that $J^{\infty}=J^{*}$.

Finally, take any $(s, y)$. Any recursively optimal $(c, x)$ must satisfy $(6)$ (recall $J^{\infty}=J^{*}$ ) and thus the remainder of the preceding paragraph demonstrates that $J_{s}^{*}(y) \leq U_{s}(c)$. Hence $(c, x)$ is optimal. We conclude that recursive optimality implies optimality.

Proof A.7 (for Theorem B): We know $\lim _{n \rightarrow \infty} B^{n} J^{-} \leq J^{*}$ since $\lim _{n \rightarrow \infty} B^{n} J^{-} \leq \lim _{n \rightarrow \infty} B^{n} J^{+}$ by $J^{-} \leq J^{+}$, and since $\lim _{n \rightarrow \infty} B^{n} J^{+}=J^{*}$ by Theorem A. Suppose $\lim _{n \rightarrow \infty} B^{n} J^{-} \geq J^{*}$ was false. Then 
there would be a $(s, y)$ and a feasible $\left(c^{*}, x^{*}\right)$ such that $\lim _{n \rightarrow \infty} B^{n} J^{-}(y)<U_{s}\left(c^{*}\right)$. Lower convergence at $y$ would then imply the existence of a time period $t$ such that

$$
\lim _{n \rightarrow \infty} B^{n} J^{-}(y)<U_{s}\left({ }_{0} c_{t-1}^{*}, t 0\right)
$$

This leads to a contradiction:

$$
\begin{aligned}
& U_{s}\left({ }_{0} c_{t-1}^{*},{ }_{t} 0\right) \\
> & B^{t} J_{s}^{-}(y) \\
= & \max \left\{W\left(c_{0}, M_{s} B^{t-1} J^{-} \circ F\left(x_{0}\right)\right) \mid c_{0}+\Sigma_{a} x_{0}^{a} \leq y\right\} \\
= & \max \left\{W\left(c_{0}, M_{s} \max \left\{W\left(c_{1}, M B^{t-2} J^{-} \circ F\left(x_{1}\right)\right) \mid c_{1}+\Sigma_{a} x_{1}^{a} \leq F\left(x_{0}\right)\right\} \mid c_{0}+\Sigma_{a} x_{0}^{a} \leq y\right\}\right. \\
= & \max \left\{W\left(c_{0}, M_{s} W\left(c_{1}, M B^{t-2} J^{-} \circ F\left(x_{1}\right)\right) \mid(c, x) \text { feasible }\right\}\right. \\
& \cdots \\
= & \max \left\{W\left(c_{0}, M_{s} W\left(c_{1}, M \cdots W\left(c_{t-1}, M J^{-} \circ F\left(x_{t-1}\right)\right) \cdots\right)\right) \mid(c, x) \text { feasible }\right\} \\
\geq & W\left(c_{0}^{*}, M_{s} W\left(c_{1}^{*}, M \cdots W\left(c_{t-1}^{*}, M J^{-} \circ F\left(x_{t-1}^{*}\right)\right) \cdots\right)\right) \\
= & U_{s}\left(\circ c_{t-1}^{*},{ }_{t} 0\right)
\end{aligned}
$$

The strict inequality follows from (8) and the weak increase of $\left(B^{t} J^{-}\right\rangle_{t=1}^{\infty}$, and the first equality from the definition of $B$. The next two steps follow from the definition of $B$, and from $\mathrm{U} 5$ and $\mathrm{U} 7$; and the succeeding equality is obtained by repeating these two steps in the $t-2$ time periods $\{2,3, \ldots, t-1\}$. The last inequality holds by the feasibility of $\left(c^{*}, x^{*}\right)$, and the last equality by $U 4$ and the definition of $J^{-}$. Thus we conclude that $\lim _{n \rightarrow \infty} B^{n} J^{-}=J^{*}$.

Let $J^{\prime}$ be any admissible solution to Bellman's equation. Note that $(\forall n) J^{\prime} \geq B^{n} J^{-}$because $(\forall n) B^{n} J^{\prime} \geq B^{n} J^{-}$(since $J^{\prime} \geq J^{-}$by admissibility) and because $(\forall n) J^{\prime}=B^{n} J^{\prime}$ (since $J^{\prime}$ solves Bellman's equation). Thus $J^{\prime} \geq \lim _{n \rightarrow \infty} B^{n} J^{-}$. Since $\lim _{n \rightarrow \infty} B^{n} J^{-}=J^{*}$ by the previous paragraph, $J^{*}$ must therefore be the least admissible solution to Bellman's equation. Since $J^{*}$ is also the greatest solution by Theorem A, it must be the unique solution.

The remaining conclusions follow from Theorem A.

Proof A.8 (for Theorem C): Notice that $\left\{(c, x) \mid c+\Sigma_{a} x^{a} \leq y\right\}$ is continuous in $y$. Also notice that the function $(c, s, x) \mapsto W\left(c, M_{s} J^{\circ} \circ F(x)\right)$ is continuous whenever $J^{\circ}$ is continuous and admissible: it is u.s.c. by the argument given in the proof of Lemma A.2; and it is l.s.c. by a symmetric argument using U9, U10, the 1.s.c. of $J^{\circ}$, and F2.

$J^{-}$is continuous: it is u.s.c. by the argument given in the proof of Lemma A.4; it is l.s.c. by a symmetric argument using $\mathrm{U} 9$ and $\mathrm{U} 10$. If $B^{n} J^{-}$is continuous, then $B^{n+1} J^{-}$is continuous: this follows from the first paragraph and the Maximum Theorem (Berge, 1963, p.116). Thus every $B^{n} J^{-}$is continuous.

$J^{*}$ is continuous: it is u.s.c. because it is admissible (Theorem A); it is l.s.c. since it is the limit of a 
weakly increasing sequence of l.s.c. functions (previous paragraph). Finally, $K^{*}$ is u.s.c. ${ }^{25}$ : this follows from the first paragraph, the continuity of $J^{*}$, and the Maximum Theorem.

\section{Appendix B: Continuous Monotonic Transformations}

Section 2 assumes $T \subset \bar{\Re}, U: S \times C \rightarrow T, W: \Re_{+} \times T \rightarrow T$, and $M: S \times L(S, T) \rightarrow T$. Section 3 sets $T=\bar{\Re}_{+}$, assumes $W$ and $M$, and derives $U$. Similarly, Section 4 sets $T=\bar{\Re}_{-}$, assumes $W$ and $M$, and derives $U$. This section considers transformations of the utility set $T$ by means of a continuous, increasing, and invertible function $\phi: T \rightarrow \widehat{T}$. The transformed utility function and aggregators are

$$
\begin{array}{lll}
\widehat{U}: S \times C \rightarrow \widehat{T} & \text { defined by } & (\forall s)(\forall c) \widehat{U}_{s}(c)=\phi U_{s}(c), \\
\widehat{W}: \Re_{+} \times \widehat{T} \rightarrow \widehat{T} & \text { defined by } & (\forall c)(\forall \hat{m}) \widehat{W}(c, \hat{m})=\phi W\left(c, \phi^{-1} \hat{m}\right), \text { and } \\
\widehat{M}: S \times L(S, \widehat{T}) \rightarrow \widehat{T} & \text { defined by } & (\forall s)(\forall \hat{u}) \widehat{M}_{s} \hat{u}=\phi M_{s} \phi^{-1} \hat{u} .
\end{array}
$$

Part (a) of the following lemma shows that the assumptions of Theorems A, B, and C in Section 2 are invariant to continuous monotonic transformation (the conclusions also happen to be invariant as well). Part (b) shows that many of the assumptions and all of the conclusions of Theorem D in Section 3 are also invariant (conditions N1-N12 are not invariant). Part (c) shows that all of the assumptions and conclusions of Theorem $\mathrm{E}$ are invariant.

Lemma B.1: (a) Each of the Properties U1-U10 is satisfied by $[\widehat{U}, \widehat{W}, \widehat{M}]$ iff it is satisfied by $[U, W, M]$.

(b) Suppose $T=\widehat{T}=\bar{\Re}_{+}$and $U$ is defined by (1). Then we have that each of the properties A1-A6 is satisfied by $[\widehat{W}, \widehat{M}]$ iff it is satisfied by $[W, M]$, that

$$
(\forall s)(\forall c) \quad \widehat{U}_{s}(c)=\lim _{t \rightarrow \infty} \widehat{W}\left(c_{0}, \widehat{M}, \widehat{W}\left(c_{1}, \widehat{M} \ldots \widehat{W}\left(c_{t}, 0\right) \cdots\right)\right)
$$

and that $\widehat{U}$ is real-valued iff $U$ is real-valued.

(c) Suppose $T=\widehat{T}=\bar{\Re}_{-}$and $U$ is defined by (4). Then we have that each of the properties B1-B5 is satisfied by $[\widehat{W}, \widehat{M}]$ iff it is satisfied by $[W, M]$, that

$$
(\forall s)(\forall c) \quad \widehat{U}_{s}(c)=\lim _{t \rightarrow \infty} \widehat{W}\left(c_{0}, \widehat{M}, \widehat{W}\left(c_{1}, \widehat{M} \ldots \widehat{W}\left(c_{t}, 0\right) \cdots\right)\right),
$$

and that $\hat{U}$ is real-valued iff $U$ is real-valued.

Proof: The order properties U1, U5, U7, A1, A4, B1, and B3 are obvious from the increase of $\phi$. The continuity and adaptedness properties U2, U3, A3, A6, and B5 are obvious from the continuity of $\phi$. The semi-continuity and monotone convergence properties $\mathrm{U} 6, \mathrm{U} 9, \mathrm{~A} 2, \mathrm{~A} 5, \mathrm{~B} 2$, and $\mathrm{B} 4$ are obvious from the increase and continuity of $\phi$.

\footnotetext{
${ }^{25}$ We employ Berge's (1963, p. 109) concept of upper semi-continuity, which includes compact-valuedness.
} 
U4 is satisfied by $[\widehat{U}, \widehat{W}, \widehat{M}]$ iff it is satisfied by $[U, W, M]$ because

$$
(\forall s)(\forall c) \quad \widehat{W}\left(c_{0}, \widehat{M}_{s} \widehat{U}\left({ }_{1} c\right)\right)=\phi W\left(c_{0}, \phi^{-1} \phi M_{s} \phi^{-1} \phi U\left({ }_{1} c\right)\right)=\phi W\left(c_{0}, M_{s} U\left({ }_{1} c\right)\right) .
$$

U8. We first show that, for any $\bar{u}, \widehat{M}$ is u.q.c. beneath $\phi \bar{u}$ iff $M$ is u.q.c. beneath $\bar{u}$. Assume that $M$ is u.q.c. beneath $\bar{u}$, and take any $\left\langle s^{n}\right\rangle_{n} \rightarrow s^{0}$ and any $\left\langle\hat{u}^{n}\right\rangle_{n} \leq \phi \bar{u}$. Then

$$
\begin{aligned}
& \varlimsup_{\lim _{n \rightarrow \infty}} \widehat{M}_{s^{n}} \hat{u}^{n} \\
= & \varlimsup_{n \rightarrow \infty} \phi M_{s^{n}} \phi^{-1} \hat{u}^{n} \\
= & \phi \varlimsup_{n \rightarrow \infty} M_{s^{n}} \phi^{-1} \hat{u}^{n} \\
\leq & \phi M_{s^{\circ}} \varlimsup_{\lim _{n \rightarrow \infty} \phi^{-1}} \hat{u}^{n} \\
= & \phi M_{s^{\circ} \circ \phi^{-1}} \overline{\lim }_{n \rightarrow \infty} \hat{u}^{n} \\
= & \widehat{M}_{s} \circ \overline{l i m}_{n \rightarrow \infty} \hat{u}^{n} .
\end{aligned}
$$

The second and third equalities hold by the continuity and increase of $\phi$. The inequality holds because $(\forall n) \phi^{-1} \hat{u}^{n}=\phi^{-1} \phi \bar{u} \leq \bar{u}$ and $M$ is u.q.c. beneath $\bar{u}$. The converse holds by a symmetric argument. This equivalence implies that $\mathrm{U} 8$ for $\widehat{M}$ is equivalent to U8 for $M$ : for every $y$, set $\bar{u}=J^{+} \circ \bar{F}(y)$.

U10. In a symmetric manner to the preceding paragraph, one can show that for any $\underline{u}, \widehat{M}$ is l.q.c. above $\phi \underline{u}$ iff $M$ is l.q.c. above $\underline{u}$. This equivalence implies that U10 for $\widehat{M}$ is equivalent to U10 for $M$ : set $\underline{u}=J^{-}(0)$.

The Equality for $\widehat{U}$. Suppose $T=\widehat{T}=\bar{\Re}_{+}$and $U$ is defined by (??). Then

$$
\begin{aligned}
(\forall s)(\forall c) & \widehat{U}_{s}(c) \\
= & \phi U_{s}(c) \\
= & \phi \lim _{t \rightarrow \infty} W\left(c_{0}, M_{s} W\left(c_{1}, M \ldots W\left(c_{t}, 0\right) \cdots\right)\right) \\
= & \lim _{t \rightarrow \infty} \phi W\left(c_{0}, M_{s} W\left(c_{1}, M \ldots W\left(c_{t}, 0\right) \cdots\right)\right) \\
= & \lim _{t \rightarrow \infty} \phi W\left(c_{0}, \phi^{-1} \phi M_{s} \phi^{-1} \phi W\left(c_{1}, \phi^{-1} \phi M \phi^{-1} \ldots \phi W\left(c_{t}, \phi^{-1} 0\right) \cdots\right)\right) \\
= & \lim _{t \rightarrow \infty} \widehat{W}\left(c_{0}, \widehat{M}_{s} \widehat{W}\left(c_{1}, \widehat{M} \ldots \widehat{W}\left(c_{t}, 0\right) \ldots\right)\right) .
\end{aligned}
$$

The third equality holds by the continuity of $\phi$, and the fourth holds because $\phi^{-1}(0)=0$ since $\phi$ is an increasing and invertible function from $\bar{\Re}_{+}$to itself. An identical argument holds when $T=\widehat{T}=\bar{\Re}_{-}$.

Real-valuedness. Suppose $T=\widehat{T}=\bar{\Re}_{+}$. Since $\phi$ is an increasing and invertible function from $\bar{\Re}_{+}$ to itself, both the restriction of $\phi$ to $\Re_{+}$and the restriction of $\phi^{-1}$ to $\Re_{+}$are real-valued. This implies that $\widehat{U}=\phi U$ is real-valued iff $U$ is real-valued. An identical argument holds when $T=\widehat{T}=\bar{\Re}_{-}$.

\section{Appendix C: Quasi-Continuity}

This section considers stochastic aggregators $M: S \times L(S, T) \rightarrow T$ of the form $\int \cdot d Q_{s}$, where $T$ is a closed subset of $\bar{\Re}$ and $Q: S \times B_{S} \rightarrow[0,1]$ is a stochastic kernel, that is, $(\forall s) Q_{s}(\cdot)$ is a probability 
measure and $(\forall E)$ Q. $(E)$ is measurable. Conditions M1 and M2 of Lemma C.1 are sufficient for upper quasi-continuity in this special case. Note that if $Q$ is constant with respect to $s$, then $\mathrm{M} 1$ is satisfied and M2 is equivalent to the integrability of $\bar{u} \vee 0$. Also note that M2 is satisfied whenever $\bar{u}$ is bounded from above by a (finite) constant.

Lemma C.1 (U.Q.C.): $\int \cdot d Q$, is u.q.c. beneath $\bar{u} \in L(S, T)$ if $(Q, \bar{u})$ satisfies:

$$
\begin{aligned}
& \text { M1. }\left(\forall\left\langle s^{n}\right\rangle_{n=1}^{\infty} \rightarrow s^{0}\right) \quad \sup _{E \in \mathcal{B}_{s}}\left|Q_{s^{n}}(E)-Q_{s^{0}}(E)\right| \rightarrow 0 \text { and } \\
& \text { M2. } \quad\left(\forall\left\langle s^{n}\right\rangle_{n=1}^{\infty} \rightarrow s^{0}\right) \quad \lim _{b \rightarrow \infty} \overline{\lim }_{n \rightarrow \infty} \int_{\{\bar{u} \geq b\}} \bar{u}\left(s^{\prime}\right) Q_{s^{n}}\left(d s^{\prime}\right)=0 .
\end{aligned}
$$

Proof: This paragraph proves the claim when $\left\langle u^{n}\right\rangle_{n=1}^{\infty}$ is uniformly bounded both from above and from below. Let $s^{n} \rightarrow s^{0}$. Then

$$
\begin{aligned}
& \varlimsup_{n \rightarrow \infty} \int_{S} u^{n} d Q_{s^{n}} \\
\leq & \varlimsup_{n \rightarrow \infty}\left[\int_{S} u^{n} d Q_{s^{n}}-\int_{S} u^{n} d Q_{s^{0}}\right]+\varlimsup_{n \rightarrow \infty} \int_{S} u^{n} d Q_{s^{\circ}} \\
\leq & \varlimsup_{n \rightarrow \infty}\left[\int_{S} u^{n} d Q_{s^{n}}-\int_{S} u^{n} d Q_{s^{0}}\right]+\int_{S} \varlimsup_{n \rightarrow \infty} u^{n} d Q_{s^{\circ}} \\
= & 0+\int_{S} \varlimsup_{n \rightarrow \infty} u^{n} d Q_{s^{\circ}}
\end{aligned}
$$

The second inequality follows by Fatou's lemma. The equality follows by M1 and the assumption that $\left\langle u^{n}\right\rangle$ is uniformly bounded (for example, see Stokey and Lucas, 1989, p. 341, Theorem 11.7).

This paragraph proves the claim when $\left\langle u^{n}\right\rangle_{n=1}^{\infty}$ satisfies $(\forall n) u^{n} \leq \bar{u}$ and is uniformly bounded from below. Let $s^{n} \rightarrow s^{0}$ and let $b \in \Re_{+}$. Then

$$
\begin{aligned}
& \varlimsup_{n \rightarrow \infty} \int_{S} u^{n} d Q_{s^{n}} \\
\leq & \varlimsup_{n \rightarrow \infty}\left[\int_{S} u^{n} d Q_{s^{n}}-\int_{S}\left(u^{n} \wedge b\right) d Q_{s^{n}}\right]+\varlimsup_{n \rightarrow \infty} \int_{S}\left(u^{n} \wedge b\right) d Q_{s^{n}} \\
\leq & \varlimsup_{n \rightarrow \infty}\left[\int_{S} u^{n} d Q_{s^{n}}-\int_{S}\left(u^{n} \wedge b\right) d Q_{s^{n}}\right]+\int_{S} \varlimsup_{n \rightarrow \infty} u^{n} d Q_{s^{\circ}} \\
= & \varlimsup_{n \rightarrow \infty} \int_{\left\{u^{n} \geq b\right\}}\left(u^{n}-b\right) d Q_{s^{n}}+\int_{S} \varlimsup_{n \rightarrow \infty} u^{n} d Q_{s^{\circ}} \\
\leq & \varlimsup_{\lim _{n \rightarrow \infty}} \int_{\{\bar{u} \geq b\}} \bar{u} d Q_{s^{n}}+\int_{S} \varlimsup_{\lim _{n \rightarrow \infty} u^{n} d Q_{s^{\circ}} .}
\end{aligned}
$$

The second inequality follows from the first paragraph and from the fact that $u^{n} \wedge b \leq b$. The third inequality follows because each $u^{n} \leq \bar{u}$ and because each $u^{n}-b \leq \bar{u}$ since $b \geq 0$. Finally, because this whole inequality holds for all $b \geq 0$, M2 implies the desired inequality.

This paragraph proves the claim when $\left\langle u^{n}\right\rangle_{n=1}^{\infty}$ satisfies $(\forall n) u^{n} \leq \bar{u}$ and completes the proof. Let 
$s^{n} \rightarrow s^{0}$ and let $B \in \Re_{+}$. Then

$$
\begin{aligned}
& \varlimsup_{n \rightarrow \infty} \int_{S} u^{n} d Q_{s^{n}} \\
\leq & \varlimsup_{n \rightarrow \infty} \int_{S}\left(u^{n} \vee(-B)\right) d Q_{s^{n}} \\
\leq & \int_{S}\left(\left(\varlimsup_{n \rightarrow \infty} u^{n}\right) \vee(-B)\right) d Q_{s^{0}} .
\end{aligned}
$$

The second inequality follows from the second paragraph. Since $\left(\varlimsup_{n \rightarrow \infty} u^{n}\right) \vee(-B) \leq \bar{u} \vee 0$ and since $\int_{S}(\bar{u} \vee 0) d Q_{s}<+\infty$ by $\mathrm{M} 2$, the monotone convergence theorem (letting $B \rightarrow+\infty$ ) completes the proof.

Lemma C.2 (U.Q.C. with Densities): Suppose that $Q$ has a density $q: S^{2} \rightarrow \Re_{+}$with respect to a measure $\mu:(\forall s)(\forall E) Q_{s}(E)=\int_{E} q_{s}\left(s^{\prime}\right) \mu\left(d s^{\prime}\right)$. Then $\int \cdot d Q_{s}$ is u.q.c. beneath $\bar{u} \in L(S, T)$ if $(q, \bar{u})$ satisfies:

m1. $\left(\forall\left\langle s^{n}\right\rangle_{n=1}^{\infty} \rightarrow s^{0}\right) \quad q_{s} \rightarrow q_{s} 0$ H-a.e., and

m2. $\int(\bar{u} \vee 0) q_{s} d \mu$ is a real-valued and continuous function of $s$.

Proof: M1 holds by $\mathrm{m} 1$ and Scheffé's theorem (Billingsley, 1986, p. 218). M2 holds because

$$
\begin{aligned}
\left(\forall\left\langle s^{n}\right\rangle_{n=1}^{\infty} \rightarrow s^{0}\right) & \lim _{b \rightarrow \infty} \overline{\lim }_{n \rightarrow \infty} \int_{\{\bar{u} \geq b\}} \bar{u} d Q_{s^{n}} \\
\leq & \lim _{b \rightarrow \infty} \overline{\lim }_{n \rightarrow \infty} \int_{\{\bar{u} \geq b\}}(\bar{u} \vee 0) d Q_{s^{n}} \\
& =\lim _{b \rightarrow \infty} \overline{\lim }_{n \rightarrow \infty} \int_{\{\bar{u} \geq b\}}(\bar{u} \vee 0) q_{s^{n}} d \mu \\
& \leq \lim _{b \rightarrow \infty} \int_{\{\bar{u} \geq b\}}(\bar{u} \vee 0) \overline{\lim }_{n \rightarrow \infty} q_{s^{n}} d \mu \\
& =\lim _{b \rightarrow \infty} \int_{\{\bar{u} \geq b\}}(\bar{u} \vee 0) q_{s^{\circ}} d \mu \\
& =0 .
\end{aligned}
$$

The second inequality holds by Billingsley $(1986, \mathrm{p} .223,16.22)$ because $\left\langle(\bar{u} \vee 0) q_{s^{n}}\right\rangle_{n=1}^{\infty}$ is uniformly integrable by $\mathrm{m} 1, \mathrm{~m} 2$, and Billingsley $(1986, \mathrm{p} .223,16.21)$. The second equality holds by $\mathrm{m} 1$. The final equality holds by $\mathrm{m} 2$ and the dominated convergence theorem.

Lemma C.3 (L.Q.C.): Suppose that $T=\bar{\Re}_{+}$. Then $\int \cdot d Q$, is l.q.c. above 0 if $Q$ satisfies:

$$
\text { M1. }\left(\forall\left(\left.s^{n}\right|_{n=1} ^{\infty} \rightarrow s^{0}\right) \quad \sup _{E \in B_{S}}\left|Q_{s} \approx(E)-Q_{s} \circ(E)\right| \rightarrow 0\right. \text {. }
$$

Proof: As in the first paragraph of Lemma C.1's proof, it may by shown that $\varliminf_{n \rightarrow \infty} \int_{S} u^{n} d Q_{s^{n}} \geq$ $\int_{S} \varliminf_{n \rightarrow \infty} u^{n} d Q_{s}$ for any $\left\langle s^{n}\right\rangle_{n} \rightarrow s^{0}$ and any $\left\langle u^{n}\right\rangle_{n}$ which is uniformly bounded from above. 
Now consider any $\left\langle s^{n}\right\rangle_{n} \rightarrow s^{0}$, any $\left\langle u^{n}\right\rangle_{n}$, and any $B \in \Re_{+}$. By the preceding paragraph

$$
\begin{aligned}
& {\varliminf_{n \rightarrow \infty}}_{n} \int_{S} u^{n} d Q_{s^{n}} \\
\geq & \varliminf_{n \rightarrow \infty} \int_{S} u^{n} \wedge B d Q_{s^{n}} \\
\geq & \int_{S} \underline{\lim }_{n \rightarrow \infty} u^{n} \wedge B d Q_{s^{0}} .
\end{aligned}
$$

The monotone convergence theorem (letting $B \rightarrow+\infty$ ) completes the proof.

Lemma C.4 (L.Q.C. with Densities): Suppose that $T=\bar{\Re}_{+}$, and that $Q$ has a density $q: S^{2} \rightarrow \Re_{+}$with respect to a measure $\mu:(\forall s)(\forall E) Q_{s}(E)=\int_{E} q_{s}\left(s^{\prime}\right) \mu\left(d s^{\prime}\right)$. Then $\int \cdot d Q_{s}$ is l.q.c. above 0 if $q$ satisfies:

$$
\text { m1. }\left(\forall\left\langle s^{n}\right\rangle_{n=1}^{\infty} \rightarrow s^{0}\right) \quad q_{s^{n}} \rightarrow q_{s^{0}} \mu \text {-a.e. }
$$

Proof: M1 holds by $\mathrm{m} 1$ and Scheffé's theorem (Billingsley, 1986, p. 218). Hence l.q.c. follows from Lemma C.3.

\section{Appendix D: Lemmas and Proofs for Section 3 (Nonnegative Preferences)}

Proof D.1 (for Theorem D): Preliminaries. In this paragraph, we show the existence of $d \in \Re_{+}$and $\kappa_{3} \in \Re_{+}$such that

$$
\begin{gathered}
\left(\forall c, m \in \Re_{+}\right)\left(\forall m^{\prime}>m\right) W\left(c, m^{\prime}\right)-W(c, m) \leq \kappa_{3}+d\left(m^{\prime}-m\right), \\
\lambda_{1} \equiv \sup _{s \in S} N_{s} 1<d^{-1}, \\
\lambda_{g} \equiv \sup _{s \in S} \varlimsup_{t \rightarrow \infty}\left[N_{s}^{t}\left(\prod_{q=1}^{t} \gamma_{s_{q}}^{\varepsilon}\right)\right]^{1 / t}<d^{-1}, \text { and } \\
\lambda_{z} \equiv \sup _{s \in S} \varlimsup_{i \rightarrow \infty}\left[N_{s}^{t}\left(\sum_{r=1}^{t}\left(\prod_{q=r+1}^{t} \gamma_{s_{q}}^{\varepsilon}\right) \zeta_{s_{r}}^{\varepsilon}\right)\right]^{1 / t}<d^{-1}
\end{gathered}
$$

If $\delta=\bar{\delta}$, simply set $d=\bar{\delta}$ and $\kappa_{3}=0$. Then (9) is implied by the definition of $\bar{\delta},(10),(11)$, and (12) are implied by $\mathrm{N} 7, \mathrm{~N} 9$, and $\mathrm{N} 11$. If $\delta<\bar{\delta}$, then $\mathrm{N} 7, \mathrm{~N} 9, \mathrm{~N} 11$, and the definition of $\delta$ imply the existence of $d \in \Re_{+}$and $\bar{m} \in \Re_{+}$which satisfy (10), (11), (12), and

$$
(\forall m \geq \bar{m})\left(\forall c \in \Re_{+}\right)\left(\forall m^{\prime}>m\right) W\left(c, m^{\prime}\right)-W(c, m) \leq d\left(m^{\prime}-m\right) .
$$

This inequality implies (9) when $\kappa_{3}$ is set equal to $(\bar{\delta}-d) \bar{m}$ :

$$
\begin{aligned}
& \left(\forall c, m \in \Re_{+}\right)\left(\forall m^{\prime}>m\right) \\
& W\left(c, m^{\prime}\right)-W(c, m)
\end{aligned}
$$




$$
\begin{aligned}
& \leq \begin{cases}\bar{\delta}\left(m^{\prime}-m\right) & \text { if } m^{\prime}-m \leq \bar{m} \\
\bar{\delta} \bar{m}+d\left(m^{\prime}-m-\bar{m}\right) & \text { if } m^{\prime}-m>\bar{m}\end{cases} \\
& = \begin{cases}(\bar{\delta}-d)\left(m^{\prime}-m\right)+d\left(m^{\prime}-m\right) & \text { if } m^{\prime}-m \leq \bar{m} \\
(\bar{\delta}-d) \bar{m}+d\left(m^{\prime}-m\right) & \text { if } m^{\prime}-m>\bar{m}\end{cases} \\
& \leq(\bar{\delta}-d) \bar{m}+d\left(m^{\prime}-m\right) \\
& =\kappa_{3}+d\left(m^{\prime}-m\right) .
\end{aligned}
$$

Define $\kappa_{2} \in \Re_{+}$and $\kappa_{4} \in \Re_{+}$so that

$$
\left(\forall c, m \in \Re_{+}\right) W(c, m) \leq \kappa_{4}+\kappa_{2} c^{\varepsilon}+d m
$$

Such constants exist because

$$
\begin{aligned}
\left(\forall c, m \in \Re_{+}\right) & W(c, m) \\
= & W(c, 0)+(W(c, m)-W(c, 0)) \\
\leq & \kappa_{1}+\kappa_{2} c^{\varepsilon}+(W(c, m)-W(c, 0)) \\
\leq & \kappa_{1}+\kappa_{2} c^{\varepsilon}+\kappa_{3}+d m \\
& =\kappa_{4}+\kappa_{2} c^{\varepsilon}+d m,
\end{aligned}
$$

where the first inequality and the existence of $\kappa_{1}$ and $\kappa_{2}$ are ensured by the definition of $\varepsilon$, the second inequality holds by (9), and the final equality follows by letting $\kappa_{4}$ be $\kappa_{1}+\kappa_{3}$.

For our future convenience, define $H: \Re_{+} \rightarrow L\left(S, \Re_{+}\right)$by $H(y)=\zeta^{\varepsilon}+\gamma^{\varepsilon} y$. Note that

$$
H^{t}(y)=\sum_{r=1}^{t}\left(\prod_{q=r+1}^{t} \gamma_{s_{q}}^{\varepsilon}\right) \zeta_{s_{r}}^{\varepsilon}+\left(\prod_{q=1}^{t} \gamma_{s_{q}}^{\varepsilon}\right) y
$$

and that by the definitions of $\zeta, \gamma$, and $\varepsilon$,

$$
\begin{aligned}
\left(\bar{F}^{t}(y)\right)^{\varepsilon} & \leq\left(\sum_{r=1}^{t}\left(\prod_{q=r+1}^{t} \gamma_{s_{q}}\right) \zeta_{s_{r}}+\left(\prod_{q=1}^{t} \gamma_{s_{q}}\right) y\right)^{\varepsilon} \\
& \leq \sum_{r=1}^{t}\left(\prod_{q=r+1}^{t} \gamma_{s_{q}}^{\varepsilon}\right) \zeta_{s_{r}}^{\varepsilon}+\left(\prod_{q=1}^{t} \gamma_{s_{q}}^{\varepsilon}\right) y^{\varepsilon} \\
& =H^{t}\left(y^{\varepsilon}\right) .
\end{aligned}
$$

This paragraph shows

$$
\begin{aligned}
(\forall s)(\forall y)(\forall t) \leq & W\left(y, M_{s} W\left(\bar{F}(y), M \cdots W\left(\bar{F}^{t}(y), 0\right) \cdots\right)\right) \\
\leq & \kappa_{4}+\kappa_{2} y^{\varepsilon}+d M_{s}\left(\kappa_{4}+\kappa_{2}(\bar{F}(y))^{\varepsilon}+d M\left(\cdots \kappa_{4}+\kappa_{2}\left(\bar{F}^{t}(y)\right)^{\varepsilon} \cdots\right)\right) \\
\leq & \kappa_{4}+\kappa_{2} y^{\varepsilon}+d N_{s}\left(\kappa_{4}+\kappa_{2}(\bar{F}(y))^{\varepsilon}+d M\left(\cdots \kappa_{4}+\kappa_{2}\left(\bar{F}^{t}(y)\right)^{\varepsilon} \cdots\right)\right) \\
& \cdots \\
\leq & \kappa_{4}+\kappa_{2} y^{\varepsilon}+d N_{s}\left(\kappa_{4}+\kappa_{2}(\bar{F}(y))^{\varepsilon}+d N\left(\cdots \kappa_{4}+\kappa_{2}\left(\bar{F}^{t}(y)\right)^{\varepsilon} \cdots\right)\right)
\end{aligned}
$$




$$
\begin{aligned}
& \leq \kappa_{4}+\kappa_{2} y^{\varepsilon}+d N_{s}\left(\kappa_{4}+\kappa_{2} H\left(y^{\varepsilon}\right)+d N\left(\cdots \kappa_{4}+\kappa_{2} H^{t}\left(y^{\varepsilon}\right) \cdots\right)\right) \\
& \leq \sum_{r=0}^{t} d^{r} N_{s}^{r}\left(\kappa_{4}+\kappa_{2} H^{r}\left(y^{\varepsilon}\right)\right) \\
& \leq \kappa_{4} \sum_{r=0}^{t} d^{r} N_{s}^{r} 1+\kappa_{2} \sum_{r=0}^{t} d^{r} N_{s}^{r} H^{r}\left(y^{\varepsilon}\right) \\
& \leq \kappa_{4} \sum_{r=0}^{t} d^{r} \lambda_{1}^{r}+\kappa_{2} \sum_{r=0}^{t} d^{r} N_{s}^{r} H^{r}\left(y^{\varepsilon}\right) \\
& \leq \kappa_{4} \sum_{r=0}^{\infty} d^{r} \lambda_{1}^{r}+\kappa_{2} \sum_{r=0}^{\infty} d^{r} N_{s}^{r} H^{r}\left(y^{\varepsilon}\right) \\
& =\kappa_{4}\left(1-d \lambda_{1}\right)^{-1}+\kappa_{2} \sum_{r=0}^{\infty} d^{r} N_{s}^{r} H^{r}\left(y^{\varepsilon}\right)
\end{aligned}
$$

The first expression is well-defined by $\mathrm{A} 6$ and the fact that $0 \bar{F}(y) \in C$ by Lemma A.1. The first inequality holds by (13), and the second by N4. The successive applications lead to the third inequality. These steps are justified by N3 and the assumption that the $\sigma$-algebra of $\widehat{S}$ is at least as fine as $\mathcal{B}_{S}$. The fourth inequality holds by (15), the fifth and sixth by subadditivity (N5) and subhomogeneity (N6), the seventh by the definition of $\lambda_{1}$, and the eighth by letting $t$ go to infinity. The final equality holds by (10).

In this paragraph, we show that

$$
(\forall s)(\forall y) \quad \sum_{t=0}^{\infty} d^{t} N_{s}^{t} H^{t}\left(y^{\epsilon}\right)<+\infty .
$$

Take any $s$ and $y$. By (11) and (12) we have the existence of a $\hat{d}$ and $\hat{t}$ such that

$$
\begin{gathered}
\hat{d}>d, \\
(\forall t \geq \hat{t})\left[N_{s}^{t}\left(\prod_{q=1}^{t} \gamma_{s_{q}}^{\varepsilon}\right)\right]^{1 / t}<\hat{d}^{-1}, \text { and } \\
(\forall t \geq \hat{t})\left[N_{s}^{t}\left(\sum_{r=1}^{t}\left(\prod_{q=r+1}^{t} \gamma_{s_{q}}^{\varepsilon}\right) \zeta_{s_{r}}^{\varepsilon}\right)\right]^{1 / t}<\hat{d}^{-1}
\end{gathered}
$$

Thus

$$
\begin{aligned}
& \sum_{t=0}^{\infty} d^{t} N_{s}^{t} H^{t}\left(y^{\varepsilon}\right) \\
= & \sum_{t=0}^{\infty} d^{t} N_{s}^{t}\left(\sum_{r=1}^{t}\left(\prod_{q=r+1}^{t} \gamma_{s_{q}}^{\varepsilon}\right) \zeta_{s_{r}}^{\varepsilon}+\left(\prod_{q=1}^{t} \gamma_{s_{q}}^{\varepsilon}\right) y^{\varepsilon}\right) \\
\leq & \sum_{t=0}^{\infty} d^{t} N_{s}^{t}\left(\sum_{r=1}^{t}\left(\prod_{q=r+1}^{t} \gamma_{s_{q}}^{\varepsilon}\right) \zeta_{s_{r}}^{\varepsilon}\right)+y^{\varepsilon} \sum_{t=0}^{\infty} d^{t} N_{s}^{t}\left(\prod_{q=1}^{t} \gamma_{s_{q}}^{\varepsilon}\right) \\
\leq & \sum_{t=0}^{t} d^{t} N_{s}^{t}\left(\sum_{r=1}^{t}\left(\prod_{q=r+1}^{t} \gamma_{s_{q}}^{\varepsilon}\right) \zeta_{s_{r}}^{\varepsilon}\right)+y^{\varepsilon} \sum_{t=0}^{t} d^{t} N_{s}^{t}\left(\prod_{q=1}^{t} \gamma_{s_{q}}^{\varepsilon}\right) \\
& +\sum_{t=\hat{t}+1}^{\infty} d^{t} N_{s}^{t}\left(\sum_{r=1}^{t}\left(\prod_{q=r+1}^{t} \gamma_{s_{q}}^{\varepsilon}\right) \zeta_{s_{r}}^{\varepsilon}\right)+y^{\varepsilon} \sum_{t=i+1}^{\infty} d^{t} N_{s}^{t}\left(\prod_{q=1}^{t} \gamma_{s_{q}}^{\varepsilon}\right) \\
< & \sum_{t=0}^{i} d^{t} N_{s}^{t}\left(\sum_{r=1}^{t}\left(\prod_{q=r+1}^{t} \gamma_{s_{q}}^{\varepsilon}\right) \zeta_{s_{r}}^{\varepsilon}\right)+y^{\varepsilon} \sum_{t=0}^{t} d^{t} N_{s}^{t}\left(\prod_{q=1}^{t} \gamma_{s_{q}}^{\varepsilon}\right)+\sum_{t=\hat{t}+1}^{\infty} d^{t} \hat{d}^{-t}+y^{\varepsilon} \sum_{t=\hat{t}+1}^{\infty} d^{t} \hat{d}^{-t} \\
= & \sum_{t=0}^{i} d^{t} N_{s}^{t}\left(\sum_{r=1}^{t}\left(\prod_{q=r+1}^{t} \gamma_{s_{q}}^{\varepsilon}\right) \zeta_{s_{r}}^{\varepsilon}\right)+y^{\varepsilon} \sum_{t=0}^{t} d^{t} N_{s}^{t}\left(\prod_{q=1}^{t} \gamma_{s_{q}}^{\varepsilon}\right) \\
& +\left(d \hat{d}^{-1}\right)^{\hat{i}+1}\left(1-d \hat{d}^{-1}\right)^{-1}+y^{\varepsilon}\left(d \hat{d}^{-1}\right)^{\hat{t}+1}\left(1-d \hat{d}^{-1}\right)^{-1} \\
< & +\infty
\end{aligned}
$$

The first equality holds by (14), the first inequality holds by subadditivity (N5) and subhomogeneity (N6), the strict inequality holds by (19) and (20), the final equality holds by (18), and the final inequality by $\mathrm{N} 8$ and N10. 
Well-Definition (and Real-Valuedness). Now we can claim

$$
\begin{aligned}
U_{s}(c) & =\lim _{t \rightarrow \infty} W\left(c_{0}, M_{3} W\left(c_{1}, M \cdots W\left(c_{t}, 0\right) \cdots\right)\right) \\
& \leq \lim _{t \rightarrow \infty} W\left(y, M_{s} W\left(\bar{F}(y), M \cdots W\left(\bar{F}^{t}(y), 0\right) \cdots\right)\right) \\
& \leq \kappa_{4}\left(1-d \lambda_{1}\right)+\kappa_{2} \sum_{r=0}^{\infty} d^{r} N_{s}^{r} H^{r}\left(y^{\varepsilon}\right) \\
& <+\infty
\end{aligned}
$$

The equality is the definition of $U$ and the limit of its RHS exists in $\Re_{+} \cup\{+\infty\}$ because the sequence is well-defined by $A 6$ and weakly increasing by $A 1$ and $A 4$. The first inequality follows from $A 1$ and $A 4$, the second from (16), and the final from (10) and (17). Hence the range of $U$ is contained in $\Re_{+}$, as claimed in the definition of $U$.

The finiteness of mean utility $M_{s} U\left({ }_{1} c\right)$ can be similarly derived: modify the above inequality to start with $M_{s} U\left({ }_{1} c\right)$ and modify (16) to start with $M_{s} W\left(\bar{F}(y), M \cdots W\left(\bar{F}^{t}(y), 0\right) \cdots\right)$.

U1, U4-U8. U1 (monotonicity) follows immediately from A1, A4, and the definition of $U$. Then $\mathrm{U} 5, \mathrm{U} 6$, and $\mathrm{U} 7$ are implied by $\mathrm{A} 1, \mathrm{A2}$, and A4. U4 (Koopmans' equation) holds because

$$
\begin{aligned}
& (\forall s)(\forall y)(\forall c \leq 0 \bar{F}(y)) \\
& U_{s}(c) \\
= & \lim _{t \rightarrow \infty} W\left(c_{0}, M_{s} W\left(c_{1}, M \cdots W\left(c_{t}, 0\right) \cdots\right)\right) \\
= & W\left(c_{0}, \lim _{t \rightarrow \infty} M_{s} W\left(c_{1}, M \cdots W\left(c_{t}, 0\right) \cdots\right)\right) \\
= & W\left(c_{0}, M_{s} \lim _{t \rightarrow \infty} W\left(c_{1}, M \cdots W\left(c_{t}, 0\right) \cdots\right)\right) \\
= & W\left(c_{0}, M_{s} U\left({ }_{1} c\right)\right) .
\end{aligned}
$$

These four equalities hold by the definition of $U, \mathbf{A 3}, \mathbf{A 5}$, and the definition of $U$ again. U8 holds because

$$
\begin{aligned}
(\forall s)\left(\forall y_{-}\right) & J_{s}^{+} \circ \bar{F}_{s}\left(y_{-}\right) \\
= & U_{s}\left({ }_{0} \bar{F}\left(\bar{F}_{s}\left(y_{-}\right)\right)\right) \\
= & \lim _{t \rightarrow \infty} W\left(\bar{F}_{s}\left(y_{-}\right), M_{s} W\left(\bar{F}^{2}\left(y_{-}\right), M \cdots W\left(\bar{F}^{t}\left(y_{-}\right), 0\right) \cdots\right)\right) \\
\leq & \kappa_{4}\left(1-d \lambda_{1}\right)^{-1}+\kappa_{2} \sum_{t=0}^{\infty} d^{t} N_{s}^{t} H^{t}\left(\left(\bar{F}_{s}\left(y_{-}\right)\right)^{\varepsilon}\right) \\
\leq & \kappa_{4}\left(1-d \lambda_{1}\right)^{-1}+\kappa_{2} \sum_{t=0}^{\infty} d^{t} N_{s}^{t}\left[\sum_{r=1}^{t}\left(\prod_{q=r+1}^{t} \gamma_{s_{q}}^{\varepsilon}\right) \zeta_{s_{r}}^{\varepsilon}+\left(\prod_{q=1}^{t} \gamma_{s_{q}}^{\varepsilon}\right)\left(\zeta_{s}^{\varepsilon}+\gamma_{s}^{\varepsilon} y_{-}^{\varepsilon}\right)\right]
\end{aligned}
$$

by (16), (14), (15) (at $t=1$ ), and because $M$ is u.q.c. beneath the RHS of this inequality by N5 and N12.

U2 and U3 (Biconvergence). Take any $s, y$, and $c$ such that $c \leq_{0} \bar{F}(y)$. Take any $\xi>0$. Let $i$ be such that

$$
\left(\bar{\delta} \lambda_{1}\right)^{i} \frac{\kappa_{3}}{1-d \lambda_{1}}<\xi
$$

Such an $i$ exists because the RHS is positive and because the limit of the LHS is 0 by N7. Let $j$ be such that

$$
(\forall t \geq j) \quad d^{t-i} N_{s}^{t} U\left({ }_{t} \bar{F}(y)\right)<\bar{\delta}^{-i} \xi-\frac{\kappa_{3} \lambda_{1}^{i}}{1-d \lambda_{1}} .
$$


Such a $j$ exists because the RHS is positive (by the definition of $i$ ) and because the limit of the LHS is 0 by the remainder of this paragraph. First, note that

$$
\begin{aligned}
& d^{t} N_{s}^{t} U\left({ }_{t} \bar{F}(y)\right) \\
= & d^{t} N_{s}^{t} \lim _{q \rightarrow \infty} W\left(\bar{F}^{t}(y), M_{s} W\left(\bar{F}^{t+1}(y), M \cdots W\left(\bar{F}^{q}(y), 0\right) \cdots\right)\right) \\
\leq & d^{t} N_{s}^{t}\left(\kappa_{4}\left(1-d \lambda_{1}\right)^{-1}+\kappa_{2} \sum_{r=0}^{\infty} d^{r} N^{r} H^{r}\left(\left(\bar{F}^{t}(y)\right)\right)\right) \\
\leq & d^{t} N_{s}^{t}\left(\kappa_{4}\left(1-d \lambda_{1}\right)^{-1}+\kappa_{2} \sum_{r=0}^{\infty} d^{r} N^{r} H^{t+r}\left(y^{\varepsilon}\right)\right) \\
\leq & \kappa_{4} d^{t} N_{s}^{t}\left(1-d \lambda_{1}\right)^{-1}+\kappa_{2} d^{t} N_{s}^{t} \sum_{r=0}^{\infty} d^{r} N^{r} H^{t+r}\left(y^{\varepsilon}\right) \\
\leq & \kappa_{4}\left(d \lambda_{1}\right)^{t}\left(1-d \lambda_{1}\right)^{-1}+\kappa_{2} d^{t} N_{s}^{t} \sum_{r=0}^{\infty} d^{r} N^{r} H^{t+r}\left(y^{\varepsilon}\right) \\
\leq & \kappa_{4}\left(d \lambda_{1}\right)^{t}\left(1-d \lambda_{1}\right)^{-1}+\kappa_{2} \sum_{q=t}^{\infty} d^{q} N_{s}^{q} H^{q}\left(y^{\varepsilon}\right) .
\end{aligned}
$$

The opening equality is the definition of $U$. The first inequality holds by (16), the second by (15), the third by subadditivity (N5) and subhomogeneity (N6), the fourth by subhomogeneity (N6) and the definition of $\lambda_{1}$, and the last by monotone convergence (N2), subadditivity (N5), and subhomogeneity (N6). Thus we conclude

$$
\begin{aligned}
& \lim _{t \rightarrow \infty} d^{t-i} N_{s}^{t} U\left({ }_{t} \bar{F}(y)\right) \\
= & d^{-i} \lim _{t \rightarrow \infty} d^{t} N_{s}^{t} U\left({ }_{t} \bar{F}(y)\right) \\
\leq & d^{-i} \lim _{t \rightarrow \infty}\left(\kappa_{4}\left(d \lambda_{1}\right)^{t}\left(1-d \lambda_{1}\right)^{-1}+\kappa_{2} \sum_{r=t}^{\infty} d^{r} N_{s}^{r} H^{r}\left(y^{\varepsilon}\right)\right) \\
= & 0 .
\end{aligned}
$$

The inequality follows from (21) and the final equality follows from (10) and (17).

Take any $t \geq j$.

This paragraph shows by backward induction on $r$ that

$$
\begin{aligned}
& (\forall r \in\{i, \ldots, t-1\}) \\
& N_{s}^{r}\left[W\left(c_{r}, M \cdots W\left(c_{t-1}, M U\left({ }_{t} \bar{F}(y)\right)\right) \cdots\right)-W\left(c_{r}, M \cdots W\left(c_{t-1}, 0\right) \cdots\right)\right] \\
\leq & \kappa_{3} \lambda_{1}^{r}\left(1-d \lambda_{1}\right)^{-1}+d^{t-r} N_{s}^{t} U\left({ }_{t} \bar{F}(y)\right) .
\end{aligned}
$$

The initial step $(r=t-1)$ holds because

$$
\begin{aligned}
& N_{s}^{t-1}\left[W\left(c_{t-1}, M U\left({ }_{t} \bar{F}(y)\right)\right)-W\left(c_{t-1}, 0\right)\right] \\
\leq & N_{s}^{t-1}\left[\kappa_{3}+d M U\left({ }_{t} \bar{F}(y)\right)\right] \\
\leq & N_{s}^{t-1}\left[\kappa_{3}+d N U\left({ }_{t} \bar{F}(y)\right)\right] \\
\leq & \kappa_{3} N_{s}^{t-1} 1+d N_{s}^{t} U\left({ }_{t} \bar{F}(y)\right) \\
\leq & \kappa_{3} \lambda_{1}^{t-1}+d N_{s}^{t} U\left({ }_{t} \bar{F}(y)\right) \\
\leq & \kappa_{3} \lambda_{1}^{t-1}\left(1-d \lambda_{1}\right)^{-1}+d N_{s}^{t} U\left({ }_{t} \bar{F}(y)\right) .
\end{aligned}
$$


The first inequality holds by (9), the second by N4, the third by subadditivity (N5) and subhomogeneity (N6), the fourth by the definition of $\lambda_{1}$, and the fifth by (10). The inductive steps follow by

$$
\begin{aligned}
& N_{s}^{r}\left[W\left(c_{r}, M \cdots W\left(c_{t-1}, M U\left({ }_{t} \bar{F}(y)\right)\right) \cdots\right)-W\left(c_{r}, M \cdots W\left(c_{t-1}, 0\right) \cdots\right)\right] \\
\leq & N_{s}^{r}\left(\kappa_{3}+d\left[M W\left(c_{r+1}, M \cdots W\left(c_{t-1}, M U\left({ }_{t} \bar{F}(y)\right)\right) \cdots\right)-M W\left(c_{r+1}, M \cdots W\left(c_{t-1}, 0\right) \cdots\right)\right]\right) \\
\leq & N_{s}^{r}\left(\kappa_{3}+d N\left[W\left(c_{r+1}, M \cdots W\left(c_{t-1}, M U\left({ }_{t} \bar{F}(y)\right)\right) \cdots\right)-W\left(c_{r+1}, M \cdots W\left(c_{t-1}, 0\right) \cdots\right)\right]\right) \\
\leq & \kappa_{3} N_{s}^{r} 1+d N_{s}^{r+1}\left[W\left(c_{r+1}, M \cdots W\left(c_{t-1}, M U\left({ }_{t} \bar{F}(y)\right)\right) \cdots\right)-W\left(c_{r+1}, M \cdots W\left(c_{t-1}, 0\right) \cdots\right)\right] \\
\leq & \kappa_{3} \lambda_{1}^{r}+d\left[\kappa_{3} \lambda_{1}^{r+1}\left(1-d \lambda_{1}\right)^{-1}+d^{t-(r+1)} N_{s}^{t} U\left({ }_{t} \bar{F}(y)\right)\right] \\
= & \kappa_{3} \lambda_{1}^{r}\left(1-d \lambda_{1}\right)^{-1}+d^{t-r} N_{s}^{t} U\left({ }_{t} \bar{F}(y)\right) .
\end{aligned}
$$

The first inequality holds by (9), the second by N4, the third by subadditivity (N5) and subhomogeneity (N6), and the fourth by the definition of $\lambda_{1}$ and the inductive hypothesis. The final equality is simple algebra.

Hence we may conclude that

$$
\begin{aligned}
& N_{s}^{i}\left[W\left(c_{i}, M \cdots W\left(c_{t-1}, M U\left({ }_{t} \bar{F}(y)\right)\right) \cdots\right)-W\left(c_{i}, M \cdots W\left(c_{t-1}, 0\right) \cdots\right)\right] \\
\leq & \kappa_{3} \lambda_{1}^{i}\left(1-d \lambda_{1}\right)^{-1}+d^{t-i} N_{s}^{t} U\left({ }_{t} \bar{F}(y)\right) \\
\leq & \kappa_{3} \lambda_{1}^{i}\left(1-d \lambda_{1}\right)^{-1}+\bar{\delta}^{-i} \xi-\kappa_{3} \lambda_{1}^{i}\left(1-d \lambda_{1}\right)^{-1} \\
\leq & \delta^{-i} \xi .
\end{aligned}
$$

The first inequality is (22) at $r=i$ and the second holds by the definition of $j$ and the fact that $t \geq j$.

This paragraph shows by backward induction on $r$ that

$$
\begin{gathered}
(\forall r \in\{0,1, \ldots, i\}) \\
N_{s}^{r}\left[W\left(c_{r}, M \cdots W\left(c_{t-1}, M U\left({ }_{t} \bar{F}(y)\right)\right) \cdots\right)-W\left(c_{r}, M \cdots W\left(c_{t-1}, 0\right) \cdots\right)\right] \leq \bar{\delta}^{-r} \xi,
\end{gathered}
$$

where $N_{s}^{1}=N_{s}$ and $N_{s}^{0}$ is the identity operator. The initial step $(r=i)$ is identical to (23). The inductive step holds because

$$
\begin{aligned}
& N_{s}^{r}\left[W\left(c_{r}, M \cdots W\left(c_{t-1}, M U\left({ }_{t} \bar{F}(y)\right)\right) \cdots\right)-W\left(c_{r}, M \cdots W\left(c_{t-1}, 0\right) \cdots\right)\right] \\
\leq & N_{s}^{r}\left(\bar{\delta}\left[M W\left(c_{r+1}, M \cdots W\left(c_{t-1}, M U\left({ }_{t} \bar{F}(y)\right)\right) \cdots\right)-M W\left(c_{r+1}, M \cdots W\left(c_{t-1}, 0\right) \cdots\right)\right]\right) \\
\leq & N_{s}^{r}\left(\bar{\delta}\left(N\left[W\left(c_{r+1}, M \cdots W\left(c_{t-1}, M U\left({ }_{t} \bar{F}(y)\right)\right) \cdots\right)-W\left(c_{r+1}, M \cdots W\left(c_{t-1}, 0\right) \cdots\right)\right]\right)\right) \\
\leq & \bar{\delta} N_{s}^{r+1}\left[W\left(c_{r+1}, M \cdots W\left(c_{t-1}, M U\left({ }_{t} \bar{F}(y)\right)\right) \cdots\right)-W\left(c_{r+1}, M \cdots W\left(c_{t-1}, 0\right) \cdots\right)\right] \\
\leq & \bar{\delta}^{-(r+1)} \xi \\
= & \bar{\delta}^{-r} \xi .
\end{aligned}
$$

The first inequality holds by the definition of $\bar{\delta}$, the second by N4, the third by subhomogeneity (N6), and the fourth by the inductive hypothesis.

We conclude that

$$
U\left({ }_{0} c_{t-1},{ }_{t} \bar{F}(y)\right)-U\left(0 c_{t-1},{ }_{t} 0\right)
$$




$$
\begin{aligned}
& =W\left(c_{0}, M \cdots W\left(c_{t-1}, M U\left({ }_{t} \bar{F}(y)\right)\right) \cdots\right)-W\left(c_{0}, M \cdots W\left(c_{t-1}, M U\left({ }_{t} 0\right)\right) \cdots\right) \\
& \leq W\left(c_{0}, M \cdots W\left(c_{t-1}, M U\left({ }_{t} \bar{F}(y)\right)\right) \cdots\right)-W\left(c_{0}, M \cdots W\left(c_{t-1}, 0\right) \cdots\right) \\
& \leq \xi
\end{aligned}
$$

The equality follows from Koopmans' equation, the first inequality holds because the range of $M$ is nonnegative, and the final inequality holds by (24) at $r=0$. Since this inequality has been derived for any $t \geq j$, our proof of biconvergence is complete.

Proof D.2 (for Proposition 1): Note that

$$
\begin{aligned}
(\forall s)(\forall t) & N_{s}^{t}\left(\sum_{r=1}^{t}\left(\prod_{q=r+1}^{t} \gamma_{s_{q}}^{\varepsilon}\right) \zeta_{s_{r}}^{\varepsilon}\right) \\
\leq & N_{s}^{t}\left(\sum_{r=1}^{t}\left(\prod_{q=r+1}^{t} \gamma_{s_{q}}^{\varepsilon}\right) k^{-\varepsilon} \gamma_{s_{r}}^{\varepsilon}\right) \\
\leq & N_{s}^{t}\left(k^{-\varepsilon} \sum_{r=1}^{t}\left(\prod_{q=1}^{t} \gamma_{s_{q}}^{\varepsilon}\right)\right) \\
= & N_{s}^{t}\left(k^{-\varepsilon^{2}} t\left(\prod_{q=1}^{t} \gamma_{s_{q}}^{\varepsilon}\right)\right) \\
\leq & k^{-\varepsilon} t N_{s}^{t}\left(\prod_{q=1}^{t} \gamma_{s_{q}}^{\varepsilon}\right)
\end{aligned}
$$

The first inequality holds by $\zeta \leq k^{-1} \gamma$, the second by $\gamma \geq 1$, and the third by N6. Hence

$$
\begin{aligned}
& \lambda_{z}=\sup _{s \in S} \varlimsup_{\lim _{t \rightarrow \infty}}\left[N_{s}^{t}\left(\sum_{r=1}^{t}\left(\prod_{q=r+1}^{t} \gamma_{s_{q}}^{\varepsilon}\right) \zeta_{s_{r}}^{\varepsilon}\right)\right]^{1 / t} \\
& \leq \sup _{s \in S} \varlimsup_{\lim _{t \rightarrow \infty}}\left[k^{-\varepsilon} t N_{s}^{t}\left(\prod_{q=1}^{t} \gamma_{s_{q}}^{\varepsilon}\right)\right]^{1 / t} \\
& =\sup _{s \in S} \varlimsup_{\lim _{t \rightarrow \infty}}\left[k^{-\varepsilon} t\right]^{1 / t}\left[N_{s}^{t}\left(\prod_{q=1}^{t} \gamma_{s_{q}}^{\varepsilon}\right)\right]^{1 / t} \\
& =\lambda_{g} \text {. }
\end{aligned}
$$

Furthermore,

$$
\begin{aligned}
& \left(\forall k_{1}, k_{2}\right)\left(\forall d<\left(\lambda_{g} \vee \lambda_{z}\right)^{-1}\right)\left(\forall y_{-}\right) \\
& k_{1}+k_{2}\left[\sum_{t=0}^{\infty} d^{t} N_{s}^{t}\left(\sum_{r=1}^{t}\left(\prod_{q=r+1}^{t} \gamma_{s_{q}}^{\varepsilon}\right) \zeta_{s}^{\varepsilon}\right)+\sum_{t=0}^{\infty} d^{t} N_{s}^{t}\left(\prod_{q=1}^{t} \gamma_{s_{q}}^{\varepsilon}\right)\left(\zeta_{s}^{\varepsilon}+\gamma_{s}^{\varepsilon} y_{-}^{\varepsilon}\right)\right] \\
\leq & k_{1}+k_{2}\left[\sum_{t=0}^{\infty} d^{t} k^{-\varepsilon} t N_{s}^{t}\left(\prod_{q=1}^{t} \gamma_{s_{q}}^{\varepsilon}\right)+\sum_{t=0}^{\infty} d^{t} N_{s}^{t}\left(\prod_{q=1}^{t} \gamma_{s_{q}}^{\varepsilon}\right)\left(\gamma_{s}^{\varepsilon}\left(k^{-\varepsilon}+y_{-}^{\varepsilon}\right)\right)\right] \\
\leq & k_{1}+k_{2}\left[k^{-\varepsilon} \sum_{t=0}^{\infty} d^{t} t N_{s}^{t}\left(\prod_{q=1}^{t} \gamma_{s_{q}}^{\varepsilon}\right)+\gamma_{s}^{\varepsilon}\left(k^{-\varepsilon}+y_{-}^{\varepsilon}\right) \sum_{t=0}^{\infty} d^{t} N_{s}^{t}\left(\prod_{q=1}^{t} \gamma_{s_{q}}^{\varepsilon}\right)\right] \\
\leq & k_{1}+k_{2}\left[\left(k^{-\varepsilon}+y_{-}^{\varepsilon}\right) \gamma_{s}^{\varepsilon} \sum_{t=0}^{\infty} d^{t}(t+1) N_{s}^{t}\left(\prod_{q=1}^{t} \gamma_{s_{q}}^{\varepsilon}\right)\right] . \square
\end{aligned}
$$

Lemma D.3: Suppose $\varepsilon>0$ and $\alpha>0$. In each of the three technologies, define $N=\left(E(\cdot)^{\alpha}\right)^{1 / \alpha}$ and $(\forall s) \gamma_{s}=F_{s}^{\prime}(0)$ (recall $F$ is linear). (a) Then 


$$
\begin{array}{ll}
\lambda_{g}=\exp \left(\frac{\alpha}{2}\left(\frac{\varepsilon \sigma}{1-b}\right)^{2}\right) & \text { in Technology 1, } \\
\lambda_{g}=\left(\frac{1-b}{2 \alpha \varepsilon r}\right)^{1 / \alpha}\left(\exp \left(\frac{\alpha \varepsilon r}{1-b}\right)-\exp \left(-\frac{\alpha \varepsilon r}{1-b}\right)\right)^{1 / \alpha} & \text { in Technology 2, and } \\
\lambda_{g}=\left(\frac{\Gamma\left(\frac{\alpha \varepsilon}{1-b}+r\right)}{\Gamma(r)}\right)^{1 / \alpha} & \text { in Technology 9. }
\end{array}
$$

(b) Furthermore, $\left(E(\cdot)^{\alpha}\right)^{1 / \alpha}$ is u.q.c. beneath $\bar{u}$ in any of these three cases, when $\bar{u}$ has been defined by

$$
\bar{u}_{s}=\kappa_{5}+\kappa_{6} \gamma_{s}^{\epsilon} \sum_{r=0}^{\infty} d^{r} N_{s}^{r}\left(\prod_{q=1}^{r} \gamma_{s_{q}}^{\varepsilon}\right)
$$

for any $\kappa_{5}, \kappa_{6} \in \Re_{+}$and any $d \in\left(0, \lambda_{g}^{-1}\right)$.

Proof: Technology 1 (Lognormal). This paragraph shows by induction on $k$ that

$$
(\forall s)(\forall k \geq 1) \quad N_{s}^{k}\left(\prod_{q=1}^{k} \gamma_{s_{q}}^{\varepsilon}\right)=\exp \left(\sum_{i=1}^{k} b^{i} \varepsilon s+\frac{1}{2} \sum_{i=1}^{k}\left(\sum_{j=0}^{i-1} b^{j}\right)^{2} \alpha \varepsilon^{2} \sigma^{2}\right)
$$

Recall that

$$
(\forall c \in \Re) \quad E_{s} \exp \left(c s^{\prime}\right)=\exp \left(c b s+\frac{1}{2} c^{2} \sigma^{2}\right) .
$$

by Mood, Graybill, and Boes (1974, p. 541). The initial step $(k=1)$ follows immediately from (26):

$$
\begin{aligned}
N_{s}\left(\gamma_{s_{1}}^{\varepsilon}\right) & =\left(E_{s} \exp \left(\alpha \varepsilon s_{1}\right)\right)^{1 / \alpha} \\
& =\exp \left(b \varepsilon s+\frac{1}{2} \alpha \varepsilon^{2} \sigma^{2}\right)
\end{aligned}
$$

The following steps $(k \geq 2)$ hold as follows:

$$
\begin{aligned}
& N_{s}^{k}\left(\prod_{q=1}^{k} \gamma_{s_{q}}^{\varepsilon}\right) \\
= & N_{s}\left(\gamma_{s_{1}}^{\varepsilon} N_{s_{1}}^{k-1}\left(\prod_{q=2}^{k} \gamma_{s_{q}}^{\varepsilon}\right)\right) \\
= & N_{s}\left(\gamma_{s_{1}}^{\varepsilon} \exp \left(\sum_{i=1}^{k-1} b^{i} \varepsilon s_{1}+\frac{1}{2} \sum_{i=1}^{k-1}\left(\sum_{j=0}^{i-1} b^{j}\right)^{2} \alpha \varepsilon^{2} \sigma^{2}\right)\right) \\
= & N_{s}\left(\exp \left(\sum_{i=0}^{k-1} b^{i} \varepsilon s_{1}+\frac{1}{2} \sum_{i=1}^{k-1}\left(\sum_{j=0}^{i-1} b^{j}\right)^{2} \alpha \varepsilon^{2} \sigma^{2}\right)\right) \\
= & \left(E_{s} \exp \left(\sum_{i=0}^{k-1} b^{i} \alpha \varepsilon s_{1}+\frac{1}{2} \sum_{i=1}^{k-1}\left(\sum_{j=0}^{i-1} b^{j}\right)^{2} \alpha^{2} \varepsilon^{2} \sigma^{2}\right)\right)^{1 / \alpha} \\
= & \left(\exp \left(\sum_{i=1}^{k} b^{i} \alpha \varepsilon s+\frac{1}{2} \sum_{i=1}^{k-1}\left(\sum_{j=0}^{i-1} b^{j}\right)^{2} \alpha^{2} \varepsilon^{2} \sigma^{2}+\frac{1}{2}\left(\sum_{i=0}^{k-1} b^{i}\right)^{2} \alpha^{2} \varepsilon^{2} \sigma^{2}\right)\right)^{1 / \alpha} \\
= & \exp \left(\sum_{i=1}^{k} b^{i} \varepsilon s+\frac{1}{2} \sum_{i=1}^{k}\left(\sum_{j=0}^{i-1} b^{j}\right)^{2} \alpha \varepsilon^{2} \sigma^{2}\right)
\end{aligned}
$$

where the first five steps hold by: homogeneity, the induction hypothesis, $\gamma_{s}=e^{s}$, the definition of $N(=M)$, and (26). 
(25) allows us to conclude

$$
\begin{aligned}
\lambda_{g} & =\sup _{s \in S} \lim _{k \rightarrow \infty}\left(N_{s}^{k}\left(\prod_{q=1}^{k} \gamma_{s_{q}}^{\varepsilon}\right)\right)^{1 / k} \\
& =\sup _{s \in S} \lim _{k \rightarrow \infty}\left(\exp \left(\sum_{i=1}^{k} b^{i} \varepsilon s+\frac{1}{2} \sum_{i=1}^{k}\left(\sum_{j=0}^{i-1} b^{j}\right)^{2} \alpha \varepsilon^{2} \sigma^{2}\right)\right)^{1 / k} \\
& =\sup _{s \in S} \lim _{k \rightarrow \infty} \exp \left(\frac{1}{k} \sum_{i=1}^{k} b^{i} \varepsilon s+\frac{1}{2 k} \sum_{i=1}^{k}\left(\sum_{j=0}^{i-1} b^{j}\right)^{2} \alpha \varepsilon^{2} \sigma^{2}\right) \\
& =\exp \left(\frac{\alpha}{2}\left(\frac{\varepsilon \sigma}{1-b}\right)^{2}\right)
\end{aligned}
$$

because $|b|<1$.

(25) also allows us to conclude

$$
\begin{aligned}
(\forall s)\left(\forall \kappa_{5}, \kappa_{6}\right) \\
\left(\bar{u}_{s}\right)^{\alpha}=\left[\kappa_{5}+\kappa_{6} \gamma_{s}^{\varepsilon} \sum_{r=0}^{\infty} d^{r} N_{s}^{r}\left(\prod_{q=1}^{r} \gamma_{s_{q}}^{\varepsilon}\right)\right]^{\alpha} \\
=\left[\kappa_{5}+\kappa_{6} \gamma_{s}^{\varepsilon}\left(1+\sum_{r=1}^{\infty} d^{r} N_{s}^{r}\left(\prod_{q=1}^{r} \gamma_{s_{q}}^{\varepsilon}\right)\right)\right]^{\alpha} \\
=\left[\kappa_{5}+\kappa_{6} \gamma_{s}^{\varepsilon}\left(1+\sum_{r=1}^{\infty} d^{r} \exp \left(\sum_{i=1}^{r} b^{i} \varepsilon s+\frac{1}{2} \sum_{i=1}^{r}\left(\sum_{j=0}^{i-1} b^{j}\right)^{2} \alpha \varepsilon^{2} \sigma^{2}\right)\right)\right]^{\alpha} \\
=\left[\kappa_{5}+\kappa_{6} \gamma_{s}^{\varepsilon}\left(1+\sum_{r=1}^{\infty} d^{r} \exp \left(\sum_{i=1}^{r} b^{i} \varepsilon s\right) \exp \left(\frac{1}{2} \sum_{i=1}^{r}\left(\sum_{j=0}^{i-1} b^{j}\right)^{2} \alpha \varepsilon^{2} \sigma^{2}\right)\right)\right]^{\alpha} \\
\leq\left[\kappa_{5}+\kappa_{6} \gamma_{s}^{\varepsilon}\left(1+\sum_{r=1}^{\infty} d^{r}\left(1+\exp \left(\frac{2 b \varepsilon s}{1-b}\right)\right) \exp \left(\frac{1}{2} \sum_{i=1}^{r}\left(\sum_{j=0}^{i-1} b^{j}\right)^{2} \alpha \varepsilon^{2} \sigma^{2}\right)\right)\right]^{\alpha} \\
=\left[\kappa_{5}+\kappa_{6} \gamma_{s}^{\varepsilon}\left(1+\left(1+\exp \left(\frac{2 b \varepsilon s}{1-b}\right)\right) \sum_{r=1}^{\infty} d^{r} \exp \left(\frac{1}{2} \sum_{i=1}^{r}\left(\sum_{j=0}^{i-1} b^{j}\right)^{2} \alpha \varepsilon^{2} \sigma^{2}\right)\right)\right]^{\alpha} \\
=\left[\kappa_{5}+\kappa_{6} \gamma_{s}^{\varepsilon}\left(1+\left(1+\exp \left(\frac{2 b \varepsilon s}{1-b}\right)\right) \sum_{r=1}^{\infty}\left[d \exp \left(\frac{1}{2 r} \sum_{i=1}^{r}\left(\sum_{j=0}^{i-1} b^{j}\right)^{2} \alpha \varepsilon^{2} \sigma^{2}\right)\right]^{r}\right)\right]^{\alpha} \\
=\left[\kappa_{5}+\kappa_{6} \gamma_{s}^{\varepsilon}\left(1+\left(1+\exp \left(\frac{2 b \varepsilon s}{1-b}\right)\right) \kappa_{7}\right)\right]^{\alpha} \\
=\left[\kappa_{5}+\kappa_{8} \gamma_{s}^{\varepsilon}+\kappa_{9} \exp \left(\frac{2 b \varepsilon s}{1-b}\right) \gamma_{s}^{\varepsilon}\right]^{\alpha} \\
=\left[\kappa_{5}+\kappa_{8} \exp (\varepsilon s)+\kappa_{9} \exp \left(\frac{2 b \varepsilon s}{1-b}\right) \exp (\varepsilon s)\right]^{\alpha} \\
=\left[\kappa_{5}+\kappa_{8} \exp (\varepsilon s)+\kappa_{9} \exp \left(\frac{1+b}{1-b} \varepsilon s\right)\right]^{\alpha} \\
\leq \kappa_{10}+\kappa_{11} \exp \left(\kappa_{12} s\right)+\kappa_{13} \exp \left(\kappa_{14} s\right) \\
=\left(3 \kappa_{5}+\left(3 \kappa_{8}\right)^{\alpha} \exp (\alpha \varepsilon s)+\left(3 \kappa_{9}\right)^{\alpha} \exp \left(\frac{1+b}{1-b} \alpha \varepsilon s\right)\right. \\
= \\
= \\
=
\end{aligned}
$$

where every $\kappa$ is a nonnegative real. The third equality holds by (25), and the first inequality is justified by

$$
(\forall b \in(-1,1))(\forall r)(\forall s) \quad \exp \left(\sum_{i=1}^{r} b^{i} \varepsilon s\right)
$$




$$
\begin{aligned}
& \leq\left\{\begin{array}{lll}
\exp \left(\frac{b \varepsilon s}{1-b}\right) & \text { if } & b>0 \text { and } s>0 \\
1 & \text { if } & b s \leq 0 \\
\exp (b \varepsilon s) & \text { if } & b<0 \text { and } s<0
\end{array}\right. \\
& \leq\left(1+\exp \left(\frac{2 b \varepsilon s}{1-b}\right)\right) .
\end{aligned}
$$

$\kappa_{7}$ is finite because the limit (as $r \rightarrow \infty$ ) of the inside of the brackets is less than 1 since $d<\lambda_{g}^{-1}$. The second inequality holds because of the 3 's.

Lemma C.2 shows that $E_{s}$ is u.q.c. beneath the RHS of (27): $\mathrm{m} 1$ is obvious and $\mathrm{m} 2$ holds because

$$
\begin{aligned}
& E_{s}\left(\kappa_{10}+\kappa_{11} \exp \left(\kappa_{12} s^{\prime}\right)+\kappa_{13} \exp \left(\kappa_{14} s^{\prime}\right)\right) \\
= & \kappa_{10}+\kappa_{11} \exp \left(\kappa_{12} b s+\frac{1}{2} \kappa_{12}^{2} \sigma^{2}\right)+\kappa_{13} \exp \left(\kappa_{14} b s+\frac{1}{2} \kappa_{14}^{2} \sigma^{2}\right)
\end{aligned}
$$

by (26). Hence (27) shows that $E_{s}$ is u.q.c. beneath $\bar{u}^{\alpha}$. Hence $\left(E_{s}(\cdot)^{\alpha}\right)^{1 / \alpha}$ is u.q.c. beneath $\bar{u}$ by the monotonic invariance of u.q.c. (see U8 within Proof of Lemma B.1).

Technology 2 (Loguniform). This paragraph shows by induction on $k$ that

$$
(\forall s)(\forall k \geq 1) \quad N_{s}^{k}\left(\prod_{q=1}^{k} \gamma_{s_{q}}^{\varepsilon}\right)=\prod_{i=1}^{k}\left(2 A_{i}\right)^{-1 / \alpha} \prod_{i=1}^{k}\left(\exp \left(A_{i}\right)-\exp \left(-A_{i}\right)\right)^{1 / \alpha} \cdot \exp \left(\sum_{i=1}^{k} b^{i} \varepsilon s\right)
$$

where

$$
A_{i} \equiv r \sum_{j=0}^{i-1} b^{j} \alpha \varepsilon
$$

The initial step $(k=1)$ is immediate:

$$
\begin{aligned}
N_{s}\left(\gamma_{s_{1}}^{\varepsilon}\right) & =\left(\int_{b s-r}^{b s+r} \exp \left(\alpha \varepsilon s_{1}\right) \frac{1}{2 r} d s_{1}\right)^{1 / \alpha} \\
& =\left(\frac{1}{2 \alpha \varepsilon r}\right)^{1 / \alpha}(\exp (\alpha \varepsilon r)-\exp (-\alpha \varepsilon r))^{1 / \alpha} \exp (b \varepsilon s) .
\end{aligned}
$$

The following steps $(k \geq 2)$ hold as follows:

$$
\begin{aligned}
& N_{s}^{k}\left(\prod_{q=1}^{k} \gamma_{s_{q}}^{\varepsilon}\right) \\
= & N_{s}\left(\gamma_{s_{1}}^{\varepsilon} N_{s_{1}}^{k-1}\left(\prod_{q=2}^{k} \gamma_{s_{q}}^{\varepsilon}\right)\right) \\
= & N_{s}\left(\gamma_{s_{1}}^{\varepsilon} \prod_{i=1}^{k-1}\left(2 A_{i}\right)^{-1 / \alpha} \prod_{i=1}^{k-1}\left(\exp \left(A_{i}\right)-\exp \left(-A_{i}\right)\right)^{1 / \alpha} \cdot \exp \left(\sum_{i=1}^{k-1} b^{i} \varepsilon s_{1}\right)\right) \\
= & N_{s}\left(\prod_{i=1}^{k-1}\left(2 A_{i}\right)^{-1 / \alpha} \prod_{i=1}^{k-1}\left(\exp \left(A_{i}\right)-\exp \left(-A_{i}\right)\right)^{1 / \alpha} \cdot \exp \left(\sum_{i=0}^{k-1} b^{i} \varepsilon s_{1}\right)\right) \\
= & \left(\int_{b s-r}^{b s+r} \prod_{i=1}^{k-1}\left(2 A_{i}\right)^{-1} \prod_{i=1}^{k-1}\left(\exp \left(A_{i}\right)-\exp \left(-A_{i}\right)\right) \cdot \exp \left(\sum_{i=0}^{k-1} b^{i} \alpha \varepsilon s_{1}\right) \frac{1}{2 r} d s_{1}\right)^{1 / \alpha} \\
= & \prod_{i=1}^{k-1}\left(2 A_{i}\right)^{-1 / \alpha} \prod_{i=1}^{k-1}\left(\exp \left(A_{i}\right)-\exp \left(-A_{i}\right)\right)^{1 / \alpha} \cdot\left(2 A_{k}\right)^{-1 / \alpha}\left(\exp \left(A_{k}\right)-\exp \left(-A_{k}\right)\right)^{1 / \alpha} \exp \left(\sum_{i=1}^{k} b^{i} \varepsilon s\right) \\
= & \prod_{i=1}^{k}\left(2 A_{i}\right)^{-1 / \alpha} \prod_{i=1}^{k}\left(\exp \left(A_{i}\right)-\exp \left(-A_{i}\right)\right)^{1 / \alpha} \cdot \exp \left(\sum_{i=1}^{k} b^{i} \varepsilon s\right),
\end{aligned}
$$

where each step holds by: homogeneity, the induction hypothesis, $\gamma_{s}=e^{s}$, and the definition of $N$. 
(29) allows us to conclude

$$
\begin{aligned}
\lambda_{g} & =\sup _{s \in S} \lim _{k \rightarrow \infty}\left(N_{s}^{k}\left(\prod_{q=1}^{k} \gamma_{s_{q}}^{\varepsilon}\right)\right)^{1 / k} \\
& =\sup _{s \in S} \lim _{k \rightarrow \infty}\left(\prod_{i=1}^{k}\left(2 A_{i}\right)^{-1 / \alpha} \prod_{i=1}^{k}\left(\exp \left(A_{i}\right)-\exp \left(-A_{i}\right)\right)^{1 / \alpha}\right)^{1 / k} \exp \left(\frac{1}{k} \sum_{i=1}^{k} b^{i} \varepsilon s\right) \\
& =\left(\frac{1-b}{2 \alpha \varepsilon r}\right)^{1 / \alpha}\left(\exp \left(\frac{\alpha \varepsilon r}{1-b}\right)-\exp \left(-\frac{\alpha \varepsilon r}{1-b}\right)\right)^{1 / \alpha}
\end{aligned}
$$

because $|b|<1$.

(29) also allows us to conclude that $(\forall s)\left(\forall \kappa_{5}, \kappa_{6}\right)$

$$
\begin{aligned}
&\left(\bar{u}_{s}\right)^{\alpha} \\
&= {\left[\kappa_{5}+\kappa_{6} \gamma_{s}^{\varepsilon} \sum_{r=0}^{\infty} d^{r} N_{s}^{r}\left(\prod_{q=1}^{r} \gamma_{s_{q}}^{\varepsilon}\right)\right]^{\alpha} } \\
&= {\left[\kappa_{5}+\kappa_{6} \gamma_{s}^{\varepsilon}\left(1+\sum_{r=1}^{\infty} d^{r} N_{s}^{r}\left(\prod_{q=1}^{r} \gamma_{s_{q}}^{\varepsilon}\right)\right)\right]^{\alpha} } \\
&= {\left[\kappa_{5}+\kappa_{6} \gamma_{s}^{\varepsilon}\left(1+\sum_{r=1}^{\infty} d^{r} \prod_{i=1}^{r}\left(2 A_{i}\right)^{-1 / \alpha} \prod_{i=1}^{r}\left(\exp \left(A_{i}\right)-\exp \left(-A_{i}\right)\right)^{1 / \alpha} \cdot \exp \left(\sum_{i=1}^{r} b^{i} \varepsilon s\right)\right)\right]^{\alpha} } \\
& \leq {\left[\kappa_{5}+\kappa_{6} \gamma_{s}^{\varepsilon}\left(1+\sum_{r=1}^{\infty} d^{r} \prod_{i=1}^{r}\left(2 A_{i}\right)^{-1 / \alpha} \prod_{i=1}^{r}\left(\exp \left(A_{i}\right)-\exp \left(-A_{i}\right)\right)^{1 / \alpha} \cdot\left(1+\exp \left(\frac{2 b \varepsilon s}{1-b}\right)\right)\right)\right]^{\alpha} } \\
&= {\left[\kappa_{5}+\kappa_{6} \gamma_{s}^{\varepsilon}\left(1+\left(1+\exp \left(\frac{2 b \varepsilon s}{1-b}\right)\right) \sum_{r=1}^{\infty} d^{r} \prod_{i=1}^{r}\left(2 A_{i}\right)^{-1 / \alpha} \prod_{i=1}^{r}\left(\exp \left(A_{i}\right)-\exp \left(-A_{i}\right)\right)^{1 / \alpha}\right)\right]^{\alpha} } \\
&= {\left[\kappa_{5}+\kappa_{6} \gamma_{s}^{\varepsilon}\left(1+\left(1+\exp \left(\frac{2 b \varepsilon s}{1-b}\right)\right) \sum_{r=1}^{\infty}\left[d\left(\prod_{i=1}^{r}\left(2 A_{i}\right)^{-1 / \alpha} \prod_{i=1}^{r}\left(\exp \left(A_{i}\right)-\exp \left(-A_{i}\right)\right)^{1 / \alpha}\right)^{1 / r}\right]^{r}\right)\right]^{\alpha} } \\
&= {\left[\kappa_{5}+\kappa_{6} \gamma_{s}^{\varepsilon}\left(1+\left(1+\exp \left(\frac{2 b \varepsilon s}{1-b}\right)\right) \kappa_{15}\right)\right]^{\alpha} } \\
&= {\left[\kappa_{5}+\kappa_{16} \gamma_{s}^{\varepsilon}+\kappa_{17} \exp \left(\frac{2 b \varepsilon s}{1-b}\right) \gamma_{s}^{\varepsilon}\right]^{\alpha} } \\
&= {\left[\kappa_{5}+\kappa_{16} \exp (\varepsilon s)+\kappa_{17} \exp \left(\frac{2 b \varepsilon s}{1-b}\right) \exp (\varepsilon s)\right]^{\alpha} } \\
&= {\left[\kappa_{5}+\kappa_{16} \exp (\varepsilon s)+\kappa_{17} \exp \left(\frac{1+b}{1-b} \varepsilon s\right)\right]^{\alpha} } \\
& \leq\left(3 \kappa_{5}\right)^{\alpha}+\left(3 \kappa_{16}\right)^{\alpha} \exp (\alpha \varepsilon s)+\left(3 \kappa_{17}\right)^{\alpha} \exp \left(\frac{1+b}{1-b} \alpha \varepsilon s\right) \\
&=\kappa_{18}+\kappa_{19} \exp \left(\kappa_{20} s\right)+\kappa_{21} \exp \left(\kappa_{22} s\right),
\end{aligned}
$$

where every $\kappa$ is a nonnegative real. The third equality holds by (29), the next inequality is justified by (28), and $\kappa_{15}$ is finite because the limit (as $r \rightarrow \infty$ ) of the inside of the brackets is less than 1 since $d<\lambda_{g}^{-1}$. Finally, the second inequality holds because of the 3 's.

Lemma C.2 shows that $E_{s}$ is u.q.c. beneath the RHS of (30): $\mathrm{m} 1$ is obvious and $\mathrm{m} 2$ holds because

$$
\begin{aligned}
& E_{s}\left(\kappa_{18}+\kappa_{19} \exp \left(\kappa_{20} s^{\prime}\right)+\kappa_{21} \exp \left(\kappa_{22} s^{\prime}\right)\right) \\
= & \kappa_{18}+\frac{\kappa_{19}}{2 \kappa_{20} r}\left(\exp \left(\kappa_{20} r\right)-\exp \left(-\kappa_{20} r\right)\right) \exp \left(b \kappa_{20} s\right)+\frac{\kappa_{21}}{2 \kappa_{22} r}\left(\exp \left(\kappa_{22} r\right)-\exp \left(-\kappa_{22} r\right)\right) \exp \left(b \kappa_{22} s\right) .
\end{aligned}
$$

Hence (30) shows that $E_{s}$ is u.q.c. beneath $\bar{u}^{\alpha}$. Hence $\left(E_{s}(\cdot)^{\alpha}\right)^{1 / \alpha}$ is u.q.c. beneath $\bar{u}$ by the monotonic invariance of u.q.c. (see U8 within Proof of Lemma B.1). 
Technology 3 (Gamma): This paragraph shows by induction on $k$ that

$$
(\forall s)(\forall k \geq 1) \quad N_{s}^{k}\left(\prod_{q=1}^{k} \gamma_{s_{q}}^{\varepsilon}\right)=\left(\frac{\prod_{i=0}^{k-1} \Gamma\left(\sum_{j=0}^{i} b^{j} \alpha \varepsilon+r\right)}{(\Gamma(r))^{k}}\right)^{1 / \alpha} \exp \left(\sum_{i=1}^{k} b^{i} \varepsilon \ln s\right),
$$

where $\Gamma$ is the gamma function. Note that

$$
\begin{aligned}
(\forall p>-r) & E_{s}\left(s^{\prime}\right)^{p} \\
& =\int_{0}^{\infty}\left(s^{\prime}\right)^{p} \frac{\left(s^{-b}\right)^{r}}{\Gamma(r)}\left(s^{\prime}\right)^{r-1} \exp \left(-s^{-b} s^{\prime}\right) d s^{\prime} \\
& =\frac{\left(s^{-b}\right)^{r}}{\Gamma(r)} \int_{0}^{\infty}\left(s^{\prime}\right)^{p+r-1} \exp \left(-s^{-b} s^{\prime}\right) d s^{\prime} \\
& =\frac{\left(s^{-b}\right)^{r}}{\Gamma(r)} \frac{\Gamma(p+r)}{\left(s^{-b}\right)^{p+r}} \\
& =\frac{\Gamma(p+r)}{\Gamma(r)} s^{b p}
\end{aligned}
$$

where the first equality is by Mood, Graybill, and Boes (1974, p. 540). The initial step $(k=1)$ is immediate by (32):

$$
\begin{aligned}
N_{s}\left(\gamma_{s_{1}}^{\varepsilon}\right) & =\left(E_{s} s_{1}^{\alpha \varepsilon}\right)^{1 / \alpha} \\
& =\left(\frac{\Gamma(\alpha \varepsilon+r)}{\Gamma(r)}\right)^{1 / \alpha} s^{b \varepsilon} .
\end{aligned}
$$

The following steps $(k \geq 2)$ hold as follows:

$$
\begin{aligned}
& N_{s}^{k}\left(\prod_{q=1}^{k} \gamma_{s_{q}}^{\varepsilon}\right) \\
= & N_{s}\left(\gamma_{s_{1}}^{\varepsilon} N_{s_{1}}^{k-1}\left(\prod_{q=2}^{k} \gamma_{s_{q}}^{\varepsilon}\right)\right) \\
= & N_{s}\left(\gamma_{s_{1}}^{\varepsilon}\left(\frac{\prod_{i=0}^{k-2} \Gamma\left(\sum_{j=0}^{i} b^{j} \alpha \varepsilon+r\right)}{(\Gamma(r))^{k-1}}\right)^{1 / \alpha} \exp \left(\sum_{i=1}^{k-1} b^{i} \varepsilon \ln s_{1}\right)\right) \\
= & N_{s}\left(\left(\frac{\prod_{i=0}^{k-2} \Gamma\left(\sum_{j=0}^{i} b^{j} \alpha \varepsilon+r\right)}{(\Gamma(r))^{k-1}}\right)^{1 / \alpha} \exp \left(\sum_{i=0}^{k-1} b^{i} \varepsilon \ln s_{1}\right)\right) \\
= & \left(\frac{\prod_{i=0}^{k-2} \Gamma\left(\sum_{j=0}^{i} b^{j} \alpha \varepsilon+r\right)}{(\Gamma(r))^{k-1}}\right)^{1 / \alpha}\left(E_{s} \exp \left(\sum_{i=0}^{k-1} b^{i} \alpha \varepsilon \ln s_{1}\right)\right)^{1 / \alpha} \\
= & \left(\frac{\prod_{i=0}^{k-2} \Gamma\left(\sum_{j=0}^{i} b^{j} \alpha \varepsilon+r\right)}{(\Gamma(r))^{k-1}}\right)^{1 / \alpha}\left(\frac{\Gamma\left(\sum_{i=0}^{k-1} b^{i} \alpha \varepsilon+r\right)}{\Gamma(r)}\right)^{1 / \alpha} \exp \left(b \sum_{i=0}^{k-1} b^{i} \varepsilon \ln s\right) \\
= & \left(\frac{\prod_{i=0}^{k-1} \Gamma\left(\sum_{j=0}^{i} b^{j} \alpha \varepsilon+r\right)}{(\Gamma(r))^{k}}\right)^{1 / \alpha} \exp \left(\sum_{i=1}^{k} b^{i} \varepsilon \ln s\right)
\end{aligned}
$$


where first five steps hold by: homogeneity, the induction hypothesis, $\gamma_{s}=s$, the definition of $N$, and (32).

(31) allows us to conclude

$$
\begin{aligned}
\lambda_{g} & =\sup _{s \in S} \lim _{k \rightarrow \infty}\left(N_{s}^{k}\left(\prod_{q=1}^{k} \gamma_{s_{q}}^{\varepsilon}\right)\right)^{1 / k} \\
& =\sup _{s \in S} \lim _{k \rightarrow \infty}\left(\left(\frac{\prod_{i=0}^{k-1} \Gamma\left(\sum_{j=0}^{i} b^{j} \alpha \varepsilon+r\right)}{(\Gamma(r))^{k}}\right)^{1 / \alpha} \exp \left(\sum_{i=1}^{k} b^{i} \varepsilon \ln s\right)\right)^{1 / k} \\
& =\sup _{s \in S} \lim _{k \rightarrow \infty}\left(\frac{\left(\prod_{i=0}^{k-1} \Gamma\left(\sum_{j=0}^{i} b^{j} \alpha \varepsilon+r\right)\right)^{1 / k}}{\Gamma(r)}\right)^{1 / \alpha} \exp \left(\frac{1}{k} \sum_{i=1}^{k} b^{i} \varepsilon \ln s\right) \\
& =\left(\frac{\Gamma(\alpha \varepsilon /(1-b)+r)}{\Gamma(r)}\right)^{1 / \alpha}
\end{aligned}
$$

because $|b|<1$.

(31) also allows us to conclude $(\forall s)$

$$
\begin{aligned}
& \left(\bar{u}_{s}\right)^{\alpha} \\
& =\left[\kappa_{5}+\kappa_{6} \gamma_{s}^{\varepsilon} \sum_{r=0}^{\infty} d^{r} N_{s}^{r}\left(\prod_{q=1}^{r} \gamma_{s_{q}}^{\varepsilon}\right)\right]^{\alpha} \\
& =\left[\kappa_{5}+\kappa_{6} \gamma_{s}^{\varepsilon}\left(1+\sum_{r=1}^{\infty} d^{r} N_{s}^{r}\left(\prod_{q=1}^{r} \gamma_{s_{q}}^{\varepsilon}\right)\right)\right]^{\alpha} \\
& =\left[\kappa_{5}+\kappa_{6} \gamma_{s}^{\varepsilon}\left(1+\sum_{r=1}^{\infty} d^{r}\left(\frac{\prod_{i=0}^{r-1} \Gamma\left(\sum_{j=0}^{i} b^{j} \alpha \varepsilon+r\right)}{(\Gamma(r))^{r}}\right)^{1 / \alpha} \exp \left(\sum_{i=1}^{r} b^{i} \varepsilon \ln s\right)\right)\right]^{\alpha} \\
& \leq\left[\kappa_{5}+\kappa_{6} \gamma_{s}^{\varepsilon}\left(1+\sum_{r=1}^{\infty} d^{r}\left(\frac{\prod_{i=0}^{r-1} \Gamma\left(\sum_{j=0}^{i} b^{j} \alpha \varepsilon+r\right)}{(\Gamma(r))^{r}}\right)^{1 / \alpha}\left(1+\exp \left(\frac{2 b \varepsilon \ln s}{1-b}\right)\right)\right)\right]^{\alpha} \\
& =\left[\kappa_{5}+\kappa_{6} \gamma_{s}^{\varepsilon}\left(1+\left(1+\exp \left(\frac{2 b \varepsilon \ln s}{1-b}\right)\right) \sum_{r=1}^{\infty} d^{r}\left(\frac{\prod_{i=0}^{r-1} \Gamma\left(\sum_{j=0}^{i} b^{j} \alpha \varepsilon+r\right)}{(\Gamma(r))^{r}}\right)^{1 / \alpha}\right)\right]^{\alpha} \\
& =\left[\kappa_{5}+\kappa_{6} \gamma_{s}^{\varepsilon}\left(1+\left(1+\exp \left(\frac{2 b \varepsilon \ln s}{1-b}\right)\right) \sum_{r=1}^{\infty}\left[d\left(\frac{\left(\prod_{i=0}^{r-1} \Gamma\left(\sum_{j=0}^{i} b^{j} \alpha \varepsilon+r\right)\right)^{1 / r}}{\Gamma(r)}\right)^{1 / \alpha}\right]^{r}\right)\right]^{\alpha} \\
& =\left[\kappa_{5}+\kappa_{6} \gamma_{s}^{\varepsilon}\left(1+\left(1+\exp \left(\frac{2 b \varepsilon \ln s}{1-b}\right)\right) \kappa_{23}\right)\right]^{\alpha} \\
& =\left[\kappa_{5}+\kappa_{24} \gamma_{s}^{\varepsilon}+\kappa_{25} \exp \left(\frac{2 b \varepsilon \ln s}{1-b}\right) \gamma_{s}^{\varepsilon}\right]^{\alpha} \\
& =\left[\kappa_{5}+\kappa_{24} s^{\varepsilon}+\kappa_{25} \exp \left(\frac{2 b \varepsilon \ln s}{1-b}\right) s^{\varepsilon}\right]^{\alpha} \\
& =\left[\kappa_{5}+\kappa_{24} s^{\varepsilon}+\kappa_{25} \exp \left(\frac{1+b}{1-b} \varepsilon \ln s\right)\right]^{\alpha} \\
& \leq\left(3 \kappa_{5}\right)^{\alpha}+\left(3 \kappa_{24}\right)^{\alpha} s^{\alpha \varepsilon}+\left(3 \kappa_{25}\right)^{\alpha} \exp \left(\frac{1+b}{1-b} \alpha \varepsilon \ln s\right)
\end{aligned}
$$




$$
=\kappa_{26}+\kappa_{27} s^{\kappa_{28}}+\kappa_{29} s^{\kappa_{30}},
$$

where every $k$ is a nonnegative real. The third equality holds by (31), the next inequality is justified by (28), and $\kappa_{23}$ is finite because the limit (as $r \rightarrow \infty$ ) of the inside of the brackets is less than 1 since $d<\lambda_{g}^{-1}$. Finally, the second inequality holds because of the 3 's.

Lemma C.2 shows that $E_{s}$ is u.q.c. beneath the RHS of (33): $\mathrm{m} 1$ is obvious and $\mathrm{m} 2$ holds because

$$
\begin{aligned}
& \int_{S}\left(\kappa_{26}+\kappa_{27}\left(s^{\prime}\right)^{\kappa_{28}}+\kappa_{29}\left(s^{\prime}\right)^{\kappa_{30}}\right) Q_{s}\left(d s^{\prime}\right) \\
= & \kappa_{26}+\kappa_{27} s^{b \kappa_{28}} \frac{\Gamma\left(\kappa_{28}+r\right)}{\Gamma(r)}+\kappa_{29} s^{b \kappa_{30}} \frac{\Gamma\left(\kappa_{30}+r\right)}{\Gamma(r)}
\end{aligned}
$$

by (32). Hence (33) shows that $E_{s}$ is u.q.c. beneath $\bar{u}^{\alpha}$. Hence $\left(E_{s}(\cdot)^{\alpha}\right)^{1 / \alpha}$ is u.q.c. beneath $\bar{u}$ by the monotonic invariance of u.q.c. (see U8 in Proof of Lemma B.1).

Proof D.4 (for Proposition 3): F1-F2 and U9 are obvious. U10 holds as well: $E$ is l.q.c. above 0 by Lemma C.4, and hence $M=\left(E(\cdot)^{a}\right)^{1 / a}$ is l.q.c. above $0^{1 / a}=0$ by the monotonic invariance of l.q.c. (see U10 in Proof of Lemma B.1). The remaining assumptions of Theorems B and C are identical to the conclusions of Theorem $D$.

$a \geq 1$. Here we show that $W$ and $M$ satisfy the Theorem D's assumptions. F1 and A1-A4 are obvious, A5 follows by the monotone convergence theorem, and A6 follows from Bertsekas and Shreve (1978, p. 144). Let $\varepsilon=e, \zeta_{s}=0$, and $\gamma_{s}=F_{s}^{\prime}(0)$; note that both $\bar{\delta}$ and $\delta$ equal $d$; and let $N$ equal $M$. Since $N=M$, N1-N2-N3 follow from A4-A5-A6 and N4 is immediate. N5 follows from the assumption $a \geq 1$, and N6 holds with equality. N7 holds by $\lambda_{1}=1$ and the assumption $d<1$. N8 and N9 hold by Lemma D.3(a) (applied at $\varepsilon=e$ and $\alpha=a$ ) and the proposition's inequality. N10 and $\mathrm{N} 11$ are vacuous because $\zeta_{s}=0$. Finally, N12 holds by Lemma D.3(b) (applied at $\kappa_{5}=k_{1}$ and $\kappa_{6}=k_{2} y_{-}^{\varepsilon}$ ).

$a \in(0,1)$. Consider the transformation $\phi(\cdot)=(\cdot)^{a}$ and the resulting aggregators $\widehat{W}(c, \hat{m})=$ $\left(c^{e}+d \hat{m}^{1 / a}\right)^{a}$ and $\widehat{M}_{s} u=\int u d Q_{s}$. By Lemma B.1( $a$ and $\left.\mathrm{b}\right)$, it suffices to show that $\widehat{W}$ and $\widehat{M}$ satisfy Theorem D's assumptions. F1 and A1-A4 are obvious, A5 follows by the monotone convergence theorem, and A6 follows from Bertsekas and Shreve (1978, p. 144). Let $\zeta_{s}=0$ and $\gamma_{s}=F_{s}^{\prime}(0)$; let $\varepsilon=a e \in(0,1]$ since

$$
(\forall c) \quad \widehat{W}(c, 0)=(W(c, 0))^{a}=c^{a e} ;
$$

note that both $\bar{\delta}$ and $\delta$ equal $d^{a}$ since

$$
\sup _{c} \widehat{W}_{2}(c, \hat{m})=\sup _{c}\left(c^{e}+d \hat{m}^{1 / a}\right)^{a-1} d \hat{m}^{(1-a) / a}=d^{a}
$$

and let $N=\widehat{M}$. Since $N=\widehat{M}$, N1-N2-N3 follow from A4-A5-A6 and N4 is immediate. N5 and N6 hold with equality because $N$ is the expectation operator. N7 holds by $\lambda_{1}=1$ and the assumption $d<1$. N8 and N9 holds by Lemma D.3(a) (applied at $\varepsilon=a e$ and $\alpha=1$ ), the proposition's inequality, and $\delta=d^{a}$. N10 and N11 are vacuous since $\zeta_{3}=0$. Finally, N12 holds by Lemma D.3(b) (applied at $\kappa_{5}=k_{1}$ and $\kappa_{6}=k_{2} y_{-}^{\varepsilon}$ ). 
Proof D.5 (for Proposition 4): F1-F2 and U9 are obvious. U10 holds as well: $E$ is l.q.c. above 0 by Lemma C.4, and hence $M=\left(E(\cdot)^{a}\right)^{1 / a}$ is l.q.c. above $0^{1 / a}=0$ by the monotonic invariance of l.q.c. (see U10 in Proof of Lemma B.1). The remaining assumptions of Theorems B and C are identical to the conclusions of Theorem D.

$a \geq 1$. Here we show that $W$ and $M$ satisfy the Theorem D's assumptions. F1 and A1-A4 are obvious, A5 follows by the monotone convergence theorem, and A6 follows from Bertsekas and Shreve (1978, p. 144). Let $\varepsilon=e, \zeta_{s}=0$, and $\gamma_{s}=F_{s}^{\prime}(0)$; note that $\bar{\delta}=d$ and $\delta=0$ because $\sup _{c} W_{2}(c, m)=d(1+m)^{h-1}$; and let $N$ equal $M$. Since $N=M$, N1-N2-N3 follow from A4-A5-A6 and N4 is immediate. N5 follows from the assumption $a \geq 1$, and N6 holds with equality. N7 holds by $\lambda_{1}=1$ and the assumption $d<1$. N8 and N9 hold because $\delta=0$ and because $\lambda_{g}$ is finite by Lemma D.3(a) (applied at $\varepsilon=e$ and $\alpha=a$ ). N10 and N11 are vacuous since $\zeta_{s}=0$. Finally, N12 holds by Lemma D.3(b) (applied at $\kappa_{5}=k_{1}$ and $\kappa_{6}=k_{2} y_{-}^{\varepsilon}$ ).

$a \in(0,1)$. Consider the transformation $\phi(\cdot)=(\cdot)^{a}$ and the resulting aggregators $\widehat{M}_{s} u=\int u d Q$, and $\widehat{W}(c, \hat{m})=\left(c^{e}+D\left(\hat{m}^{1 / a}\right)\right)^{a}$, where $D(m) \equiv(d / h)\left((1+m)^{h}-1\right)$ if $h \neq 0$ and $D(m) \equiv d \ln (1+m)$ if $h=0$. By Lemma B.1(a and $\mathrm{b})$, it suffices to show that $\widehat{W}$ and $\widehat{M}$ satisfy Theorem D's assumptions. F1 and A1-A4 are obvious, A5 follows by the monotone convergence theorem, and A6 follows from Bertsekas and Shreve (1978, p. 144). Let $\zeta_{s}=0$ and $\gamma_{s}=F_{s}^{\prime}(0)$; and let $\varepsilon=a e \in(0,1]$ since

$$
\text { ( } \forall c) ~ \widehat{W}(c, 0)=(W(c, 0))^{a}=c^{a e} .
$$

Note that

$$
\begin{aligned}
(\forall \hat{m}) \quad & \sup _{c} \widehat{W}_{2}(c, \hat{m}) \\
= & \sup _{c}\left(c^{e}+D\left(\hat{m}^{1 / a}\right)\right)^{a-1} D^{\prime}\left(\hat{m}^{1 / a}\right) \hat{m}^{(1-a) / a} \\
= & \left(D\left(\hat{m}^{1 / a}\right)\right)^{a-1} D^{\prime}\left(\hat{m}^{1 / a}\right) \hat{m}^{(1-a) / a} \\
= & \left(D\left(\hat{m}^{1 / a}\right) / \hat{m}^{1 / a}\right)^{a-1} D^{\prime}\left(\hat{m}^{1 / a}\right) \\
\leq & \left(D^{\prime}\left(\hat{m}^{1 / a}\right)\right)^{a-1} D^{\prime}\left(\hat{m}^{1 / a}\right) \\
= & \left(D^{\prime}\left(\hat{m}^{1 / a}\right)\right)^{a} \\
= & d^{a}\left(\hat{m}^{1 / a}+1\right)^{a(h-1)}
\end{aligned}
$$

(the second equality holds since $a-1<0$ and the inequality holds since $D$ is concave and $D(0)=0)$. Hence $\delta=0$ and $\bar{\delta}=d^{a}\left(=\widehat{W}_{2}(0,0)\right)$. Finally, let $N=\widehat{M}$. Since $N=\widehat{M}$, N1-N2-N3 follow from A4-A5-A6 and $\mathrm{N} 4$ is immediate. $\mathrm{N} 6$ and $\mathrm{N} 5$ hold with equality because $N$ is the expectation operator. $\mathrm{N} 7$ holds by $\lambda_{1}=1$ and the assumption $d<1$. N8 and N9 hold because $\delta=0$ and because $\lambda_{g}$ is finite by Lemma D.3(a) (applied at $\varepsilon=a e$ and $\alpha=1$ ). N10 and N11 are vacuous since $\zeta_{s}=0$. Finally, N12 holds by Lemma D.3(b) (applied at $\kappa_{5}=k_{1}$ and $\kappa_{6}=k_{2} y_{-}^{\varepsilon}$ ). 


\section{Appendix E: Lemmas and Proofs for Section 4 (Nonpositive Preferences)}

Proof E.1 (for Theorem E): Well-Definition. Take any $s$ and $c . U_{s}(c)$ is defined as the limit of a sequence. Each element of the sequence is well-defined by B5. The limit of the sequence exists because the sequence is weakly decreasing (by B1 and B3) and contained in the closed set $\bar{\Re}$.

U1, U4 - U7. U1 (monotonicity) follows immediately from B1, B3 and the definition of $U$. U5, U6, and U7 are implied by B1, B2, and B3. U4 (Koopmans' equation) holds because

$$
\begin{aligned}
& (\forall s)(\forall y)(\forall c \leq 0 \bar{F}(y)) \\
& U_{s}(c) \\
= & \lim _{t \rightarrow \infty} W\left(c_{0}, M_{s} W\left(c_{1}, M \cdots W\left(c_{t}, 0\right) \cdots\right)\right) \\
= & W\left(c_{0}, \lim _{t \rightarrow \infty} M_{s} W\left(c_{1}, M \cdots W\left(c_{t}, 0\right) \cdots\right)\right) \\
= & W\left(c_{0}, M_{s} \lim _{t \rightarrow \infty} W\left(c_{1}, M \cdots W\left(c_{t}, 0\right) \cdots\right)\right) \\
= & W\left(c_{0}, M_{s} U\left({ }_{1} c\right)\right) .
\end{aligned}
$$

These four equalities hold by the definition of $U, \mathbf{B} 2, \mathrm{~B} 4$, and the definition of $U$ again.

U2 (Upper Convergence). Take any $s$ and $y$, and any $c$ such that $c \leq{ }_{0} \bar{F}(y)$. Note that

$$
\begin{aligned}
& U_{s}(c) \\
= & \lim _{t \rightarrow \infty} W\left(c_{0}, M_{s} W\left(c_{1}, M \cdots W\left(c_{t}, 0\right) \cdots\right)\right) \\
\geq & \lim _{t \rightarrow \infty} W\left(c_{0}, M_{s} W\left(c_{1}, M \cdots W\left(c_{t}, M \lim _{r \rightarrow \infty} W\left(\bar{F}^{t+1}(y), M \cdots W\left(\bar{F}^{r}(y), 0\right) \cdots\right)\right) \cdots\right)\right) \\
= & \left.\lim _{t \rightarrow \infty} W\left(c_{0}, M_{s} W\left(c_{1}, M \cdots W\left(c_{t}, M U(t+1) \bar{F}(y)\right)\right) \cdots\right)\right) \\
= & \lim _{t \rightarrow \infty} U_{s}\left(c_{t} c_{t+1} \bar{F}(y)\right) \\
\geq & U_{s}(c) .
\end{aligned}
$$

The first inequality holds by B1, B3, and because the range of $M$ is nonpositive. The second equality holds by the definition of $U$ applied at each of the period $t+1$ histories. The last two steps follow from Koopmans' equation and monotonicity, which have already been proven. The inequalities imply that all limits equal $U_{s}(c)$. In particular, $\lim _{t \rightarrow \infty} U_{s}\left(0 c_{t}, t+1 \bar{F}(y)\right)=U_{s}(c)$, as required.

Lemma E.2: Suppose $\eta>0$. (a) Then

$$
\begin{array}{lll}
(\forall s)(\forall y) & E_{s}(F(y))^{\eta}=y^{\eta} \exp \left(\eta b s+\frac{1}{2} \eta^{2} \sigma^{2}\right) & \text { in Technology 1, } \\
(\forall s)(\forall y) & E_{s}(F(y))^{\eta}=\frac{y^{\eta}}{2 \eta r}(\exp (\eta(b s+r))-\exp (\eta(b s-r))) & \text { in Technology 2, and } \\
(\forall s)(\forall y) & E_{s}(F(y))^{\eta}=y^{\eta} s^{\eta b} \frac{\Gamma(\eta+r)}{\Gamma(r)} & \text { in Technology 3. }
\end{array}
$$


(b) Furthermore, $(\forall y) E_{s}$ is u.q.c. beneath $(\bar{F}(y))^{\eta}$ in any of the three technologies.

Proof: (a) is proven with the algebraic identities (26) and (32) in Lemma D.3's proof. (b) holds by Lemma C.2: $\mathrm{m} 1$ holds because the distributions have densities which are continuous almost everywhere, and $\mathrm{m} 2$ holds by (a).

Lemma E.3: Suppose $W: \Re_{+} \times \bar{\Re}_{-} \rightarrow \bar{\Re}_{-}$is defined by

$$
W(c, m)=-c^{\varepsilon}+D(m),
$$

where $D: \bar{\Re}_{-} \rightarrow \bar{\Re}_{-}$is weakly increasing, is upper semi-continuous, and satisfies $D(0)=0$. Also suppose that $M$ is defined as in Utility Example 3. Then the assumptions of Theorem A are met by Technologies 1, 2 , and 3 .

Proof: Consider the assumptions of Theorem E. F1 and B1-B3 are obvious. B5 holds by Bertsekas and Shreve (1978, p. 144). B4 holds when $\alpha \neq 0$ because

$$
\begin{aligned}
& (\forall s)(\forall y)\left(\forall \text { weakly decreasing }\left\langle u^{n}\right\rangle_{n} \leq W(\bar{F}(y), 0)\right) \\
& \lim _{n \rightarrow \infty} M_{s} u^{n} \\
= & \lim _{n \rightarrow \infty}-\left[E_{s}\left(-u^{n}\right)^{\alpha}\right]^{1 / \alpha} \\
= & -\left[\lim _{n \rightarrow \infty} E_{s}\left(-u^{n}\right)^{\alpha}\right]^{1 / \alpha} \\
= & -\left[E_{s} \lim _{n \rightarrow \infty}\left(-u^{n}\right)^{\alpha}\right]^{1 / \alpha} \\
= & -\left[E_{s}\left(-\lim _{n \rightarrow \infty} u^{n}\right)^{\alpha}\right]^{1 / \alpha} \\
= & M_{s} \lim _{n \rightarrow \infty} u^{n} .
\end{aligned}
$$

The middle equality follows from the monotone convergence theorem: if $\alpha>0,\left\langle\left(-u^{n}\right)^{\alpha}\right\rangle$ is a weakly increasing nonnegative sequence and the restriction $\left\langle u^{n}\right\rangle_{n} \leq W(\bar{F}(y), 0)$ is superfluous; and if $\alpha<0$, $\left\langle\left(-u^{n}\right)^{\alpha}\right\rangle$ is a weakly decreasing nonnegative sequence which is bounded from above by

$$
(-W(\bar{F}(y), 0))^{\alpha}=(\bar{F}(y))^{\alpha \varepsilon}
$$

which is integrable in each of the three technologies by Lemma E.2(a) (set $\eta=\alpha \varepsilon$ ). B4 also holds when $\alpha=0$ because

$$
\begin{aligned}
& (\forall s)(\forall y)\left(\forall \text { weakly decreasing }\left\langle u^{n}\right\rangle_{n} \leq W(\bar{F}(y), 0)\right) \\
& \lim _{n \rightarrow \infty} M_{s} u^{n} \\
= & \lim _{n \rightarrow \infty}-\exp \left[E_{s} \ln \left(-u^{n}\right)\right] \\
= & -\exp \left[\lim _{n \rightarrow \infty} E_{s} \ln \left(-u^{n}\right)\right]
\end{aligned}
$$




$$
\begin{aligned}
& =-\exp \left[E_{s} \lim _{n \rightarrow \infty} \ln \left(-u^{n}\right)\right] \\
& =-\exp \left[E_{s} \ln \left(-\lim _{n \rightarrow \infty} u^{n}\right)\right] \\
& =M_{s} \lim _{n \rightarrow \infty} u^{n} .
\end{aligned}
$$

The middle equality follows from the monotonic convergence theorem: $\left\langle\ln \left(-u^{n}\right)\right\rangle$ is a weakly increasing sequence which is bounded from below by

$$
\ln (-W(\bar{F}(y), 0))=\varepsilon \ln (\bar{F}(y)) \geq \varepsilon \bar{F}(y)
$$

(recall $\varepsilon<0$ ) which is integrable at each $s$ in each of the three technologies by Lemma E.2(a) (set $\eta=1$ ).

Since F1 and B1-B5 are satisfied, Theorem E implies that U1, U4-U7 and upper convergence are satisfied. Only U8 remains. Lemma B.1(a) shows that for any trasformation $\phi,[U, W, M$,$] satisfies U8$ iff $[\widehat{U}, \widehat{W}, \widehat{M}$,$] satisfies U8. When \alpha>0$, let $\phi(u)=-(-u)^{\alpha}$ and note that $[\widehat{U}, \widehat{W}, \widehat{M}$,$] satisfies U8 iff$ $(\forall y) E=\phi M \phi^{-1}$ is u.q.c. beneath $\phi J^{+} \circ \bar{F}(y)$. Since $(\forall y) \phi J^{+} \circ \bar{F}(y) \leq 0$, this follows immediately from Lemma C.2 and the observation that M2 holds because $\bar{u}$ is bounded from above by a constant. When $\alpha=0$, let $\phi(u)=-\ln (-u)$ and note that $[\widehat{U}, \widehat{W}, \widehat{M}$,$] satisfies \mathrm{U} 8$ iff $(\forall y) E=\phi M \phi^{-1}$ is u.q.c. beneath $\phi J^{+} \circ \bar{F}(y)$. This holds if $(\forall y) E$ is u.q.c. beneath $-\varepsilon \bar{F}(y)$ because

$$
\begin{aligned}
(\forall y) \quad & \phi J^{+} \circ \bar{F}(y) \\
\leq & \phi\left(-(\bar{F}(y))^{\varepsilon}\right) \\
= & -\ln (\bar{F}(y))^{\varepsilon} \\
\leq & -\varepsilon \bar{F}(y) .
\end{aligned}
$$

This holds by Lemma C.2 and Lemma E.2(b) (set $\eta=1$ ). When $\alpha<0$, let $\phi(u)=(-u)^{\alpha}$ and note that $[\widehat{U}, \widehat{W}, \widehat{M}$,$] satisfies \mathrm{U} 8$ iff $(\forall y) E=\phi M \phi^{-1}$ is u.q.c. beneath $\phi J^{+} \circ \bar{F}(y)$. This holds if $(\forall y) E$ is u.q.c. beneath $(\bar{F}(y))^{\alpha \varepsilon}$ because

$$
\begin{aligned}
& \phi J^{+} \circ \bar{F}(y) \\
\leq & \phi\left(-(\bar{F}(y))^{\varepsilon}\right) \\
= & (\bar{F}(y))^{\alpha \varepsilon} .
\end{aligned}
$$

This holds by Lemma C.2 and Lemma E.2(b) (set $\eta=\alpha \varepsilon>0$ ). 


\section{REFERENCES}

Benhabib, Jess, Saqib Jafarey, and Kazuo Nishimura (1988): “The Dynamics of Efficient Intertemporal Allocations with Many Agents, Recursive Preferences, and Production", Journal of Economic Theory 44, 301-320.

Berge, Claude (1963): Topological Spaces, Oliver and Boyd, Edinburgh.

Bertsekas, Dimitri P. and Steven E. Shreve (1978): Stochastic Optimal Control: The Discrete Case, Academic Press, New York.

Bhattacharya, Rabi N. and Mukul Majumdar (1989): "Controlled Semi-Markov Models - The Discounted Case", Journal of Statistical Planning and Inference 21, 365-381.

York.

Billingsley, Patrick (1986): Probability and Measure (Second Edition), John Wiley and Sons, New $36,226-235$.

Blackwell, David (1965): "Discounted Dynamic Programming", Annals of Mathematical Statistics

Boyd, John H., III (1990): "Recursive Utility and the Ramsey Problem", Journal of Economic Theory 50, 326-345.

Brock, W. A. and David Gale (1969): "Optimal Growth under Factor Augmenting Progress", Journal of Economic Theory 1, 229-243.

Epstein, Larry G. (1983): "Stationary Cardinal Utility and Optimal Growth Under Uncertainty", Journal of Economic Theory 31, 133-152.

Epstein, Larry G. and Tan Wang (1994a): "Intertemporal Asset Pricing under Knightian Uncertainty", Econometrica 62, 283-323.

Epstein, Larry G. and Tan Wang (1994b): "Uncertainty, Risk-Neutral Measures and Security Price Booms and Crashes", University of Toronto, mimeo.

Epstein, Larry G. and Stanley E. Zin (1989): "Substitution, Risk Aversion, and the Temporal Behavior of Consumption and Asset Returns: A Theoretical Framework", Econometrica 57, 937-970. 43-60.

Farmer, Roger E. A. (1990): "RINCE Preferences", Quarterly Journal of Economics, February,

Kocherlakota, Narayana R. (1990): “On the 'Discount' Factor in Growth Economies”, Journal of Monetary Economics 25, 43-47. 309.

Koopmans, Tjalling C. (1960): “Stationary Ordinal Utility and Impatience", Econometrica 28, 287-

Koopmans, Tjalling C., Peter A. Diamond, and Robert E. Williamson (1964): "Stationary Utility and Time Perspective", Econometrica 32, 82-100.

Kreps, David M. and Evan L. Porteus (1978): "Temporal Resolution of Uncertainty and Dynamic Choice Theory", Econometrica 46, 185-200. 
Lucas, Robert E. Jr. and Nancy L. Stokey (1984): "Optimal Growth with Many Consumers", Journal of Economic Theory 32, 139-171. $30 \mathrm{~A}, 211-216$.

Maitra, Ashok (1968): “Discounted Dynamic Programming on Compact Metric Spaces", Sankhya

Mehra, Rajnish and Edward C. Prescott (1985): "The Equity Premium: A Puzzle", Journal of Monetary Economics 15, 145-161.

Mood, Alexander M., Franklin A. Graybill, and Duane C. Boes (1974): Introduction to the Theory of Statistics (Third Edition), MaGraw-Hill.

Ozaki, Hiroyuki and Peter A. Streufert (1992): "Nonlinear Dynamic Programming for Nonlinear Stochastic Objectives", University of Wisconsin-Madison, SSRI Workshop Series No. 9228 (similar to Section 2 of this paper).

Ozaki, Hiroyuki and Peter A. Streufert (1993): "An Application of Capacity Theory to Nonlinear Dynamic Programming", University of Wisconsin-Madison, January, mimeo.

Schäl, M. (1978): "An Operator-Theoretical Treatment of Negative Dynamic Programming", in Dynamic Programming and Its Applications, Martin L. Putermann (ed.), Academic Press.

Stokey, Nancy L. and Robert E. Lucas, Jr. (1989): Recursive Methods in Economic Dynamics, Harvard University Press, Cambridge, MA. 871-890.

Strauch, R. E. (1966): “Negative Dynamic Programming”, Annals of Mathematical Statistics 37,

Streufert, Peter A. (1990): "Stationary Recursive Utility and Dynamic Programming under the Assumption of Biconvergence", Review of Economic Studies 57, 79-97.

Streufert, Peter A. (1991): "Nonnegative Stochastic Dynamic Preferences", Stanford Institute for Theoretical Economics, Technical Report No. 22.

Streufert, Peter A. (1992): “An Abstract Topological Approach to Dynamic Programming", Journal of Mathematical Economics 21, 59-88.

Uzawa, Hirofumi (1968): "Time Preference, the Consumption Function and Optimum Asset Holdings", in Value, Capital and Growth: Papers in Honour of Sir John Hicks, Chicago: Aldine.

Wagner, Daniel H. (1977): "Survey of Measurable Selection Theorems", SIAM Journal of Control and Optimization 15, 859-903.

Weil, Philippe (1990): “Nonexpected Utility in Macroeconomics", Quarterly Journal of Economics February, 29-42. 